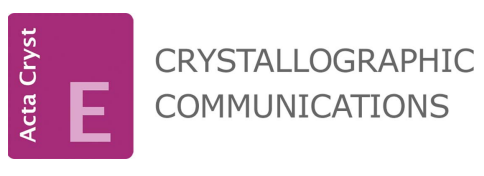

ISSN 2056-9890

\section{An investigation to elucidate the factors dictating the crystal structure of seven ammonium carboxylate molecular salts}

\author{
Jacques Blignaut and Andreas Lemmerer*
}

Received 27 October 2017

Accepted 13 December 2017

Edited by C. Massera, Università di Parma, Italy

Keywords: crystal structure; ammonium carboxylate salts; graph set; hydrogen bonding.

CCDC references: $1811019 ; 1811018$; 1811017; 1811016; 1811015; 1811014; 1811013

Supporting information: this article has supporting information at journals.iucr.org/e

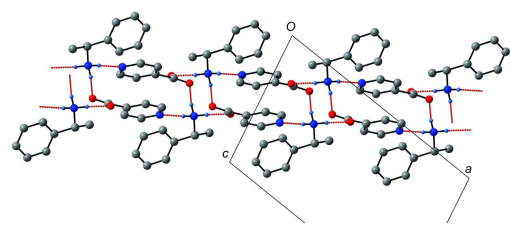

OPEN $\odot$ ACCESS
Molecular Sciences Institute, School of Chemistry, University of the Witwatersrand, Private Bag, PO WITS, 2050, Johannesburg, South Africa. *Correspondence e-mail: andreas.lemmerer@wits.ac.za

The crystal structures of seven ammonium carboxylate salts are reported, namely $(R S)$-1-phenylethan-1-aminium isonicotinate, $\mathrm{C}_{8} \mathrm{H}_{12} \mathrm{~N}^{+} \cdot \mathrm{C}_{6} \mathrm{H}_{4} \mathrm{~N}_{1} \mathrm{O}_{2}{ }^{-}$, (I), $(R S)$-1-phenylethan-1-aminium flurbiprofenate [or 2-(3-fluoro-4-phenylphenyl)propanoate], $\mathrm{C}_{8} \mathrm{H}_{12} \mathrm{~N}^{+} \cdot \mathrm{C}_{15} \mathrm{H}_{12} \mathrm{FO}_{2}{ }^{-}$, (II), (RS)-1-phenylethan-1-aminium 2-chloro-4-nitrobenzoate, $\mathrm{C}_{8} \mathrm{H}_{12} \mathrm{~N}^{+} \cdot \mathrm{C}_{7} \mathrm{H}_{3} \mathrm{ClNO}_{4}{ }^{-}$, (III), (RS)-1-phenylethan-1aminium 4-iodobenzoate, $\mathrm{C}_{8} \mathrm{H}_{12} \mathrm{~N}^{+} \cdot \mathrm{C}_{7} \mathrm{H}_{4} \mathrm{IO}_{2}{ }^{-}$, (IV), (S)-1-cyclohexylethan-1aminium 2-chloro-4-nitrobenzoate, $\mathrm{C}_{8} \mathrm{H}_{18} \mathrm{~N}^{+} \cdot \mathrm{C}_{7} \mathrm{H}_{3} \mathrm{ClNO}_{4}{ }^{-}$, (V), 2-(cyclohex-1en-1-yl)ethan-1-aminium 4-bromobenzoate, $\mathrm{C}_{8} \mathrm{H}_{16} \mathrm{~N}^{+} \cdot \mathrm{C}_{7} \mathrm{H}_{4} \mathrm{BrO}_{2}{ }^{-}$, (VI), and (S)-1-cyclohexylethan-1-aminium 4-bromobenzoate, $\mathrm{C}_{8} \mathrm{H}_{18} \mathrm{~N}^{+} \cdot \mathrm{C}_{7} \mathrm{H}_{4} \mathrm{BrO}_{2}{ }^{-}$, (VII). Salts (II) to (VII) feature three $\mathrm{N}^{+}-\mathrm{H} \cdots \mathrm{O}^{-}$hydrogen bonds, which form one-dimensional hydrogen-bonded ladders. Salts (II), (III), (IV), (V) and (VII) have a type II ladder system despite the presence of halogen bonding and other intermolecular interactions, whereas (VI) has a type III ladder system. Salt (I) has a unique hydrogen-bonded system of ladders, featuring both $\mathrm{N}^{+}-$ $\mathrm{H} \cdots \mathrm{O}^{-}$and $\mathrm{N}^{+}-\mathrm{H} \cdots \mathrm{N}$ hydrogen bonds owing to the presence of the pyridine functional group. The presence of an additional hydrogen-bond acceptor on the carboxylate cation disrupts the formation of the ubiquitous type II and III ladder found predominately in ammonium carboxylate salts. Halogen bonding, however, has no influence on their formation.

\section{Chemical context}

Crystal engineering, the conception and synthesis of molecular solid-state structures, is fundamentally based upon the discernment and subsequent exploitation of intermolecular interactions. Thus, primarily non-covalent bonding is used to achieve the organization of molecules and ions in the solid state in order to produce materials with desired properties. Examples of such materials include organic field-effect transistors, hole collectors in organic photovoltaic cells (Snaith, 2013), laser materials (Tessler, 1999) as well as organic light-emitting diodes and semiconductors (Odom et al., 2003). The two principle forces exploited in the design of molecular solids are hydrogen bonding and coordination complexation (Desiraju, 1989).

This work will focus on the effects of hydrogen bonding. In particular, we have investigated the effects thereof of changing both the structure and stereochemistry of the constituents on the robust ionic supramolecular heterosynthons generated by ammonium carboxylate salts $\left(R-\mathrm{NH}_{3}{ }^{+}\right) \cdot\left(R-\mathrm{COO}^{-}\right)$, where $R$ often contains a phenylethyl group generating chiral molecules (Kinbara et al., 1996). It is known from a wide variety of structural studies that ammonium carboxylate salts predom- 
inately form two types of hydrogen-bonded one-dimensional ladders in the solid state (Odendal et al., 2010). These are classified as type II and type III, where type II consists of repeating hydrogen-bonded rings with the descriptor $R_{4}^{3}(10)$ (Bernstein et al., 1995), while type III consists of alternating $R_{2}^{2}(8)$ and $R_{4}^{4}(12)$ rings. The robustness and perturbation of these ladders as a function of the structure and stereochemistry of the constituent ions have been tested via the crystallization of a variety of ammonium carboxylate salts. The seven salts reported here are (see Scheme): (RS)-1-phenylethan-1-aminium isonicotinate, (I), $(R S)$-1-phenylethan-1aminium flurbiprofenate, (II), $(R S)$-1-phenylethan-1-aminium 2-chloro-4-nitro-benzoate, (III), (RS)-1-phenylethan-1aminium 4-iodobenzoate, (IV), (S)-1-cyclohexylethan-1aminium 2-chloro-4-nitro-benzoate, (V), 2-(cyclohex-1-en-1yl)ethan-1-aminium 4-bromobenzoate, (VI), and (S)-1-cyclohexylethan-1-aminium 4-bromobenzoate, (VII).<smiles>C[C@H](N)c1ccccc1CSc1ccccc1</smiles>

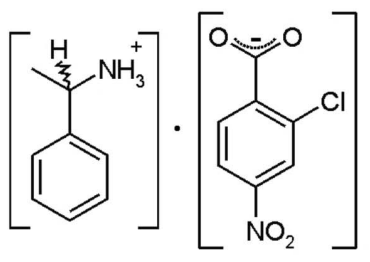

(III)

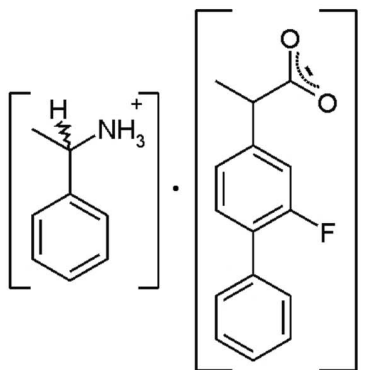

(II)

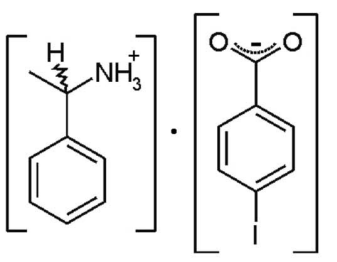

(IV)<smiles>C[C@H](N)C1CCCCC1CSc1ccc([N+](=O)[O-])cc1C(=O)O</smiles>

(V)

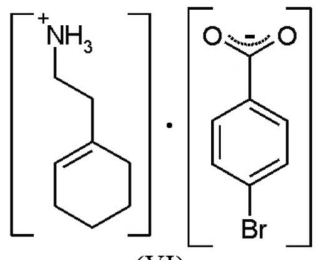

(VI)
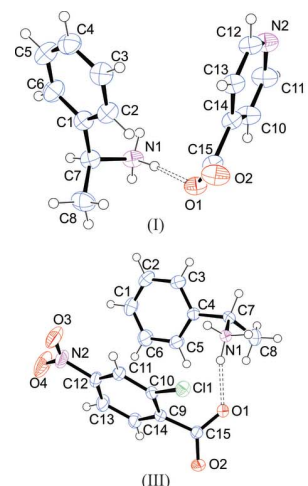

(III)
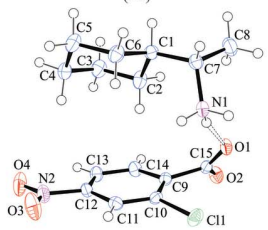

(V)

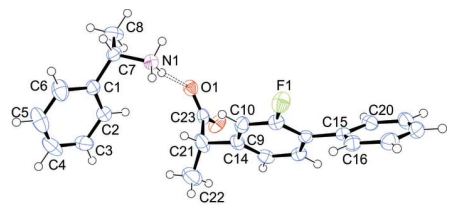

(II)

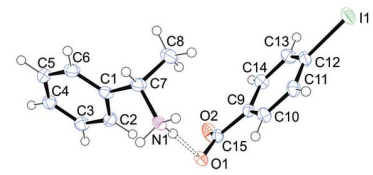

(IV)
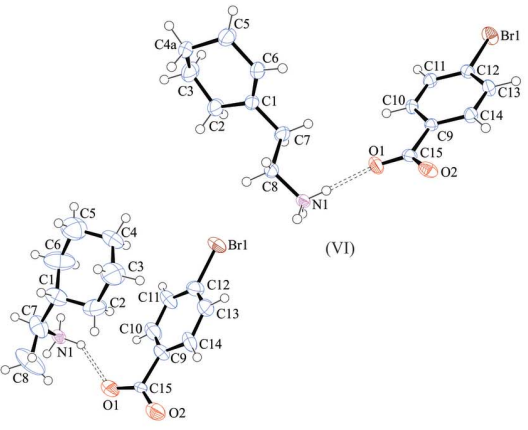

(VII)
Figure 1

Perspective views of compounds (I)-(VII), showing the atom-numbering schemes. Displacement ellipsoids are drawn at the $50 \%$ probability level and $\mathrm{H}$ atoms are shown as small spheres of arbitrary radii. The dashed lines indicate the symmetry-independent $\mathrm{N}^{+}-\mathrm{H} \cdots \mathrm{O}^{-}$or $\mathrm{N}^{+}-\mathrm{H} \cdots \mathrm{N}$ hydrogen bonds.

in $\mathrm{p} K_{\mathrm{a}}$ values depicted in Table $\mathrm{S} 1$ in the supporting information, all the compounds considered in this work should be in the form of salts and hence possess charge-assisted hydrogen bonds, which are considered to be a stronger and more robust supramolecular synthon than the same between neutral molecules (Lemmerer et al., 2008a). All structures crystallize with a 1:1 ratio of ammonium cation to benzoate anion, with all molecules on general positions. The asymmetric units and atom-numbering schemes are shown in Fig. 1.

\section{Supramolecular features}

Salt (I) consists of one 1-phenylethan-1-aminium cation and one isonicotinate anion. The ammonium group forms three charge-assisted hydrogen bonds, shown in Fig. $2 a$. The first of these bonds involves the $\mathrm{O} 2$ atom of the isonicotinate anion (i) (see Table 1) and is designated $a$. The second involves the $\mathrm{O} 1$ atom of the isonicotinate anion in the asymmetric unit and is designated $b$. The third involves the pyridine ring nitrogen of a third isonicotinate anion (ii) and is designated $c$. The $b$ and $c$ hydrogen bonds form a ring structure involving two of each kind of bond, consisting of two molecules of both 1-phenylethan-1-aminium and isonicotinate (See Fig. 2a). The graph set of this pattern is $R_{4}^{4}(18)$. A larger $\mathrm{R}_{8}^{8}(30)$ ring is formed using all three hydrogen bonds involving four of both 1-phenylethan-1-aminium and isonicotinate ions. Overall, this forms a 2-D sheet as shown in Fig. $2 b$. As neither of the two

\section{Structural commentary}

An amine and a carboxylic acid will combine to form a salt if the difference in $\mathrm{p} K_{\mathrm{a}}$ 's is approximately 3 or greater (Bhogala et al., 2005; Lemmerer et al., 2015). Thus, from the differences 
Table 1

Hydrogen-bond geometry $\left(\AA{ }^{\circ}\right)$ for $(\mathrm{I})$.

\begin{tabular}{lllll}
\hline$D-\mathrm{H} \cdots A$ & $D-\mathrm{H}$ & $\mathrm{H} \cdots A$ & $D \cdots A$ & $D-\mathrm{H} \cdots A$ \\
\hline $\mathrm{N} 1-\mathrm{H} 1 B \cdots \mathrm{O} 2^{\mathrm{i}}$ & $0.95(2)$ & $1.80(2)$ & $2.747(2)$ & $176(2)$ \\
$\mathrm{N} 1-\mathrm{H} 1 C \cdots \mathrm{O} 1$ & $0.98(2)$ & $1.81(2)$ & $2.783(2)$ & $174(2)$ \\
$\mathrm{N} 1-\mathrm{H} 1 A \cdots \mathrm{N} 2^{\mathrm{ii}}$ & $0.93(2)$ & $1.93(2)$ & $2.856(2)$ & $175(2)$ \\
\hline
\end{tabular}

Symmetry codes: (i) $-x+\frac{3}{2}, y-\frac{1}{2},-z+\frac{1}{2}$; (ii) $-x+1,-y+1,-z$.

expected type II or type III ladders are formed it seems that the additional hydrogen-bond acceptor in the form of the nitrogen atom of the pyridine ring disrupts their formation.

In salt (II), the asymmetric unit consists of one 1-phenylethan-1-aminium cation and one flurbiprofenate anion. Once again the ammonium group of the 1-phenylethan-1-aminium ion forms three charged-assisted hydrogen bonds (Table 2). The first of these bonds involves the $\mathrm{O} 2$ atom of the anion while the other two involve the $\mathrm{O} 1$ atoms of the carboxylate group of two separate symmetry-related flurbiprofenate anions. These three hydrogen bonds form a type II ladder system where each of the $\mathrm{O} 1$ atoms behaves as a bifurcated

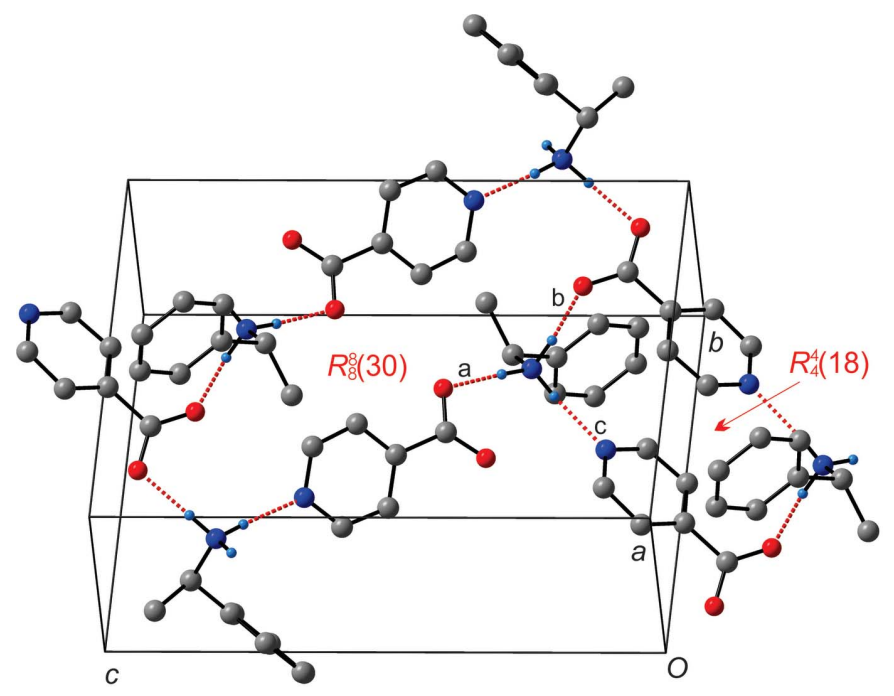

(a)

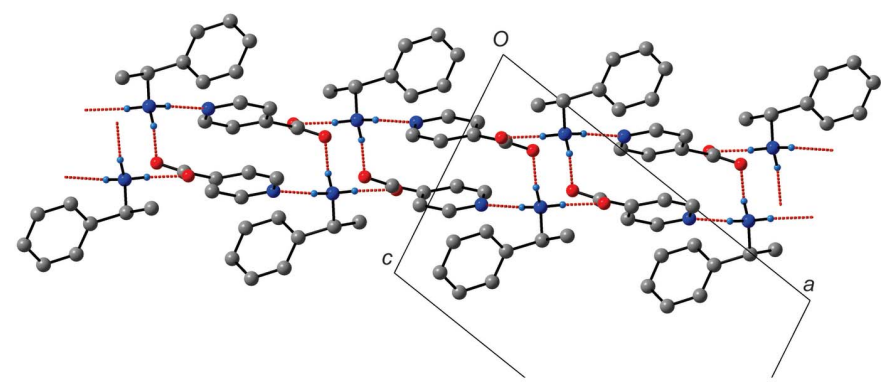

(b)

Figure 2

(a) Detailed view of the three hydrogen bonds forming two types of hydrogen-bonded rings in (I). (b) Side-on view of the two-dimensional, hydrogen-bonded layers formed.
Table 2

Hydrogen-bond geometry ( $\left.\mathrm{A},{ }^{\circ}\right)$ for (II).

\begin{tabular}{lllll}
\hline$D-\mathrm{H} \cdots A$ & $D-\mathrm{H}$ & $\mathrm{H} \cdots A$ & $D \cdots A$ & $D-\mathrm{H} \cdots A$ \\
\hline $\mathrm{N} 1-\mathrm{H} 1 A \cdots \mathrm{O} 1^{\mathrm{i}}$ & $0.91(3)$ & $1.91(3)$ & $2.809(3)$ & $170(3)$ \\
$\mathrm{N} 1-\mathrm{H} 1 C \cdots \mathrm{O} 1^{\mathrm{ii}}$ & $0.90(3)$ & $1.87(3)$ & $2.758(3)$ & $168(3)$ \\
$\mathrm{N} 1-\mathrm{H} 1 B \cdots \mathrm{O} 2$ & $0.95(3)$ & $1.75(3)$ & $2.693(3)$ & $176(3)$ \\
\hline
\end{tabular}

Symmetry codes: (i) $-x+\frac{3}{2}, y+\frac{1}{2},-z+\frac{1}{2}$; (ii) $x, y+1, z$.

Table 3

Hydrogen-bond geometry $\left(\AA,^{\circ}\right)$ for (III).

\begin{tabular}{lllll}
\hline$D-\mathrm{H} \cdots A$ & $D-\mathrm{H}$ & $\mathrm{H} \cdots A$ & $D \cdots A$ & $D-\mathrm{H} \cdots A$ \\
\hline $\mathrm{N} 1-\mathrm{H} 1 A \cdots \mathrm{O} 1$ & $0.94(2)$ & $1.91(2)$ & $2.829(1)$ & $165(1)$ \\
$\mathrm{N} 1-\mathrm{H} 1 B \cdots \mathrm{O} 2^{\mathrm{i}}$ & $0.94(2)$ & $1.85(2)$ & $2.789(1)$ & $176(1)$ \\
$\mathrm{N} 1-\mathrm{H} 1 C \cdots \mathrm{O} 1^{\mathrm{ii}}$ & $0.95(2)$ & $1.84(2)$ & $2.780(1)$ & $170(1)$ \\
\hline
\end{tabular}

Symmetry codes: (i) $x, y-1, z$; (ii) $-x+\frac{1}{2}, y-\frac{1}{2},-z+\frac{1}{2}$.

Table 4

Hydrogen-bond geometry $\left(\AA,^{\circ}\right)$ for (IV).

\begin{tabular}{lllll}
\hline$D-\mathrm{H} \cdots A$ & $D-\mathrm{H}$ & $\mathrm{H} \cdots A$ & $D \cdots A$ & $D-\mathrm{H} \cdots A$ \\
\hline $\mathrm{N} 1-\mathrm{H} 1 A \cdots \mathrm{O} 1$ & $0.88(3)$ & $1.92(3)$ & $2.796(3)$ & $175(2)$ \\
$\mathrm{N} 1-\mathrm{H} 1 B \cdots \mathrm{O}{ }^{\mathrm{i}}$ & $0.84(3)$ & $1.88(3)$ & $2.715(3)$ & $174(3)$ \\
$\mathrm{N} 1-\mathrm{H} 1 C \cdots \mathrm{O} 1^{\mathrm{ii}}$ & $0.92(3)$ & $1.83(3)$ & $2.735(2)$ & $169(3)$ \\
\hline
\end{tabular}

Symmetry codes: (i) $x, y+1, z$; (ii) $-x+\frac{3}{2}, y+\frac{1}{2},-z+\frac{1}{2}$.

hydrogen-bond acceptor, linking the rings (Fig. 3a). This pattern has translational symmetry through a twofold screw axis along the crystallographic $b$ axis which is inherent in the space group $P 2_{1} / n$. As no short contacts such as halogen bonding or $\pi$-halogen interactions are observed, the fluorine atom does not disrupt the formation of the expected hydrogen-bonding patterns. However a peculiarity exists. As the cation was present as a racemate, traditionally type III ladders are expected to dominate as reported by Lemmerer and co-workers (Lemmerer et al., 2008b).

In salt (III), the asymmetric unit consists of one 1-phenylethan-1-aminium cation and one 2-chloro-4-nitro-benzoate anion. The ammonium ion forms three charge-assisted hydrogen bonds to the carboxylate group and not to the nitro group of the 2-chloro-4-nitro-benzoate anion (Table 3). In fact, no relevant non-covalent interactions involving the nitro group are observed. As for compound (II), a type II ladder is formed by the above-mentioned hydrogen bonds, as shown in Fig. $3 b$. The anions in adjacent rings (related by translation along the $b$ axis) are connected via $\mathrm{C}-\mathrm{O} \cdots \mathrm{Cl}$ halogen bonds $\left[\mathrm{O} \cdots \mathrm{Cl}=3.225(1) \AA ; \mathrm{C}-\mathrm{O} \cdots \mathrm{Cl}=160.5(1)^{\circ}\right]$. However, this interaction does not perturb the ladder supramolecular synthons to a significant enough degree to prevent their formation. Once again, both enantiomers of the 1-phenylethan-1-aminium were present and thus type III ladders were expected to form.

In salt (IV), the asymmetric unit consists of one $\alpha$-methylbenzylammonium cation and one 4-iodobenzoate anion. A type II ladder system is observed (Table 4). An interesting feature of this structure is the $\pi \cdots$ halogen interaction between the centre of the aromatic ring of the methyl- 


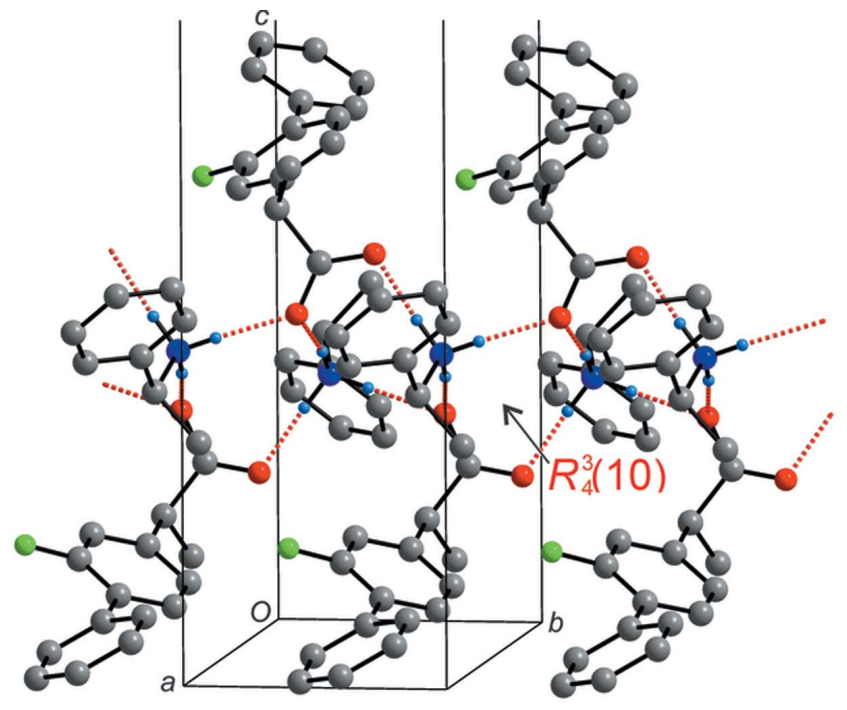

(a) (II)

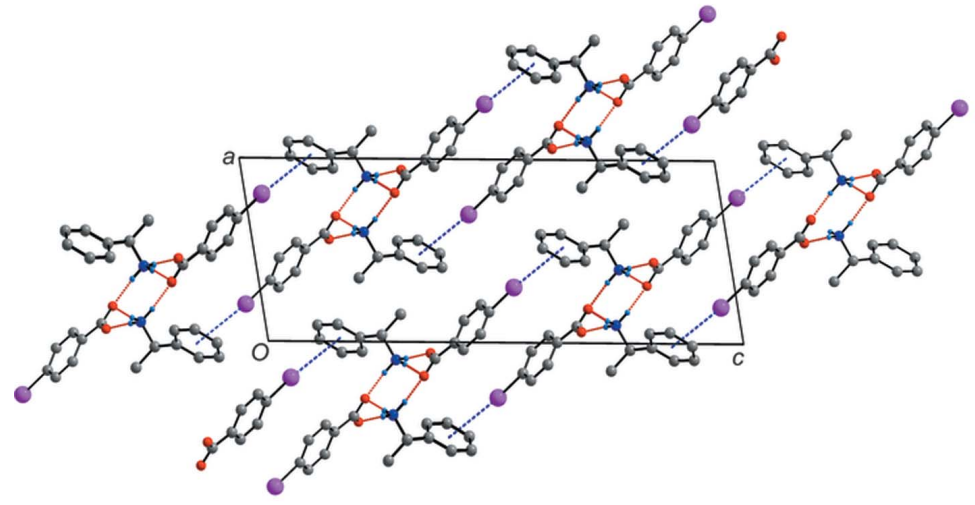

(c) (IV)

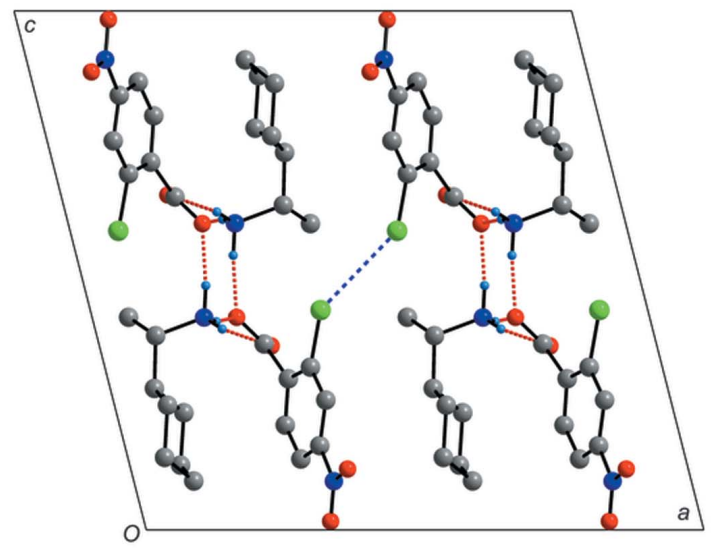

(d) $(\mathrm{V})$

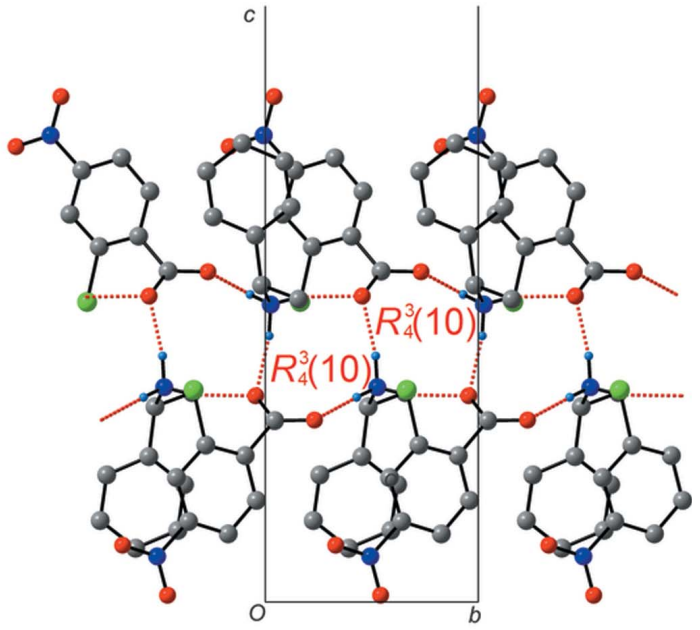

(b) (III)

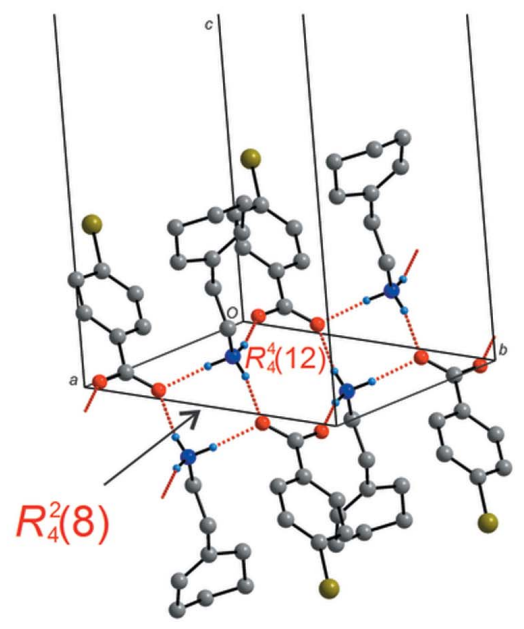

(e) (VI)

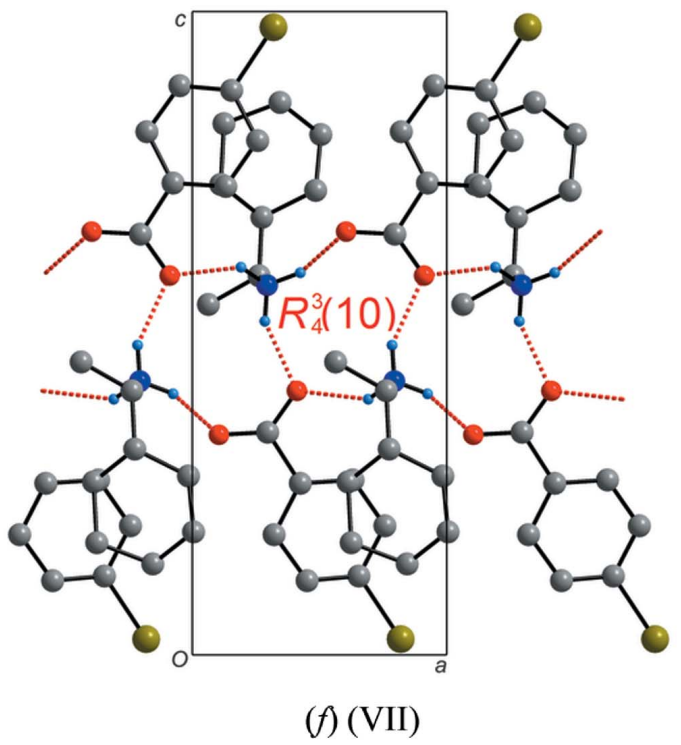

Figure 3

The hydrogen bonding (shown as dashed red lines), halogen bonding (shown as dashed blue lines) and packing diagrams for salts (II)-(VII). 
Table 5

Hydrogen-bond geometry $\left(\AA,^{\circ}\right)$ for $(\mathrm{V})$.

\begin{tabular}{lllll}
\hline$D-\mathrm{H} \cdots A$ & $D-\mathrm{H}$ & $\mathrm{H} \cdots A$ & $D \cdots A$ & $D-\mathrm{H} \cdots A$ \\
\hline $\mathrm{N} 1-\mathrm{H} 1 A \cdots \mathrm{O} 1$ & $0.93(3)$ & $1.96(3)$ & $2.869(2)$ & $167(2)$ \\
$\mathrm{N} 1-\mathrm{H} 1 B \cdots \mathrm{O} 1^{\mathrm{i}}$ & $0.93(3)$ & $1.86(3)$ & $2.785(2)$ & $173(2)$ \\
$\mathrm{N} 1-\mathrm{H} 1 C \cdots \mathrm{O} 2^{\mathrm{ii}}$ & $0.88(3)$ & $1.99(3)$ & $2.858(2)$ & $170(2)$ \\
\hline
\end{tabular}

Symmetry codes: (i) $-x+\frac{3}{2}, y-\frac{1}{2},-z+1$; (ii) $x, y-1, z$.

Table 6

Hydrogen-bond geometry $\left(\AA,^{\circ}\right)$ for (VI).

\begin{tabular}{lllll}
\hline$D-\mathrm{H} \cdots A$ & $D-\mathrm{H}$ & $\mathrm{H} \cdots A$ & $D \cdots A$ & $D-\mathrm{H} \cdots A$ \\
\hline $\mathrm{N} 1-\mathrm{H} 1 A \cdots \mathrm{O} 1$ & $0.86(3)$ & $1.89(3)$ & $2.737(2)$ & $167(3)$ \\
$\mathrm{N} 1-\mathrm{H} 1 B \cdots \mathrm{O} 2^{\mathrm{i}}$ & $0.94(3)$ & $1.85(3)$ & $2.763(3)$ & $164(3)$ \\
$\mathrm{N} 1-\mathrm{H} 1 C \cdots \mathrm{O} 2^{\mathrm{ii}}$ & $0.86(3)$ & $1.89(3)$ & $2.727(2)$ & $167(3)$ \\
\hline
\end{tabular}

Symmetry codes: (i) $x+1, y, z$; (ii) $-x+1,-y+1,-z$.

Table 7

Hydrogen-bond geometry $\left(\AA{ }^{\circ}\right)$ for (VII).

\begin{tabular}{lllll}
\hline$D-\mathrm{H} \cdots A$ & $D-\mathrm{H}$ & $\mathrm{H} \cdots A$ & $D \cdots A$ & $D-\mathrm{H} \cdots A$ \\
\hline $\mathrm{N} 1-\mathrm{H} 1 A \cdots \mathrm{O} 1$ & 0.91 & 1.99 & $2.781(12)$ & 144 \\
$\mathrm{~N} 1-\mathrm{H} 1 B \cdots \mathrm{O} 2^{\mathrm{i}}$ & 0.91 & 2.06 & $2.870(12)$ & 148 \\
$\mathrm{~N} 1-\mathrm{H} 1 C \cdots \mathrm{O} 1^{\mathrm{ii}}$ & 0.91 & 1.89 & $2.718(10)$ & 150 \\
\hline
\end{tabular}

Symmetry codes: (i) $x+1, y, z$; (ii) $x+\frac{1}{2},-y+\frac{1}{2},-z+1$.

benzylammonium cation and the iodine atom (Fig. 3c). This is possible as, due to its size, iodine is very polarizable and thus the delocalized electrons in the aromatic system can create a permanent dipole in the iodine atom in the solid state. The distance of 3.740 (3) $\AA$ is similar to other molecules containing iodine interacting non-covalently with aromatic systems reported in the literature (Nagels et al., 2013). Again, as in salt (III), the halogen bonding does not disrupt the formation of the ladder motif.

In salt (V), the asymmetric unit consists of one $(S)$-1cyclohexylethylammonium cation and one 2-chloro-4-nitrobenzoate anion, both on general positions. A type II ladder is formed as shown in Fig. $3 d$. No hydrogen bonding to the nitro group takes place (Table 5), which is consistent with the results for salt (III). However, a type $\mathrm{I} \mathrm{Cl} \cdots \mathrm{Cl}$ halogen bond is observed with a distance of 3.207 (3) $⿱$ A that connects adjacent ladders along the $a$ axis. As the cation is present as a single enantiomer, the type II ladder formation is in line with the previous studies.

In salt (VI), the asymmetric unit consists of one 2-(1cyclohexenyl)ethylammonium cation and one 4-bromobenzoate anion, both on general positions. A type III ladder is observed (Table 6), unique among the salts here reported (Fig. 3e). As in salt (V), the crystal structure is stabilized by halogen bonding, in this case between bromine and oxygen $\mathrm{O} 1$ with a distance of 3.253 (3) $\AA$. The halogen bond connects adjacent ladders related by the two-fold screw axis.

In salt (VII), the asymmetric unit consists of one $(S)-1$ cyclohexylethylammonium cation and one 4-bromobenzoate anion, both on general positions. A type II ladder is formed (Table 7, Fig. 3f). This is expected as the cation is enanti- omerically pure (Lemmerer et al., 2008b). In contrast to the previous salts that have a halogen atom on the anion, no halogen bonding is observed.

In summary, introducing a pyridine functional group instead of a plain benzene in (I) has altered the hydrogen-bonding pattern usually observed in ammonium carboxylate salts, which generally show the typical type II and III patterns as seen in (II)-(VII)

\section{Synthesis and crystallization}

All chemicals were purchased from commercial sources (Sigma Aldrich) and used as received without further purification. Crystals were grown via the slow evaporation of methanol or ethanol solutions under ambient conditions. All solutions contained a 1:1 ratio of amine and acid. Detailed masses and volumes are as follows. For (I): $(R S)$ - $\alpha$-methylbenzylamine $(0.100 \mathrm{~g}, 0.825 \mathrm{mmol})$ and isonicotinic acid (0.102 g, $0.825 \mathrm{mmol})$ in methanol $(5 \mathrm{~mL})$; for (II): $(R S)-\alpha-$ methylbenzylamine $(0.100 \mathrm{~g}, 0.825 \mathrm{mmol})$ and flurbiprofen $(0.202 \mathrm{~g}, 0.825 \mathrm{mmol})$ in ethanol $(8 \mathrm{~mL})$; for (III): $(R S)-\alpha-$ methylbenzylamine $(0.100 \mathrm{~g}, 0.825 \mathrm{mmol})$ and 2-chloro-4nitro-benzoic acid $(0.166 \mathrm{~g}, 0.825 \mathrm{mmol})$ in ethanol $(5 \mathrm{~mL})$; for (IV): $(R S)-\alpha$-methylbenzylamine $(0.492 \mathrm{~g}, 0.406 \mathrm{mmol})$ and 4-iodobenzoic acid $(0.101 \mathrm{~g}, 0.406 \mathrm{mmol})$ in ethanol $(5 \mathrm{~mL})$; for (V): (S)-1-cyclohexylethylamine $(0.0254 \mathrm{~g}, 0.200 \mathrm{mmol})$ and 2-chloro-4-nitro-benzoic acid $(0.0403 \mathrm{~g}, 0.200 \mathrm{mmol})$; for (VI): 2-(1-cyclohexenyl) ethylamine $(0.0250 \mathrm{~g}, 0.200 \mathrm{mmol})$ and 4-bromobenzoic acid $(0.0410 \mathrm{~g}, 0.200 \mathrm{mmol})$; and for (VII): (S)-1-cyclohexylethylamine $(0.0254 \mathrm{~g}, 0.200 \mathrm{mmol})$ and 4-bromobenzoic acid $(0.0410 \mathrm{~g}, 0.200 \mathrm{mmol})$.

\section{Refinement details}

Crystal data, data collection and structure refinement details are summarized in Table 8 . For all compounds, the C-bound $\mathrm{H}$ atoms were placed geometrically $[\mathrm{C}-\mathrm{H}$ bond lengths of $1.00 \AA$ (methine $\mathrm{CH}$ ), $0.99 \AA$ (ethylene $\mathrm{CH}_{2}$ ), $0.98 \AA$ (methylene $\mathrm{CH}_{3}$ ) and $\left.0.95 \AA(\mathrm{Ar}-\mathrm{H})\right]$ and refined as riding with $U_{\text {iso }}(\mathrm{H})=1.2 U_{\text {eq }}(\mathrm{C})$ or $U_{\text {iso }}(\mathrm{H})=1.5 U_{\text {eq }}(\mathrm{C})$. The N-bound $\mathrm{H}$ atoms were located in difference-Fourier maps and their coordinates and isotropic displacement parameters allowed to refine freely for (I)-(VI). For (VII), the N-bound $\mathrm{H}$ atoms were geometrically placed $(\mathrm{C}-\mathrm{H}$ bond lengths of $0.91 \AA)$ and refined as riding with $U_{\text {iso }}(\mathrm{H})=1.5 U_{\text {eq }}(\mathrm{C})$.

For the disorder of the atom $\mathrm{C} 4$ in the cyclohexene ring of (VI), two alternate positions were found in a differenceFourier map, and their occupancies refined to final values of 0.77 (2) and $0.23(2)$.

\section{Related literature}

The following references, not cited in the main body of the paper, have been cited in the supporting information: Bouchard et al. (2002); Isoda et al. (1997); Perrin (1982); Portnov et al. (1971); van Sorge et al. (1999); Tuckerman et al. (1959). 
Table 8

Experimental details.

(I)

(II)

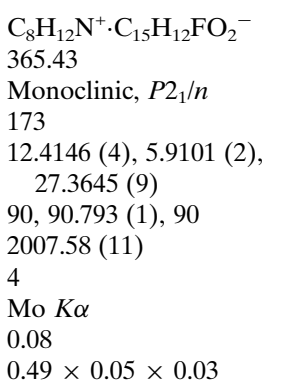

(III)

(IV)

Crystal data

Chemical formula

$M_{\mathrm{r}}$

Crystal system, space group

Temperature (K)

$a, b, c(\AA)$

$\alpha, \beta, \gamma\left(^{\circ}\right)$

$V\left(\AA^{3}\right)$

$Z$

Radiation type

$\mu\left(\mathrm{mm}^{-1}\right)$

Crystal size (mm)

$\mathrm{C}_{8} \mathrm{H}_{12} \mathrm{~N}^{+} \cdot \mathrm{C}_{6} \mathrm{H}_{4} \mathrm{NO}_{2}{ }^{-}$ 244.29

Monoclinic, $P 2_{1} / n$

173

9.4094 (5), 9.4697 (5),

$15.1613(9)$

90, 102.247 (3), 90

1320.19 (13)

4

Mo $K \alpha$

0.08

$0.76 \times 0.33 \times 0.07$

$0.49 \times 0.05 \times 0.03$

Bruker D8 Venture Photon CCD area detector

Diffractometer

Absorption correction

$T_{\min }, T_{\max }$ 2016)

$0.95,0.96$

No. of measured, independent 38822, 3190, 2359 and observed $[I>2 \sigma(I)]$ reflections

0.101

Refinement

$R\left[F^{2}>2 \sigma\left(F^{2}\right)\right], w R\left(F^{2}\right), S$

No. of reflections

No. of parameters

No. of restraints

$\mathrm{H}$-atom treatment

$\Delta \rho_{\max }, \Delta \rho_{\min }\left(\mathrm{e} \AA^{-3}\right)$

$0.056,0.171,1.05$
3190
176
0
H atoms treated by a mixture
$\quad$ of independent and
$\quad$ constrained refinement
$0.38,-0.40$

Bruker D8 Venture Photon $\mathrm{CCD}$ area detector Integration (XPREP; Bruker, 2016) $0.984,0.998$

$21119,3729,3014$

0.036

$0.061,0.174,1.07$

3729

256

0

$\mathrm{H}$ atoms treated by a mixture of independent and constrained refinement $1.00,-0.30$

$\mathrm{C}_{8} \mathrm{H}_{12} \mathrm{~N}^{+} \cdot \mathrm{C}_{7} \mathrm{H}_{3} \mathrm{ClNO}_{4}{ }^{-}$
322.74
Monoclinic, $C 2 / c$
173
$15.5817(7), 6.3914(3)$,
$\quad 31.3238(14)$
$90,100.998(2), 90$
$3062.2(2)$
8
Mo $K \alpha$
0.27
$0.47 \times 0.35 \times 0.08$

$\mathrm{C}_{8} \mathrm{H}_{12} \mathrm{~N}^{+} \cdot \mathrm{C}_{7} \mathrm{H}_{4} \mathrm{IO}_{2}{ }^{-}$

369.19

Monoclinic, $P 2_{1} / n$

173

9.7224 (5), $6.0571(3)$

24.8767 (12)

90, $99.527(2), 90$

1444.77 (12)

4

Mo $K \alpha$

2.21

$0.68 \times 0.16 \times 0.04$

\section{(V)}

Crystal data

Chemical formula

$M_{\mathrm{r}}$

Crystal system, space group

Temperature (K)

$a, b, c(\AA)$

\section{$\alpha, \beta, \gamma\left({ }^{\circ}\right)$ \\ $V\left(\AA^{3}\right)$}

Z

Radiation type

$\mu\left(\mathrm{mm}^{-1}\right)$

Crystal size (mm)

Data collection

Diffractometer

Absorption correction

$T_{\min }, T_{\max }$

No. of measured, independent and observed $[I>2 \sigma(I)]$ reflections

$R_{\text {int }}$

\begin{tabular}{|c|}
\hline $\begin{array}{l}\mathrm{C}_{8} \mathrm{H}_{18} \mathrm{~N}^{+} \cdot \mathrm{C}_{7} \mathrm{H}_{3} \mathrm{ClNO}_{4}{ }^{-} \\
328.79\end{array}$ \\
\hline Monoclinic, $C 2$ \\
\hline 173 \\
\hline $\begin{array}{l}16.2280(15), 6.4392(5), \\
\quad 15.5937(15)\end{array}$ \\
\hline $90,104.289(4), 90$ \\
\hline $1579.1(2)$ \\
\hline \\
\hline Mo $K \alpha$ \\
\hline \\
\hline
\end{tabular}

Bruker D8 Venture Photon CCD area detector

Integration (XPREP; Bruker, 2016)

$0.910,0.988$

$15055,3837,3587$

0.045

$0.032,0.071,1.04$

3837

211

1

$\mathrm{H}$ atoms treated by a mixture of independent and constrained refinement

$0.20,-0.18$

(VI)

\section{$\mathrm{C}_{8} \mathrm{H}_{15} \mathrm{~N}^{+} \cdot \mathrm{C}_{7} \mathrm{H}_{4} \mathrm{BrO}_{2}^{-}$ \\ 325.22 \\ Monoclinic, $P 2_{1} / n$ \\ 173 \\ 90, $102.241(2), 90$ \\ $1514.86(12)$ \\ 4 \\ Mo $K \alpha$ \\ 2.71 \\ $0.68 \times 0.18 \times 0.1$}

6.4391 (3), 17.0023 (8), 14.1588 (6)

Bruker D8 Venture Photon CCD area detector

Integration (XPREP; Bruker, 2016)

$0.275,0.776$

$30862,3658,3302$

0.071

0.037, 0.098, 1.05

3658

194

0

$\mathrm{H}$ atoms treated by a mixture of independent and constrained refinement

$1.01,-1.02$

\section{(VII)}

Bruker D8 Venture Photon $\mathrm{CCD}$ area detector Integration (XPREP; Bruker, 2016) $0.508,0.928$

30978, 3463, 3109

0.051

$0.026,0.057,1.13$

3463

185

0

$\mathrm{H}$ atoms treated by a mixture of independent and constrained refinement $0.74,-0.38$

No. of restraints

$\Delta \rho_{\max }, \Delta \rho_{\min }\left(\mathrm{e} \AA^{-3}\right)$

$\mathrm{C}_{8} \mathrm{H}_{18} \mathrm{~N}^{+} \cdot \mathrm{C}_{7} \mathrm{H}_{4} \mathrm{BrO}_{2}^{-}$

Orthorhombic, $P 2{ }_{1} 22_{1}$

173

6.2790 (3), $15.6610(9), 15.8800$ (8)

90, 90, 90

1561.57 (14)

4

Mo $K \alpha$

2.63

$0.69 \times 0.13 \times 0.10$

Bruker D8 Venture Photon CCD area detector

Integration (XPREP; Bruker, 2016)

$0.452,0.846$

21006, 2913, 2687

0.068

$0.078,0.211,1.08$

2913

174

$\mathrm{H}$-atom parameters constrained

$1.46,-0.48$ 
Table 8 (continued)

\begin{tabular}{llcc}
\hline & $(\mathrm{V})$ & $(\mathrm{VI})$ & $(\mathrm{VII})$ \\
\hline Absolute structure & Flack $x$ determined using 1512 & - & Flack $x$ determined using 1026 \\
& quotients $\left[\left(I^{+}\right)-\left(I^{-}\right)\right] /\left[\left(I^{+}\right)+\left(I^{-}\right)\right]$ & & quotients $\left[\left(I^{+}\right)-\left(I^{-}\right)\right] /\left[\left(I^{+}\right)+\left(I^{-}\right)\right]$ \\
& $\quad$ (Parsons et al., 2013). & - & $($ Parsons et al., 2013) \\
Absolute structure parameter & $-0.031(19)$ & $0.068(9)$ & \\
\hline
\end{tabular}

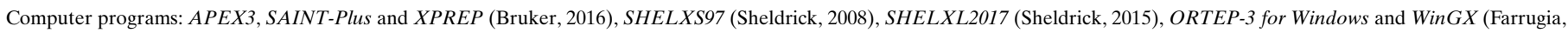
2012) and DIAMOND (Brandenburg \& Berndt, 1999).

\section{Funding information}

This material is based upon work supported financially by the University of the Witwatersrand Friedel Sellschop Grant and the Molecular Sciences Institute. The National Research Foundation National Equipment Programme (UID: 78572) is thanked for financing the purchase of the single-crystal diffractometer. Any opinions, findings and conclusions or recommendations expressed in this material are those of the authors and therefore the NRF does not accept any liability in regard thereto.

\section{References}

Bernstein, J., Davis, R. E., Shimoni, L. \& Chang, N. L. (1995). Angew. Chem. Int. Ed. Engl. 34, 1555-1573.

Bhogala, B. R., Basavoju, S. \& Nangia, A. (2005). CrystEngComm, 7, 551-562.

Bouchard, G., Carrupt, P.-A., Testa, B., Gobry, V. \& Girault, H. H. (2002). Chem. Eur. J. 8, 3478-3484.

Brandenburg, K. \& Berndt, M. (1999). DIAMOND. Crystal Impact GbR, Bonn, Germany.

Bruker (2016). APEX3, SAINT-Plus and XPREP. Bruker AXS Inc., Madison, Wisconsin, USA.

Desiraju, G. R. (1989). Crystal Engineering: The Design of Organic Solids. Amsterdam: Elsevier.

Farrugia, L. J. (2012). J. Appl. Cryst. 45, 849-854.
Isoda, T., Yamasaki, M., Yano, H. \& Harada, S. (1997). Faraday Trans. 93, 449-452.

Kinbara, K., Hashimoto, Y., Sukegawa, M., Nohira, H. \& Saigo, K. (1996). J. Am. Chem. Soc. 118, 3441-3449.

Lemmerer, A., Bourne, S. A. \& Fernandes, M. A. (2008a). Cryst. Growth Des. 8, 1106-1109.

Lemmerer, A., Bourne, S. A. \& Fernandes, M. A. (2008b). CrystEngComm, 10, 1605-1612.

Lemmerer, A., Govindraju, S., Johnston, M., Motloung, X. \& Savig, K. L. (2015). CrystEngComm, 17, 3591-3595.

Nagels, N., Hauchecorne, D. \& Herrebout, W. (2013). Molecules, 18, 6829-6851.

Odendal, J. A., Bruce, J. C., Koch, K. R. \& Haynes, D. A. (2010). CrystEngComm, 12, 2398-2408.

Odom, S. A., Parkin, S. R. \& Anthony, J. E. (2003). Org. Lett. 5, 42454248.

Parsons, S., Flack, H. D. \& Wagner, T. (2013). Acta Cryst. B69, 249259.

Perrin, D. D. (1982). Ionization Constants of Organic Acids and Bases in Aqueous Solution, 2nd edition. Oxford: Pergamon.

Portnov, M. A., Al'tshuler, G. N., Vaisman, M. N., Dubinina, T. A. \& Yakhontov, L. N. (1971). Pharm. Chem. J. 5, 426-428.

Sheldrick, G. M. (2008). Acta Cryst. A64, 112-122.

Sheldrick, G. M. (2015). Acta Cryst. C71, 3-8.

Snaith, H. J. (2013). J. Phys. Chem. Lett. 4, 3623-3630.

Sorge, A. A. van, Wijnen, P., van Delft, J., Carballosa Coré-Bodelier, V. M. W. \& van Haeringen, N. (1999). Pharm. World Sci. 21, 91-95. Tessler, N. (1999). Adv. Mater. 11, 363-370.

Tuckerman, M. M., Mayer, J. R. \& Nachod, F. C. (1959). J. Am. Chem. Soc. 81, 92-94. 


\section{supporting information}

Acta Cryst. (2018). E74, 580-586 [https://doi.org/10.1107/S2056989017017856]

\section{An investigation to elucidate the factors dictating the crystal structure of seven ammonium carboxylate molecular salts}

\section{Jacques Blignaut and Andreas Lemmerer}

\section{Computing details}

For all structures, data collection: APEX3 (Bruker, 2016); cell refinement: SAINT-Plus (Bruker, 2016); data reduction: SAINT-Plus (Bruker, 2016) and XPREP (Bruker, 2016); program(s) used to solve structure: SHELXS97 (Sheldrick, 2008); program(s) used to refine structure: SHELXL2017 (Sheldrick, 2015); molecular graphics: ORTEP-3 for Windows (Farrugia, 2012) and DIAMOND (Brandenburg \& Berndt, 1999); software used to prepare material for publication:

WinGX (Farrugia, 2012).

(RS)-1-Phenylethan-1-aminium pyridine-4-carboxylate (I)

Crystal data

$$
\begin{aligned}
& \mathrm{C}_{8} \mathrm{H}_{12} \mathrm{~N}^{+} \cdot \mathrm{C}_{6} \mathrm{H}_{4} \mathrm{NO}_{2}{ }^{-} \\
& M_{r}=244.29 \\
& \text { Monoclinic, } P 2_{1} / n \\
& \text { Hall symbol: }-\mathrm{P} 2 \mathrm{yn} \\
& a=9.4094(5) \AA \\
& b=9.4697(5) \AA \\
& c=15.1613(9) \AA \\
& \beta=102.247(3)^{\circ} \\
& V=1320.19(13) \AA^{3} \\
& Z=4
\end{aligned}
$$

\section{Data collection}

Bruker D8 Venture Photon CCD area detector diffractometer

Graphite monochromator $\omega$ scans

Absorption correction: integration (XPREP; Bruker, 2016)

$T_{\min }=0.95, T_{\max }=0.96$

38822 measured reflections

\section{Refinement}

Refinement on $F^{2}$ Least-squares matrix: full $R\left[F^{2}>2 \sigma\left(F^{2}\right)\right]=0.056$ $w R\left(F^{2}\right)=0.171$ $S=1.05$ 3190 reflections 176 parameters
$F(000)=520$

$D_{\mathrm{x}}=1.229 \mathrm{Mg} \mathrm{m}^{-3}$

Mo $K \alpha$ radiation, $\lambda=0.71073 \AA$

Cell parameters from 8190 reflections

$\theta=2.4-24.1^{\circ}$

$\mu=0.08 \mathrm{~mm}^{-1}$

$T=173 \mathrm{~K}$

Plate, colourless

$0.76 \times 0.33 \times 0.07 \mathrm{~mm}$

3190 independent reflections 2359 reflections with $I>2 \sigma(I)$

$R_{\text {int }}=0.101$

$\theta_{\text {max }}=28.0^{\circ}, \theta_{\text {min }}=2.4^{\circ}$

$h=-12 \rightarrow 12$

$k=-12 \rightarrow 12$

$l=-20 \rightarrow 20$

0 restraints

0 constraints

Hydrogen site location: mixed

$\mathrm{H}$ atoms treated by a mixture of independent and constrained refinement

$w=1 /\left[\sigma^{2}\left(F_{\mathrm{o}}^{2}\right)+(0.0941 P)^{2}+0.2604 P\right]$ where $P=\left(F_{\mathrm{o}}^{2}+2 F_{\mathrm{c}}{ }^{2}\right) / 3$ 
$(\Delta / \sigma)_{\max }<0.001$

$\Delta \rho_{\max }=0.38$ e $\AA^{-3}$

$$
\Delta \rho_{\min }=-0.40 \text { e } \AA^{-3}
$$

Special details

Experimental. Numerical integration absorption corrections based on indexed crystal faces were applied using the XPREP routine (Bruker, 2016)

Geometry. All esds (except the esd in the dihedral angle between two 1.s. planes) are estimated using the full covariance matrix. The cell esds are taken into account individually in the estimation of esds in distances, angles and torsion angles; correlations between esds in cell parameters are only used when they are defined by crystal symmetry. An approximate (isotropic) treatment of cell esds is used for estimating esds involving l.s. planes.

Fractional atomic coordinates and isotropic or equivalent isotropic displacement parameters $\left(\AA^{2}\right)$

\begin{tabular}{|c|c|c|c|c|}
\hline & $x$ & $y$ & $z$ & $U_{\text {iso }} * / U_{\text {eq }}$ \\
\hline $\mathrm{C} 1$ & $0.36397(17)$ & $0.73101(15)$ & $0.23522(11)$ & $0.0356(4)$ \\
\hline $\mathrm{C} 2$ & $0.37700(18)$ & $0.82392(16)$ & $0.16665(12)$ & 0.0379 \\
\hline $\mathrm{H} 2$ & 0.469713 & 0.861634 & 0.164378 & $0.045^{*}$ \\
\hline $\mathrm{C} 3$ & 0.25688 & $0.86235(18)$ & $0.10161(13)$ & $0.0427(4)$ \\
\hline $\mathrm{H} 3$ & 0.267388 & 0.924437 & 0.054193 & $0.051^{*}$ \\
\hline $\mathrm{C} 4$ & $0.1213(2)$ & $0.8100(2)$ & $0.10587(13)$ & 0.0475 \\
\hline $\mathrm{H} 4$ & 0.038141 & 0.837452 & 0.06192 & $0.057^{*}$ \\
\hline $\mathrm{C} 5$ & $0.1068(2)$ & $0.7180(2)$ & $0.17387(15)$ & $0.0536(5)$ \\
\hline H5 & 0.013626 & 0.68193 & 0.176604 & $0.064^{*}$ \\
\hline C6 & $0.22735(19)$ & $0.6783(2)$ & $0.23802(14)$ & $0.0471(4)$ \\
\hline H6 & 0.216714 & 0.614276 & 0.284453 & $0.057^{*}$ \\
\hline $\mathrm{C} 7$ & $0.49410(18)$ & $0.68555(16)$ & $0.30626(11)$ & $0.0368(4)$ \\
\hline $\mathrm{H} 7$ & 0.45852 & 0.621973 & 0.349619 & $0.044^{*}$ \\
\hline $\mathrm{C} 8$ & $0.5745(2)$ & $0.80785(18)$ & $0.35973(13)$ & $0.0482(5)$ \\
\hline H8A & 0.657613 & 0.771497 & 0.404167 & $0.072 *$ \\
\hline H8B & 0.508569 & 0.858155 & 0.390937 & $0.072 *$ \\
\hline $\mathrm{H} 8 \mathrm{C}$ & 0.609192 & 0.872738 & 0.318543 & $0.072 *$ \\
\hline $\mathrm{C} 10$ & 0.71054 (19) & $0.61304(16)$ & $0.01922(13)$ & $0.0421(4)$ \\
\hline H10 & 0.783407 & 0.575032 & 0.066354 & $0.051 *$ \\
\hline C11 & $0.6532(2)$ & $0.53314(17)$ & $-0.05587(13)$ & 0.0475 \\
\hline H11 & 0.690245 & 0.440511 & -0.059585 & $0.057^{*}$ \\
\hline $\mathrm{C} 12$ & $0.49964(18)$ & $0.71011(17)$ & $-0.11664(12)$ & 0.0389 \\
\hline H12 & 0.424163 & 0.744187 & -0.163695 & $0.047^{*}$ \\
\hline $\mathrm{C} 13$ & $0.55272(17)$ & $0.79860(15)$ & $-0.04510(11)$ & $0.0363(4)$ \\
\hline $\mathrm{H} 13$ & 0.515779 & 0.891799 & -0.044027 & $0.044^{*}$ \\
\hline $\mathrm{C} 14$ & $0.66020(16)$ & $0.75029(15)$ & $0.02501(11)$ & 0.0320 \\
\hline $\mathrm{C} 15$ & $0.72087(15)$ & $0.84180(15)$ & $0.10609(11)$ & $0.0312(3)$ \\
\hline N1 & $0.59829(15)$ & $0.60289(13)$ & $0.26409(10)$ & $0.0340(3)$ \\
\hline $\mathrm{N} 2$ & $0.54883(16)$ & $0.57833(14)$ & $-0.12346(10)$ & $0.0420(4)$ \\
\hline $\mathrm{O} 1$ & $0.76355(12)$ & $0.78218(11)$ & $0.18042(8)$ & 0.0382 \\
\hline $\mathrm{O} 2$ & $0.72283(14)$ & $0.97236(11)$ & $0.09234(8)$ & 0.0446 \\
\hline H1A & $0.549(2)$ & $0.541(2)$ & $0.2211(14)$ & $0.047(5)^{*}$ \\
\hline H1B & $0.664(2)$ & $0.560(2)$ & $0.3127(15)$ & $0.052(6) *$ \\
\hline $\mathrm{H} 1 \mathrm{C}$ & $0.651(2)$ & $0.666(2)$ & $0.2308(13)$ & $0.048(5)^{*}$ \\
\hline
\end{tabular}


Atomic displacement parameters $\left(\AA^{2}\right)$

\begin{tabular}{lllllll}
\hline & $U^{11}$ & $U^{22}$ & $U^{33}$ & $U^{12}$ & $U^{13}$ & $U^{23}$ \\
\hline C1 & $0.0372(8)$ & $0.0231(7)$ & $0.0463(9)$ & $0.0016(6)$ & $0.0086(7)$ & $-0.0025(6)$ \\
C2 & $0.0364(8)$ & $0.0255(7)$ & $0.0519(10)$ & $-0.0001(6)$ & $0.0096(7)$ & $0.0012(6)$ \\
C3 & $0.0484(10)$ & $0.0293(8)$ & $0.0496(10)$ & $0.0068(7)$ & $0.0083(8)$ & $0.0036(7)$ \\
C4 & $0.0390(9)$ & $0.0436(10)$ & $0.0559(11)$ & $0.0110(7)$ & $0.0014(8)$ & $-0.0045(8)$ \\
C5 & $0.0341(9)$ & $0.0560(12)$ & $0.0707(13)$ & $-0.0025(8)$ & $0.0108(9)$ & $0.0024(10)$ \\
C6 & $0.0417(10)$ & $0.0423(9)$ & $0.0589(11)$ & $-0.0042(7)$ & $0.0140(8)$ & $0.0066(8)$ \\
C7 & $0.0404(9)$ & $0.0261(7)$ & $0.0431(9)$ & $0.0004(6)$ & $0.0069(7)$ & $0.0009(6)$ \\
C8 & $0.0546(11)$ & $0.0343(9)$ & $0.0517(11)$ & $0.0016(7)$ & $0.0022(8)$ & $-0.0112(7)$ \\
C10 & $0.0445(9)$ & $0.0232(7)$ & $0.0519(10)$ & $0.0068(6)$ & $-0.0048(7)$ & $-0.0022(7)$ \\
C11 & $0.0547(10)$ & $0.0228(8)$ & $0.0590(11)$ & $0.0073(7)$ & $-0.0015(9)$ & $-0.0078(7)$ \\
C12 & $0.0375(8)$ & $0.0281(8)$ & $0.0467(9)$ & $0.0012(6)$ & $-0.0008(7)$ & $-0.0020(6)$ \\
C13 & $0.0375(8)$ & $0.0208(7)$ & $0.0477(9)$ & $0.0031(6)$ & $0.0024(7)$ & $-0.0002(6)$ \\
C14 & $0.0313(7)$ & $0.0194(6)$ & $0.0442(8)$ & $-0.0012(5)$ & $0.0055(6)$ & $0.0002(6)$ \\
C15 & $0.0279(7)$ & $0.0211(7)$ & $0.0429(8)$ & $0.0007(5)$ & $0.0033(6)$ & $0.0001(6)$ \\
N1 & $0.0360(7)$ & $0.0182(6)$ & $0.0443(8)$ & $0.0009(5)$ & $0.0006(6)$ & $0.0011(5)$ \\
N2 & $0.0465(8)$ & $0.0250(7)$ & $0.0510(8)$ & $-0.0025(6)$ & $0.0026(6)$ & $-0.0068(6)$ \\
O1 & $0.0390(6)$ & $0.0294(6)$ & $0.0423(7)$ & $-0.0036(4)$ & $0.0000(5)$ & $0.0034(5)$ \\
O2 & $0.0582(8)$ & $0.0181(5)$ & $0.0509(7)$ & $-0.0017(5)$ & $-0.0036(6)$ & $-0.0010(5)$ \\
& & & & & &
\end{tabular}

Geometric parameters $\left(\AA,{ }^{\circ}\right)$

\begin{tabular}{llll}
\hline $\mathrm{C} 1-\mathrm{C} 2$ & $1.387(2)$ & $\mathrm{C} 8-\mathrm{H} 8 \mathrm{C}$ & 0.98 \\
$\mathrm{C} 1-\mathrm{C} 6$ & $1.388(2)$ & $\mathrm{C} 10-\mathrm{C} 11$ & $1.378(2)$ \\
$\mathrm{C} 1-\mathrm{C} 7$ & $1.512(2)$ & $\mathrm{C} 10-\mathrm{C} 14$ & $1.392(2)$ \\
$\mathrm{C} 2-\mathrm{C} 3$ & $1.382(2)$ & $\mathrm{C} 10-\mathrm{H} 10$ & 0.95 \\
$\mathrm{C} 2-\mathrm{H} 2$ & 0.95 & $\mathrm{C} 11-\mathrm{N} 2$ & $1.331(2)$ \\
$\mathrm{C} 3-\mathrm{C} 4$ & $1.383(3)$ & $\mathrm{C} 11-\mathrm{H} 11$ & 0.95 \\
$\mathrm{C} 3-\mathrm{H} 3$ & 0.95 & $\mathrm{C} 12-\mathrm{N} 2$ & $1.343(2)$ \\
$\mathrm{C} 4-\mathrm{C} 5$ & $1.378(3)$ & $\mathrm{C} 12-\mathrm{C} 13$ & $1.378(2)$ \\
$\mathrm{C} 4-\mathrm{H} 4$ & 0.95 & $\mathrm{C} 12-\mathrm{H} 12$ & 0.95 \\
$\mathrm{C} 5-\mathrm{C} 6$ & $1.381(3)$ & $\mathrm{C} 13-\mathrm{C} 14$ & $1.381(2)$ \\
$\mathrm{C} 5-\mathrm{H} 5$ & 0.95 & $\mathrm{C} 13-\mathrm{H} 13$ & 0.95 \\
$\mathrm{C} 6-\mathrm{H} 6$ & 0.95 & $\mathrm{C} 14-\mathrm{C} 15$ & $1.513(2)$ \\
$\mathrm{C} 7-\mathrm{N} 1$ & $1.500(2)$ & $\mathrm{C} 15-\mathrm{O} 1$ & $1.2482(18)$ \\
$\mathrm{C} 7-\mathrm{C} 8$ & $1.520(2)$ & $\mathrm{C} 15-\mathrm{O} 2$ & $0.93(2)$ \\
$\mathrm{C} 7-\mathrm{H} 7$ & 1 & $\mathrm{~N} 1-\mathrm{H} 1 \mathrm{~A}$ & $0.95(2)$ \\
$\mathrm{C} 8-\mathrm{H} 8 \mathrm{~A}$ & 0.98 & $\mathrm{~N} 1-\mathrm{H} 1 \mathrm{~B}$ & $0.98(2)$ \\
$\mathrm{C} 8-\mathrm{H} 8 \mathrm{~B}$ & 0.98 & $\mathrm{~N} 1-\mathrm{H} 1 \mathrm{C}$ & 109.5 \\
& & & 109.5 \\
$\mathrm{C} 2-\mathrm{C} 1-\mathrm{C} 6$ & $118.74(16)$ & $\mathrm{H} 8 \mathrm{~A}-\mathrm{C} 8-\mathrm{H} 8 \mathrm{C}$ & $119.06(16)$ \\
$\mathrm{C} 2-\mathrm{C} 1-\mathrm{C} 7$ & $121.84(14)$ & $\mathrm{H} 8 \mathrm{~B}-\mathrm{C} 8-\mathrm{H} 8 \mathrm{C}$ & 120.5 \\
$\mathrm{C} 6-\mathrm{C} 1-\mathrm{C} 7$ & $119.41(15)$ & $\mathrm{C} 11-\mathrm{C} 10-\mathrm{C} 14$ & 120.5 \\
$\mathrm{C} 3-\mathrm{C} 2-\mathrm{C} 1$ & $120.86(16)$ & $\mathrm{C} 11-\mathrm{C} 10-\mathrm{H} 10$ & $123.73(15)$ \\
$\mathrm{C} 3-\mathrm{C} 2-\mathrm{H} 2$ & 119.6 & $\mathrm{C} 14-\mathrm{C} 10-\mathrm{H} 10$ & \\
$\mathrm{C} 1-\mathrm{C} 2-\mathrm{H} 2$ & 119.6 & $\mathrm{~N} 2-\mathrm{C} 11-\mathrm{C} 10$ &
\end{tabular}




\begin{tabular}{|c|c|c|c|}
\hline $\mathrm{C} 2-\mathrm{C} 3-\mathrm{C} 4$ & $119.69(17)$ & $\mathrm{N} 2-\mathrm{C} 11-\mathrm{H} 11$ & 118.1 \\
\hline $\mathrm{C} 2-\mathrm{C} 3-\mathrm{H} 3$ & 120.2 & $\mathrm{C} 10-\mathrm{C} 11-\mathrm{H} 11$ & 118.1 \\
\hline $\mathrm{C} 4-\mathrm{C} 3-\mathrm{H} 3$ & 120.2 & $\mathrm{~N} 2-\mathrm{C} 12-\mathrm{C} 13$ & $123.55(16)$ \\
\hline $\mathrm{C} 5-\mathrm{C} 4-\mathrm{C} 3$ & $120.01(17)$ & $\mathrm{N} 2-\mathrm{C} 12-\mathrm{H} 12$ & 118.2 \\
\hline $\mathrm{C} 5-\mathrm{C} 4-\mathrm{H} 4$ & 120 & $\mathrm{C} 13-\mathrm{C} 12-\mathrm{H} 12$ & 118.2 \\
\hline $\mathrm{C} 3-\mathrm{C} 4-\mathrm{H} 4$ & 120 & $\mathrm{C} 12-\mathrm{C} 13-\mathrm{C} 14$ & $119.25(14)$ \\
\hline $\mathrm{C} 4-\mathrm{C} 5-\mathrm{C} 6$ & $120.16(17)$ & $\mathrm{C} 12-\mathrm{C} 13-\mathrm{H} 13$ & 120.4 \\
\hline $\mathrm{C} 4-\mathrm{C} 5-\mathrm{H} 5$ & 119.9 & $\mathrm{C} 14-\mathrm{C} 13-\mathrm{H} 13$ & 120.4 \\
\hline $\mathrm{C} 6-\mathrm{C} 5-\mathrm{H} 5$ & 119.9 & $\mathrm{C} 13-\mathrm{C} 14-\mathrm{C} 10$ & $117.66(15)$ \\
\hline $\mathrm{C} 5-\mathrm{C} 6-\mathrm{C} 1$ & $120.53(17)$ & $\mathrm{C} 13-\mathrm{C} 14-\mathrm{C} 15$ & $121.61(13)$ \\
\hline $\mathrm{C} 5-\mathrm{C} 6-\mathrm{H} 6$ & 119.7 & $\mathrm{C} 10-\mathrm{C} 14-\mathrm{C} 15$ & $120.73(14)$ \\
\hline $\mathrm{C} 1-\mathrm{C} 6-\mathrm{H} 6$ & 119.7 & $\mathrm{O} 1-\mathrm{C} 15-\mathrm{O} 2$ & $125.63(15)$ \\
\hline $\mathrm{N} 1-\mathrm{C} 7-\mathrm{C} 1$ & $110.42(13)$ & $\mathrm{O} 1-\mathrm{C} 15-\mathrm{C} 14$ & $117.90(13)$ \\
\hline $\mathrm{N} 1-\mathrm{C} 7-\mathrm{C} 8$ & $109.18(14)$ & $\mathrm{O} 2-\mathrm{C} 15-\mathrm{C} 14$ & $116.46(14)$ \\
\hline $\mathrm{C} 1-\mathrm{C} 7-\mathrm{C} 8$ & $113.54(13)$ & $\mathrm{C} 7-\mathrm{N} 1-\mathrm{H} 1 \mathrm{~A}$ & $110.8(12)$ \\
\hline $\mathrm{N} 1-\mathrm{C} 7-\mathrm{H} 7$ & 107.8 & $\mathrm{C} 7-\mathrm{N} 1-\mathrm{H} 1 \mathrm{~B}$ & $105.6(12)$ \\
\hline $\mathrm{C} 1-\mathrm{C} 7-\mathrm{H} 7$ & 107.8 & $\mathrm{H} 1 \mathrm{~A}-\mathrm{N} 1-\mathrm{H} 1 \mathrm{~B}$ & $115.0(18)$ \\
\hline $\mathrm{C} 8-\mathrm{C} 7-\mathrm{H} 7$ & 107.8 & $\mathrm{C} 7-\mathrm{N} 1-\mathrm{H} 1 \mathrm{C}$ & $110.6(12)$ \\
\hline $\mathrm{C} 7-\mathrm{C} 8-\mathrm{H} 8 \mathrm{~A}$ & 109.5 & $\mathrm{H} 1 \mathrm{~A}-\mathrm{N} 1-\mathrm{H} 1 \mathrm{C}$ & $104.9(16)$ \\
\hline $\mathrm{C} 7-\mathrm{C} 8-\mathrm{H} 8 \mathrm{~B}$ & 109.5 & $\mathrm{H} 1 \mathrm{~B}-\mathrm{N} 1-\mathrm{H} 1 \mathrm{C}$ & $110.0(17)$ \\
\hline $\mathrm{H} 8 \mathrm{~A}-\mathrm{C} 8-\mathrm{H} 8 \mathrm{~B}$ & 109.5 & $\mathrm{C} 11-\mathrm{N} 2-\mathrm{C} 12$ & $116.73(14)$ \\
\hline $\mathrm{C} 7-\mathrm{C} 8-\mathrm{H} 8 \mathrm{C}$ & 109.5 & & \\
\hline $\mathrm{C} 6-\mathrm{C} 1-\mathrm{C} 2-\mathrm{C} 3$ & $-0.7(2)$ & $\mathrm{C} 14-\mathrm{C} 10-\mathrm{C} 11-\mathrm{N} 2$ & $-1.5(3)$ \\
\hline $\mathrm{C} 7-\mathrm{C} 1-\mathrm{C} 2-\mathrm{C} 3$ & $179.00(15)$ & $\mathrm{N} 2-\mathrm{C} 12-\mathrm{C} 13-\mathrm{C} 14$ & $-1.4(3)$ \\
\hline $\mathrm{C} 1-\mathrm{C} 2-\mathrm{C} 3-\mathrm{C} 4$ & $1.4(3)$ & $\mathrm{C} 12-\mathrm{C} 13-\mathrm{C} 14-\mathrm{C} 10$ & $0.4(2)$ \\
\hline $\mathrm{C} 2-\mathrm{C} 3-\mathrm{C} 4-\mathrm{C} 5$ & $-1.1(3)$ & $\mathrm{C} 12-\mathrm{C} 13-\mathrm{C} 14-\mathrm{C} 15$ & $-178.95(14)$ \\
\hline $\mathrm{C} 3-\mathrm{C} 4-\mathrm{C} 5-\mathrm{C} 6$ & $0.2(3)$ & $\mathrm{C} 11-\mathrm{C} 10-\mathrm{C} 14-\mathrm{C} 13$ & $0.9(3)$ \\
\hline $\mathrm{C} 4-\mathrm{C} 5-\mathrm{C} 6-\mathrm{C} 1$ & $0.5(3)$ & $\mathrm{C} 11-\mathrm{C} 10-\mathrm{C} 14-\mathrm{C} 15$ & $-179.69(16)$ \\
\hline $\mathrm{C} 2-\mathrm{C} 1-\mathrm{C} 6-\mathrm{C} 5$ & $-0.2(3)$ & $\mathrm{C} 13-\mathrm{C} 14-\mathrm{C} 15-\mathrm{O} 1$ & $148.63(15)$ \\
\hline $\mathrm{C} 7-\mathrm{C} 1-\mathrm{C} 6-\mathrm{C} 5$ & $-179.94(17)$ & $\mathrm{C} 10-\mathrm{C} 14-\mathrm{C} 15-\mathrm{O} 1$ & $-30.7(2)$ \\
\hline $\mathrm{C} 2-\mathrm{C} 1-\mathrm{C} 7-\mathrm{N} 1$ & $-64.54(19)$ & $\mathrm{C} 13-\mathrm{C} 14-\mathrm{C} 15-\mathrm{O} 2$ & $-30.9(2)$ \\
\hline $\mathrm{C} 6-\mathrm{C} 1-\mathrm{C} 7-\mathrm{N} 1$ & $115.18(17)$ & $\mathrm{C} 10-\mathrm{C} 14-\mathrm{C} 15-\mathrm{O} 2$ & $149.70(16)$ \\
\hline $\mathrm{C} 2-\mathrm{C} 1-\mathrm{C} 7-\mathrm{C} 8$ & $58.5(2)$ & $\mathrm{C} 10-\mathrm{C} 11-\mathrm{N} 2-\mathrm{C} 12$ & $0.6(3)$ \\
\hline $\mathrm{C} 6-\mathrm{C} 1-\mathrm{C} 7-\mathrm{C} 8$ & $-121.83(18)$ & $\mathrm{C} 13-\mathrm{C} 12-\mathrm{N} 2-\mathrm{C} 11$ & $0.9(3)$ \\
\hline
\end{tabular}

Hydrogen-bond geometry $\left(A,{ }^{\circ}\right)$

\begin{tabular}{lllll}
\hline$D-\mathrm{H} \cdots A$ & $D-\mathrm{H}$ & $\mathrm{H} \cdots A$ & $D \cdots A$ & $D-\mathrm{H} \cdots A$ \\
\hline $\mathrm{N} 1-\mathrm{H} 1 B \cdots \mathrm{O} 2^{\mathrm{i}}$ & $0.95(2)$ & $1.80(2)$ & $2.747(2)$ & $176(2)$ \\
$\mathrm{N} 1-\mathrm{H} 1 C \cdots \mathrm{O} 1$ & $0.98(2)$ & $1.81(2)$ & $2.783(2)$ & $174(2)$ \\
$\mathrm{N} 1-\mathrm{H} 1 A \cdots \mathrm{N} 2^{\mathrm{ii}}$ & $0.93(2)$ & $1.93(2)$ & $2.856(2)$ & $175(2)$ \\
\hline
\end{tabular}

Symmetry codes: (i) $-x+3 / 2, y-1 / 2,-z+1 / 2$; (ii) $-x+1,-y+1,-z$. 
(RS)-1-Phenylethan-1-aminium 2-(3-fluoro-4-phenylphenyl)propanoate (II)

Crystal data

$\mathrm{C}_{8} \mathrm{H}_{12} \mathrm{~N}^{+} \cdot \mathrm{C}_{15} \mathrm{H}_{12} \mathrm{FO}_{2}{ }^{-}$
$M_{r}=365.43$
Monoclinic, $P 2_{1} / n$
Hall symbol: $-\mathrm{P} 2 \mathrm{yn}$
$a=12.4146(4) \AA$
$b=5.9101(2) \AA$
$c=27.3645(9) \AA$
$\beta=90.793(1)^{\circ}$
$V=2007.58(11) \AA^{3}$
$Z=4$

Data collection

Bruker D8 Venture Photon CCD area detector diffractometer

Graphite monochromator

$\omega$ scans

Absorption correction: integration

(XPREP; Bruker, 2016)

$T_{\min }=0.984, T_{\max }=0.998$

21119 measured reflections

\section{Refinement}

Refinement on $F^{2}$

Least-squares matrix: full

$R\left[F^{2}>2 \sigma\left(F^{2}\right)\right]=0.061$

$w R\left(F^{2}\right)=0.174$

$S=1.07$

3729 reflections

256 parameters

0 restraints

0 constraints

Special details

Experimental. Numerical integration absorption corrections based on indexed crystal faces were applied using the XPREP routine (Bruker, 2016)

Geometry. All esds (except the esd in the dihedral angle between two 1.s. planes) are estimated using the full covariance matrix. The cell esds are taken into account individually in the estimation of esds in distances, angles and torsion angles; correlations between esds in cell parameters are only used when they are defined by crystal symmetry. An approximate (isotropic) treatment of cell esds is used for estimating esds involving l.s. planes.

Fractional atomic coordinates and isotropic or equivalent isotropic displacement parameters $\left(\AA^{2}\right)$

\begin{tabular}{lllll}
\hline & $x$ & $y$ & $z$ & $U_{\text {iso }} * / U_{\text {eq }}$ \\
\hline $\mathrm{C} 1$ & $1.04935(18)$ & $0.8642(4)$ & $0.27025(8)$ & $0.0329(5)$ \\
$\mathrm{C} 2$ & $1.0760(2)$ & $1.0387(5)$ & $0.30184(9)$ & $0.0417(6)$ \\
$\mathrm{H} 2$ & 1.031358 & 1.168965 & 0.303281 & $0.05^{*}$ \\
$\mathrm{C} 3$ & $1.1675(2)$ & $1.0250(6)$ & $0.33148(10)$ & $0.0530(7)$ \\
$\mathrm{H} 3$ & 1.184766 & 1.1449 & 0.353363 & $0.064^{*}$ \\
$\mathrm{C} 4$ & $1.2332(2)$ & $0.8381(6)$ & $0.32915(12)$ & $0.0601(8)$ \\
$\mathrm{H} 4$ & 1.29587 & 0.828908 & 0.349338 & $0.072^{*}$
\end{tabular}

$F(000)=776$

$D_{\mathrm{x}}=1.209 \mathrm{Mg} \mathrm{m}^{-3}$

Mo $K \alpha$ radiation, $\lambda=0.71073 \AA$

Cell parameters from 8586 reflections

$\theta=3.3-28.1^{\circ}$

$T=173 \mathrm{~K}$

Needle, colourless

$0.49 \times 0.05 \times 0.03 \mathrm{~mm}$

3729 independent reflections

3014 reflections with $I>2 \sigma(I)$

$R_{\text {int }}=0.036$

$\theta_{\max }=25.5^{\circ}, \theta_{\min }=3.0^{\circ}$

$h=-15 \rightarrow 15$

$-7 \rightarrow 7$

Hydrogen site location: mixed

$\mathrm{H}$ atoms treated by a mixture of independent

and constrained refinement

$w=1 /\left[\sigma^{2}\left(F_{\mathrm{o}}^{2}\right)+(0.0822 P)^{2}+1.6935 P\right]$

where $P=\left(F_{\mathrm{o}}^{2}+2 F_{\mathrm{c}}^{2}\right) / 3$

$\Delta \rho_{\max }=1.00 \mathrm{e} \AA^{-3}$
$\Delta \rho_{\min }=-0.29 \mathrm{e} \AA^{-3}$
$(\Delta / \sigma)_{\max }<0.001$ 


\begin{tabular}{|c|c|c|c|c|}
\hline $\mathrm{C} 5$ & $1.2079(2)$ & $0.6647(6)$ & $0.29759(15)$ & $0.0674(9)$ \\
\hline H5 & 1.253609 & 0.536126 & 0.295793 & $0.081^{*}$ \\
\hline C6 & $1.1162(2)$ & $0.6766(5)$ & $0.26840(12)$ & $0.0520(7)$ \\
\hline H6 & 1.098886 & 0.555252 & 0.246917 & $0.062 *$ \\
\hline $\mathrm{C} 7$ & $0.95219(18)$ & $0.8788(4)$ & $0.23622(8)$ & $0.0334(5)$ \\
\hline $\mathrm{H} 7$ & 0.941499 & 0.727244 & 0.220658 & $0.04 *$ \\
\hline $\mathrm{C} 8$ & $0.9665(2)$ & $1.0510(5)$ & $0.19606(9)$ & $0.0466(7)$ \\
\hline $\mathrm{H} 8 \mathrm{~A}$ & 1.031382 & 1.014686 & 0.177604 & $0.07 *$ \\
\hline H8B & 0.90358 & 1.047944 & 0.174065 & $0.07 *$ \\
\hline $\mathrm{H} 8 \mathrm{C}$ & 0.973963 & 1.202164 & 0.210442 & $0.07 *$ \\
\hline C9 & $0.72402(18)$ & $0.2856(4)$ & $0.39881(8)$ & $0.0358(5)$ \\
\hline $\mathrm{C} 10$ & 0.66307 (19) & $0.0970(4)$ & $0.38683(8)$ & $0.0367(5)$ \\
\hline $\mathrm{H} 10$ & 0.694035 & -0.023957 & 0.368967 & $0.044^{*}$ \\
\hline C11 & $0.55715(18)$ & $0.0859(4)$ & $0.40101(8)$ & $0.0329(5)$ \\
\hline $\mathrm{C} 12$ & $0.50615(17)$ & $0.2529(4)$ & $0.42747(7)$ & $0.0284(5)$ \\
\hline $\mathrm{C} 13$ & $0.56906(18)$ & $0.4412(4)$ & $0.43937(8)$ & $0.0327(5)$ \\
\hline H13 & 0.537912 & 0.561242 & 0.457442 & $0.039^{*}$ \\
\hline C14 & $0.67580(19)$ & $0.4579(4)$ & $0.42552(8)$ & $0.0366(5)$ \\
\hline H14 & 0.716474 & 0.588155 & 0.434344 & $0.044 *$ \\
\hline $\mathrm{C} 15$ & $0.39177(17)$ & $0.2360(4)$ & $0.44248(7)$ & $0.0293(5)$ \\
\hline $\mathrm{C} 16$ & $0.35361(19)$ & $0.0448(4)$ & $0.46614(8)$ & $0.0362(5)$ \\
\hline H16 & 0.400371 & -0.079872 & 0.471962 & $0.043^{*}$ \\
\hline $\mathrm{C} 17$ & $0.2475(2)$ & $0.0349(5)$ & $0.48133(9)$ & $0.0437(6)$ \\
\hline H17 & 0.222173 & -0.096227 & 0.497663 & $0.052 *$ \\
\hline $\mathrm{C} 18$ & $0.1787(2)$ & $0.2137(5)$ & $0.47289(9)$ & $0.0471(7)$ \\
\hline $\mathrm{H} 18$ & 0.106092 & 0.206011 & 0.483266 & $0.057^{*}$ \\
\hline C19 & $0.2158(2)$ & $0.4046(5)$ & $0.44923(9)$ & $0.0448(6)$ \\
\hline H19 & 0.168487 & 0.528178 & 0.443247 & $0.054 *$ \\
\hline $\mathrm{C} 20$ & $0.32183(19)$ & $0.4161(4)$ & $0.43423(8)$ & $0.0360(5)$ \\
\hline $\mathrm{H} 20$ & 0.346918 & 0.548087 & 0.418186 & $0.043^{*}$ \\
\hline $\mathrm{C} 21$ & $0.83900(19)$ & $0.3060(5)$ & $0.38057(10)$ & $0.0484(7)$ \\
\hline $\mathrm{H} 21$ & 0.861763 & 0.153443 & 0.368734 & $0.058^{*}$ \\
\hline $\mathrm{C} 22$ & $0.9186(2)$ & $0.3812(8)$ & $0.41767(12)$ & $0.0823(13)$ \\
\hline $\mathrm{H} 22 \mathrm{~A}$ & 0.989972 & 0.389687 & 0.402947 & $0.123^{*}$ \\
\hline $\mathrm{H} 22 \mathrm{~B}$ & 0.898205 & 0.530877 & 0.42989 & $0.123 *$ \\
\hline $\mathrm{H} 22 \mathrm{C}$ & 0.920409 & 0.273085 & 0.44482 & $0.123^{*}$ \\
\hline $\mathrm{C} 23$ & $0.83496(16)$ & $0.4652(4)$ & $0.33647(8)$ & $0.0329(5)$ \\
\hline N1 & $0.85361(16)$ & $0.9339(4)$ & $0.26431(8)$ & 0.0319 (4) \\
\hline $\mathrm{O} 1$ & $0.83181(13)$ & $0.3767(3)$ & $0.29437(6)$ & $0.0388(4)$ \\
\hline $\mathrm{O} 2$ & $0.83086(15)$ & $0.6720(3)$ & $0.34405(6)$ & $0.0470(5)$ \\
\hline $\mathrm{F} 1$ & $0.49883(12)$ & $-0.0979(2)$ & $0.38696(6)$ & 0.0499 \\
\hline $\mathrm{H} 1 \mathrm{~A}$ & $0.797(2)$ & $0.927(5)$ & $0.2430(11)$ & $0.045(7)^{*}$ \\
\hline $\mathrm{H} 1 \mathrm{C}$ & $0.857(2)$ & $1.077(5)$ & $0.2754(10)$ & $0.044(7)^{*}$ \\
\hline H1B & $0.848(2)$ & $0.838(5)$ & $0.2918(11)$ & $0.046(8)^{*}$ \\
\hline
\end{tabular}


Atomic displacement parameters $\left(\AA^{2}\right)$

\begin{tabular}{|c|c|c|c|c|c|c|}
\hline & $U^{11}$ & $U^{22}$ & $U^{33}$ & $U^{12}$ & $U^{13}$ & $U^{23}$ \\
\hline $\mathrm{C} 1$ & $0.0316(11)$ & $0.0356(13)$ & $0.0316(11)$ & $0.0004(10)$ & $0.0037(9)$ & $0.0034(10)$ \\
\hline $\mathrm{C} 2$ & $0.0384(13)$ & $0.0474(15)$ & $0.0391(13)$ & $0.0060(11)$ & $-0.0021(10)$ & $-0.0042(11)$ \\
\hline $\mathrm{C} 3$ & $0.0493(16)$ & $0.069(2)$ & $0.0401(14)$ & $-0.0014(14)$ & $-0.0093(11)$ & $-0.0036(13)$ \\
\hline $\mathrm{C} 4$ & $0.0421(15)$ & $0.074(2)$ & $0.0642(18)$ & $0.0028(15)$ & $-0.0166(13)$ & $0.0232(17)$ \\
\hline $\mathrm{C} 5$ & $0.0456(17)$ & $0.0524(19)$ & $0.104(3)$ & $0.0130(14)$ & $-0.0061(16)$ & $0.0169(18)$ \\
\hline C6 & $0.0447(15)$ & $0.0367(14)$ & $0.0746(19)$ & $0.0029(12)$ & $-0.0002(13)$ & $-0.0020(13)$ \\
\hline $\mathrm{C} 7$ & $0.0342(12)$ & $0.0346(12)$ & $0.0315(11)$ & $-0.0024(10)$ & $0.0025(9)$ & $-0.0048(9)$ \\
\hline $\mathrm{C} 8$ & $0.0420(14)$ & $0.0638(18)$ & $0.0339(12)$ & $-0.0147(13)$ & $-0.0048(10)$ & $0.0077(12)$ \\
\hline C9 & $0.0315(11)$ & $0.0412(13)$ & $0.0346(12)$ & $0.0054(10)$ & $-0.0007(9)$ & $0.0152(10)$ \\
\hline $\mathrm{C} 10$ & $0.0396(13)$ & $0.0340(13)$ & $0.0366(12)$ & $0.0095(10)$ & $0.0060(10)$ & $0.0060(10)$ \\
\hline C11 & $0.0386(12)$ & $0.0277(12)$ & $0.0325(11)$ & $-0.0003(9)$ & $0.0001(9)$ & $0.0020(9)$ \\
\hline $\mathrm{C} 12$ & $0.0331(11)$ & $0.0295(12)$ & $0.0226(10)$ & $0.0023(9)$ & $-0.0021(8)$ & $0.0039(9)$ \\
\hline $\mathrm{C} 13$ & $0.0406(12)$ & $0.0304(12)$ & $0.0269(11)$ & $0.0006(10)$ & $-0.0008(9)$ & $0.0008(9)$ \\
\hline $\mathrm{C} 14$ & $0.0374(12)$ & $0.0378(13)$ & $0.0342(12)$ & $-0.0058(10)$ & $-0.0070(9)$ & $0.0076(10)$ \\
\hline $\mathrm{C} 15$ & $0.0335(11)$ & $0.0318(12)$ & $0.0226(10)$ & $-0.0008(9)$ & $-0.0015(8)$ & $-0.0035(9)$ \\
\hline $\mathrm{C} 16$ & $0.0396(13)$ & $0.0369(13)$ & $0.0323(11)$ & $-0.0005(10)$ & $0.0011(9)$ & $0.0028(10)$ \\
\hline C17 & $0.0434(14)$ & $0.0506(16)$ & $0.0373(13)$ & $-0.0098(12)$ & $0.0033(10)$ & $0.0033(12)$ \\
\hline $\mathrm{C} 18$ & $0.0326(13)$ & $0.0670(19)$ & $0.0418(14)$ & $-0.0058(12)$ & $0.0021(10)$ & $-0.0127(13)$ \\
\hline C19 & $0.0360(13)$ & $0.0499(16)$ & $0.0482(14)$ & $0.0093(11)$ & $-0.0061(11)$ & $-0.0097(12)$ \\
\hline $\mathrm{C} 20$ & $0.0373(12)$ & $0.0341(13)$ & $0.0364(12)$ & $0.0018(10)$ & $-0.0049(9)$ & $-0.0014(10)$ \\
\hline $\mathrm{C} 21$ & $0.0325(13)$ & $0.0591(17)$ & $0.0536(15)$ & $0.0087(12)$ & $0.0033(11)$ & $0.0232(13)$ \\
\hline $\mathrm{C} 22$ & $0.0402(16)$ & $0.150(4)$ & $0.0564(18)$ & $-0.001(2)$ & $-0.0051(14)$ & $0.017(2)$ \\
\hline $\mathrm{C} 23$ & $0.0228(11)$ & $0.0351(13)$ & $0.0409(13)$ & $0.0012(9)$ & $0.0041(9)$ & $0.0070(10)$ \\
\hline N1 & $0.0315(10)$ & $0.0283(11)$ & $0.0358(11)$ & $-0.0018(8)$ & $0.0007(8)$ & $-0.0004(9)$ \\
\hline $\mathrm{O} 1$ & $0.0368(9)$ & $0.0327(9)$ & $0.0467(10)$ & $0.0030(7)$ & $-0.0031(7)$ & $-0.0041(7)$ \\
\hline $\mathrm{O} 2$ & $0.0644(12)$ & $0.0327(10)$ & $0.0445(10)$ & $-0.0038(8)$ & $0.0175(8)$ & $-0.0023(8)$ \\
\hline F1 & $0.0514(9)$ & $0.0378(8)$ & $0.0609(9)$ & $-0.0053(7)$ & $0.0103(7)$ & $-0.0134(7)$ \\
\hline
\end{tabular}

Geometric parameters $\left(\stackrel{A}{\circ}{ }^{\circ}\right)$

\begin{tabular}{llll}
\hline $\mathrm{C} 1-\mathrm{C} 2$ & $1.383(3)$ & $\mathrm{C} 12-\mathrm{C} 15$ & $1.487(3)$ \\
$\mathrm{C} 1-\mathrm{C} 6$ & $1.386(4)$ & $\mathrm{C} 13-\mathrm{C} 14$ & $1.387(3)$ \\
$\mathrm{C} 1-\mathrm{C} 7$ & $1.516(3)$ & $\mathrm{C} 13-\mathrm{H} 13$ & 0.95 \\
$\mathrm{C} 2-\mathrm{C} 3$ & $1.389(4)$ & $\mathrm{C} 14-\mathrm{H} 14$ & 0.95 \\
$\mathrm{C} 2-\mathrm{H} 2$ & 0.95 & $\mathrm{C} 15-\mathrm{C} 16$ & $1.389(3)$ \\
$\mathrm{C} 3-\mathrm{C} 4$ & $1.375(4)$ & $\mathrm{C} 15-\mathrm{C} 20$ & $1.390(3)$ \\
$\mathrm{C} 3-\mathrm{H} 3$ & 0.95 & $\mathrm{C} 16-\mathrm{C} 17$ & $1.388(3)$ \\
$\mathrm{C} 4-\mathrm{C} 5$ & $1.374(5)$ & $\mathrm{C} 16-\mathrm{H} 16$ & 0.95 \\
$\mathrm{C} 4-\mathrm{H} 4$ & 0.95 & $\mathrm{C} 17-\mathrm{C} 18$ & $1.376(4)$ \\
$\mathrm{C} 5-\mathrm{C} 6$ & $1.384(4)$ & $\mathrm{C} 17-\mathrm{H} 17$ & 0.95 \\
$\mathrm{C} 5-\mathrm{H} 5$ & 0.95 & $\mathrm{C} 18-\mathrm{C} 19$ & $1.383(4)$ \\
$\mathrm{C} 6-\mathrm{H} 6$ & 0.95 & $\mathrm{C} 18-\mathrm{H} 18$ & 0.95 \\
$\mathrm{C} 7-\mathrm{N} 1$ & $1.490(3)$ & $\mathrm{C} 19-\mathrm{C} 20$ & $1.386(3)$ \\
$\mathrm{C} 7-\mathrm{C} 8$ & $1.510(3)$ & $\mathrm{C} 19-\mathrm{H} 19$ & 0.95 \\
$\mathrm{C} 7-\mathrm{H} 7$ & 1 & $\mathrm{C} 20-\mathrm{H} 20$ & 0.95
\end{tabular}




\begin{tabular}{|c|c|c|c|}
\hline $\mathrm{C} 8-\mathrm{H} 8 \mathrm{~A}$ & 0.98 & $\mathrm{C} 21-\mathrm{C} 22$ & $1.475(4)$ \\
\hline $\mathrm{C} 8-\mathrm{H} 8 \mathrm{~B}$ & 0.98 & $\mathrm{C} 21-\mathrm{C} 23$ & $1.531(3)$ \\
\hline $\mathrm{C} 8-\mathrm{H} 8 \mathrm{C}$ & 0.98 & $\mathrm{C} 21-\mathrm{H} 21$ & 1 \\
\hline $\mathrm{C} 9-\mathrm{C} 10$ & $1.384(3)$ & $\mathrm{C} 22-\mathrm{H} 22 \mathrm{~A}$ & 0.98 \\
\hline C9-C14 & $1.394(4)$ & $\mathrm{C} 22-\mathrm{H} 22 \mathrm{~B}$ & 0.98 \\
\hline $\mathrm{C} 9-\mathrm{C} 21$ & $1.523(3)$ & $\mathrm{C} 22-\mathrm{H} 22 \mathrm{C}$ & 0.98 \\
\hline $\mathrm{C} 10-\mathrm{C} 11$ & $1.378(3)$ & $\mathrm{C} 23-\mathrm{O} 2$ & $1.241(3)$ \\
\hline $\mathrm{C} 10-\mathrm{H} 10$ & 0.95 & $\mathrm{C} 23-\mathrm{O} 1$ & $1.265(3)$ \\
\hline $\mathrm{C} 11-\mathrm{F} 1$ & $1.358(3)$ & $\mathrm{N} 1-\mathrm{H} 1 \mathrm{~A}$ & $0.91(3)$ \\
\hline $\mathrm{C} 11-\mathrm{C} 12$ & $1.383(3)$ & $\mathrm{N} 1-\mathrm{H} 1 \mathrm{C}$ & $0.90(3)$ \\
\hline $\mathrm{C} 12-\mathrm{C} 13$ & $1.396(3)$ & $\mathrm{N} 1-\mathrm{H} 1 \mathrm{~B}$ & $0.95(3)$ \\
\hline $\mathrm{C} 2-\mathrm{C} 1-\mathrm{C} 6$ & $118.8(2)$ & $\mathrm{C} 12-\mathrm{C} 13-\mathrm{H} 13$ & 119.1 \\
\hline $\mathrm{C} 2-\mathrm{C} 1-\mathrm{C} 7$ & $121.5(2)$ & $\mathrm{C} 13-\mathrm{C} 14-\mathrm{C} 9$ & $120.7(2)$ \\
\hline $\mathrm{C} 6-\mathrm{C} 1-\mathrm{C} 7$ & $119.7(2)$ & $\mathrm{C} 13-\mathrm{C} 14-\mathrm{H} 14$ & 119.7 \\
\hline $\mathrm{C} 1-\mathrm{C} 2-\mathrm{C} 3$ & $120.6(2)$ & $\mathrm{C} 9-\mathrm{C} 14-\mathrm{H} 14$ & 119.7 \\
\hline $\mathrm{C} 1-\mathrm{C} 2-\mathrm{H} 2$ & 119.7 & $\mathrm{C} 16-\mathrm{C} 15-\mathrm{C} 20$ & $118.8(2)$ \\
\hline $\mathrm{C} 3-\mathrm{C} 2-\mathrm{H} 2$ & 119.7 & $\mathrm{C} 16-\mathrm{C} 15-\mathrm{C} 12$ & $121.1(2)$ \\
\hline $\mathrm{C} 4-\mathrm{C} 3-\mathrm{C} 2$ & $120.0(3)$ & $\mathrm{C} 20-\mathrm{C} 15-\mathrm{C} 12$ & $120.0(2)$ \\
\hline $\mathrm{C} 4-\mathrm{C} 3-\mathrm{H} 3$ & 120 & $\mathrm{C} 17-\mathrm{C} 16-\mathrm{C} 15$ & $120.3(2)$ \\
\hline $\mathrm{C} 2-\mathrm{C} 3-\mathrm{H} 3$ & 120 & $\mathrm{C} 17-\mathrm{C} 16-\mathrm{H} 16$ & 119.8 \\
\hline $\mathrm{C} 5-\mathrm{C} 4-\mathrm{C} 3$ & $119.9(3)$ & $\mathrm{C} 15-\mathrm{C} 16-\mathrm{H} 16$ & 119.8 \\
\hline $\mathrm{C} 5-\mathrm{C} 4-\mathrm{H} 4$ & 120 & $\mathrm{C} 18-\mathrm{C} 17-\mathrm{C} 16$ & $120.4(2)$ \\
\hline $\mathrm{C} 3-\mathrm{C} 4-\mathrm{H} 4$ & 120 & $\mathrm{C} 18-\mathrm{C} 17-\mathrm{H} 17$ & 119.8 \\
\hline $\mathrm{C} 4-\mathrm{C} 5-\mathrm{C} 6$ & $120.2(3)$ & $\mathrm{C} 16-\mathrm{C} 17-\mathrm{H} 17$ & 119.8 \\
\hline $\mathrm{C} 4-\mathrm{C} 5-\mathrm{H} 5$ & 119.9 & $\mathrm{C} 17-\mathrm{C} 18-\mathrm{C} 19$ & $119.7(2)$ \\
\hline $\mathrm{C} 6-\mathrm{C} 5-\mathrm{H} 5$ & 119.9 & $\mathrm{C} 17-\mathrm{C} 18-\mathrm{H} 18$ & 120.2 \\
\hline $\mathrm{C} 5-\mathrm{C} 6-\mathrm{C} 1$ & $120.5(3)$ & $\mathrm{C} 19-\mathrm{C} 18-\mathrm{H} 18$ & 120.2 \\
\hline $\mathrm{C} 5-\mathrm{C} 6-\mathrm{H} 6$ & 119.7 & $\mathrm{C} 18-\mathrm{C} 19-\mathrm{C} 20$ & $120.1(2)$ \\
\hline $\mathrm{C} 1-\mathrm{C} 6-\mathrm{H} 6$ & 119.7 & $\mathrm{C} 18-\mathrm{C} 19-\mathrm{H} 19$ & 119.9 \\
\hline $\mathrm{N} 1-\mathrm{C} 7-\mathrm{C} 8$ & $109.5(2)$ & $\mathrm{C} 20-\mathrm{C} 19-\mathrm{H} 19$ & 119.9 \\
\hline $\mathrm{N} 1-\mathrm{C} 7-\mathrm{C} 1$ & $110.34(18)$ & $\mathrm{C} 19-\mathrm{C} 20-\mathrm{C} 15$ & $120.5(2)$ \\
\hline $\mathrm{C} 8-\mathrm{C} 7-\mathrm{C} 1$ & $112.63(19)$ & $\mathrm{C} 19-\mathrm{C} 20-\mathrm{H} 20$ & 119.7 \\
\hline $\mathrm{N} 1-\mathrm{C} 7-\mathrm{H} 7$ & 108.1 & $\mathrm{C} 15-\mathrm{C} 20-\mathrm{H} 20$ & 119.7 \\
\hline $\mathrm{C} 8-\mathrm{C} 7-\mathrm{H} 7$ & 108.1 & $\mathrm{C} 22-\mathrm{C} 21-\mathrm{C} 9$ & $114.8(2)$ \\
\hline $\mathrm{C} 1-\mathrm{C} 7-\mathrm{H} 7$ & 108.1 & $\mathrm{C} 22-\mathrm{C} 21-\mathrm{C} 23$ & $111.8(3)$ \\
\hline $\mathrm{C} 7-\mathrm{C} 8-\mathrm{H} 8 \mathrm{~A}$ & 109.5 & $\mathrm{C} 9-\mathrm{C} 21-\mathrm{C} 23$ & $106.63(18)$ \\
\hline $\mathrm{C} 7-\mathrm{C} 8-\mathrm{H} 8 \mathrm{~B}$ & 109.5 & $\mathrm{C} 22-\mathrm{C} 21-\mathrm{H} 21$ & 107.8 \\
\hline $\mathrm{H} 8 \mathrm{~A}-\mathrm{C} 8-\mathrm{H} 8 \mathrm{~B}$ & 109.5 & $\mathrm{C} 9-\mathrm{C} 21-\mathrm{H} 21$ & 107.8 \\
\hline $\mathrm{C} 7-\mathrm{C} 8-\mathrm{H} 8 \mathrm{C}$ & 109.5 & $\mathrm{C} 23-\mathrm{C} 21-\mathrm{H} 21$ & 107.8 \\
\hline $\mathrm{H} 8 \mathrm{~A}-\mathrm{C} 8-\mathrm{H} 8 \mathrm{C}$ & 109.5 & $\mathrm{C} 21-\mathrm{C} 22-\mathrm{H} 22 \mathrm{~A}$ & 109.5 \\
\hline $\mathrm{H} 8 \mathrm{~B}-\mathrm{C} 8-\mathrm{H} 8 \mathrm{C}$ & 109.5 & $\mathrm{C} 21-\mathrm{C} 22-\mathrm{H} 22 \mathrm{~B}$ & 109.5 \\
\hline $\mathrm{C} 10-\mathrm{C} 9-\mathrm{C} 14$ & $118.4(2)$ & $\mathrm{H} 22 \mathrm{~A}-\mathrm{C} 22-\mathrm{H} 22 \mathrm{~B}$ & 109.5 \\
\hline $\mathrm{C} 10-\mathrm{C} 9-\mathrm{C} 21$ & $119.8(2)$ & $\mathrm{C} 21-\mathrm{C} 22-\mathrm{H} 22 \mathrm{C}$ & 109.5 \\
\hline $\mathrm{C} 14-\mathrm{C} 9-\mathrm{C} 21$ & $121.7(2)$ & $\mathrm{H} 22 \mathrm{~A}-\mathrm{C} 22-\mathrm{H} 22 \mathrm{C}$ & 109.5 \\
\hline $\mathrm{C} 11-\mathrm{C} 10-\mathrm{C} 9$ & $119.5(2)$ & $\mathrm{H} 22 \mathrm{~B}-\mathrm{C} 22-\mathrm{H} 22 \mathrm{C}$ & 109.5 \\
\hline $\mathrm{C} 11-\mathrm{C} 10-\mathrm{H} 10$ & 120.3 & $\mathrm{O} 2-\mathrm{C} 23-\mathrm{O} 1$ & $124.0(2)$ \\
\hline $\mathrm{C} 9-\mathrm{C} 10-\mathrm{H} 10$ & 120.3 & $\mathrm{O} 2-\mathrm{C} 23-\mathrm{C} 21$ & $118.3(2)$ \\
\hline
\end{tabular}




\begin{tabular}{|c|c|c|c|}
\hline $\mathrm{F} 1-\mathrm{C} 11-\mathrm{C} 10$ & $117.7(2)$ & $\mathrm{O} 1-\mathrm{C} 23-\mathrm{C} 21$ & $117.6(2)$ \\
\hline $\mathrm{F} 1-\mathrm{C} 11-\mathrm{C} 12$ & $118.2(2)$ & $\mathrm{C} 7-\mathrm{N} 1-\mathrm{H} 1 \mathrm{~A}$ & $107.0(17)$ \\
\hline $\mathrm{C} 10-\mathrm{C} 11-\mathrm{C} 12$ & $124.0(2)$ & $\mathrm{C} 7-\mathrm{N} 1-\mathrm{H} 1 \mathrm{C}$ & $110.5(18)$ \\
\hline $\mathrm{C} 11-\mathrm{C} 12-\mathrm{C} 13$ & $115.6(2)$ & $\mathrm{H} 1 \mathrm{~A}-\mathrm{N} 1-\mathrm{H} 1 \mathrm{C}$ & $107(2)$ \\
\hline $\mathrm{C} 11-\mathrm{C} 12-\mathrm{C} 15$ & $122.9(2)$ & $\mathrm{C} 7-\mathrm{N} 1-\mathrm{H} 1 \mathrm{~B}$ & $110.3(17)$ \\
\hline $\mathrm{C} 13-\mathrm{C} 12-\mathrm{C} 15$ & $121.50(19)$ & $\mathrm{H} 1 \mathrm{~A}-\mathrm{N} 1-\mathrm{H} 1 \mathrm{~B}$ & $115(2)$ \\
\hline $\mathrm{C} 14-\mathrm{C} 13-\mathrm{C} 12$ & $121.8(2)$ & $\mathrm{H} 1 \mathrm{C}-\mathrm{N} 1-\mathrm{H} 1 \mathrm{~B}$ & $108(2)$ \\
\hline $\mathrm{C} 14-\mathrm{C} 13-\mathrm{H} 13$ & 119.1 & & \\
\hline $\mathrm{C} 6-\mathrm{C} 1-\mathrm{C} 2-\mathrm{C} 3$ & $-0.6(4)$ & $\mathrm{C} 10-\mathrm{C} 9-\mathrm{C} 14-\mathrm{C} 13$ & $-0.5(3)$ \\
\hline $\mathrm{C} 7-\mathrm{C} 1-\mathrm{C} 2-\mathrm{C} 3$ & $-177.7(2)$ & $\mathrm{C} 21-\mathrm{C} 9-\mathrm{C} 14-\mathrm{C} 13$ & $176.3(2)$ \\
\hline $\mathrm{C} 1-\mathrm{C} 2-\mathrm{C} 3-\mathrm{C} 4$ & $0.8(4)$ & $\mathrm{C} 11-\mathrm{C} 12-\mathrm{C} 15-\mathrm{C} 16$ & $50.7(3)$ \\
\hline $\mathrm{C} 2-\mathrm{C} 3-\mathrm{C} 4-\mathrm{C} 5$ & $-0.2(5)$ & $\mathrm{C} 13-\mathrm{C} 12-\mathrm{C} 15-\mathrm{C} 16$ & $-129.4(2)$ \\
\hline $\mathrm{C} 3-\mathrm{C} 4-\mathrm{C} 5-\mathrm{C} 6$ & $-0.5(5)$ & $\mathrm{C} 11-\mathrm{C} 12-\mathrm{C} 15-\mathrm{C} 20$ & $-131.3(2)$ \\
\hline $\mathrm{C} 4-\mathrm{C} 5-\mathrm{C} 6-\mathrm{C} 1$ & $0.7(5)$ & $\mathrm{C} 13-\mathrm{C} 12-\mathrm{C} 15-\mathrm{C} 20$ & $48.6(3)$ \\
\hline $\mathrm{C} 2-\mathrm{C} 1-\mathrm{C} 6-\mathrm{C} 5$ & $-0.1(4)$ & $\mathrm{C} 20-\mathrm{C} 15-\mathrm{C} 16-\mathrm{C} 17$ & $-0.2(3)$ \\
\hline $\mathrm{C} 7-\mathrm{C} 1-\mathrm{C} 6-\mathrm{C} 5$ & $177.1(3)$ & $\mathrm{C} 12-\mathrm{C} 15-\mathrm{C} 16-\mathrm{C} 17$ & $177.9(2)$ \\
\hline $\mathrm{C} 2-\mathrm{C} 1-\mathrm{C} 7-\mathrm{N} 1$ & $-53.5(3)$ & $\mathrm{C} 15-\mathrm{C} 16-\mathrm{C} 17-\mathrm{C} 18$ & $0.4(4)$ \\
\hline $\mathrm{C} 6-\mathrm{C} 1-\mathrm{C} 7-\mathrm{N} 1$ & $129.5(2)$ & $\mathrm{C} 16-\mathrm{C} 17-\mathrm{C} 18-\mathrm{C} 19$ & $-0.2(4)$ \\
\hline $\mathrm{C} 2-\mathrm{C} 1-\mathrm{C} 7-\mathrm{C} 8$ & $69.3(3)$ & $\mathrm{C} 17-\mathrm{C} 18-\mathrm{C} 19-\mathrm{C} 20$ & $-0.2(4)$ \\
\hline $\mathrm{C} 6-\mathrm{C} 1-\mathrm{C} 7-\mathrm{C} 8$ & $-107.8(3)$ & $\mathrm{C} 18-\mathrm{C} 19-\mathrm{C} 20-\mathrm{C} 15$ & $0.4(4)$ \\
\hline $\mathrm{C} 14-\mathrm{C} 9-\mathrm{C} 10-\mathrm{C} 11$ & $0.7(3)$ & $\mathrm{C} 16-\mathrm{C} 15-\mathrm{C} 20-\mathrm{C} 19$ & $-0.2(3)$ \\
\hline $\mathrm{C} 21-\mathrm{C} 9-\mathrm{C} 10-\mathrm{C} 11$ & $-176.2(2)$ & $\mathrm{C} 12-\mathrm{C} 15-\mathrm{C} 20-\mathrm{C} 19$ & $-178.3(2)$ \\
\hline $\mathrm{C} 9-\mathrm{C} 10-\mathrm{C} 11-\mathrm{F} 1$ & $177.5(2)$ & $\mathrm{C} 10-\mathrm{C} 9-\mathrm{C} 21-\mathrm{C} 22$ & $-134.9(3)$ \\
\hline $\mathrm{C} 9-\mathrm{C} 10-\mathrm{C} 11-\mathrm{C} 12$ & $-0.7(3)$ & $\mathrm{C} 14-\mathrm{C} 9-\mathrm{C} 21-\mathrm{C} 22$ & $48.3(4)$ \\
\hline $\mathrm{F} 1-\mathrm{C} 11-\mathrm{C} 12-\mathrm{C} 13$ & $-177.81(18)$ & $\mathrm{C} 10-\mathrm{C} 9-\mathrm{C} 21-\mathrm{C} 23$ & $100.7(3)$ \\
\hline $\mathrm{C} 10-\mathrm{C} 11-\mathrm{C} 12-\mathrm{C} 13$ & $0.4(3)$ & $\mathrm{C} 14-\mathrm{C} 9-\mathrm{C} 21-\mathrm{C} 23$ & $-76.1(3)$ \\
\hline $\mathrm{F} 1-\mathrm{C} 11-\mathrm{C} 12-\mathrm{C} 15$ & $2.1(3)$ & $\mathrm{C} 22-\mathrm{C} 21-\mathrm{C} 23-\mathrm{O} 2$ & $-46.8(3)$ \\
\hline $\mathrm{C} 10-\mathrm{C} 11-\mathrm{C} 12-\mathrm{C} 15$ & $-179.7(2)$ & $\mathrm{C} 9-\mathrm{C} 21-\mathrm{C} 23-\mathrm{O} 2$ & $79.5(3)$ \\
\hline $\mathrm{C} 11-\mathrm{C} 12-\mathrm{C} 13-\mathrm{C} 14$ & $-0.2(3)$ & $\mathrm{C} 22-\mathrm{C} 21-\mathrm{C} 23-\mathrm{O} 1$ & $135.9(3)$ \\
\hline $\mathrm{C} 15-\mathrm{C} 12-\mathrm{C} 13-\mathrm{C} 14$ & $179.93(19)$ & $\mathrm{C} 9-\mathrm{C} 21-\mathrm{C} 23-\mathrm{O} 1$ & $-97.8(3)$ \\
\hline $\mathrm{C} 12-\mathrm{C} 13-\mathrm{C} 14-\mathrm{C} 9$ & $0.3(3)$ & & \\
\hline
\end{tabular}

Hydrogen-bond geometry $\left(\AA,{ }^{\circ}\right)$

\begin{tabular}{lllll}
\hline$D-\mathrm{H}^{\cdots} A$ & $D-\mathrm{H}$ & $\mathrm{H} \cdots A$ & $D \cdots A$ & $D-\mathrm{H}^{\cdots} A$ \\
\hline $\mathrm{N} 1-\mathrm{H} 1 A^{\cdots} \mathrm{O} 1^{\mathrm{i}}$ & $0.91(3)$ & $1.91(3)$ & $2.809(3)$ & $170(3)$ \\
$\mathrm{N} 1-\mathrm{H} 1 C \cdots \mathrm{O} 1^{i i}$ & $0.90(3)$ & $1.87(3)$ & $2.758(3)$ & $168(3)$ \\
$\mathrm{N} 1-\mathrm{H} 1 B \cdots \mathrm{O} 2$ & $0.95(3)$ & $1.75(3)$ & $2.693(3)$ & $176(3)$ \\
\hline
\end{tabular}

Symmetry codes: (i) $-x+3 / 2, y+1 / 2,-z+1 / 2$; (ii) $x, y+1, z$.

(RS)-1-Phenylethan-1-aminium 2-chloro-4-nitrobenzoate (III)

Crystal data

$\mathrm{C}_{8} \mathrm{H}_{12} \mathrm{~N}^{+} \cdot \mathrm{C}_{7} \mathrm{H}_{3} \mathrm{ClNO}_{4}^{-}$

$M_{r}=322.74$

Monoclinic, $C 2 / c$

Hall symbol: -C $2 \mathrm{yc}$

$$
\begin{aligned}
& a=15.5817(7) \AA \\
& b=6.3914(3) \AA \\
& c=31.3238(14) \AA \\
& \beta=100.998(2)^{\circ}
\end{aligned}
$$




$$
\begin{aligned}
& V=3062.2(2) \AA^{3} \\
& Z=8 \\
& F(000)=1344 \\
& D_{\mathrm{x}}=1.4 \mathrm{Mg} \mathrm{m}^{-3}
\end{aligned}
$$

Mo $K \alpha$ radiation, $\lambda=0.71073 \AA$

Cell parameters from 8180 reflections

\section{Data collection}

Bruker D8 Venture Photon CCD area detector diffractometer

Graphite monochromator

$\omega$ scans

Absorption correction: integration

(XPREP; Bruker, 2016)

$T_{\min }=0.903, T_{\max }=0.979$

18885 measured reflections

\section{Refinement}

Refinement on $F^{2}$

Least-squares matrix: full

$R\left[F^{2}>2 \sigma\left(F^{2}\right)\right]=0.035$

$w R\left(F^{2}\right)=0.091$

$S=1.04$

3709 reflections

212 parameters

0 restraints

0 constraints

$$
\begin{aligned}
& \theta=2.7-28.3^{\circ} \\
& \mu=0.27 \mathrm{~mm}^{-1} \\
& T=173 \mathrm{~K} \\
& \text { Plate, colourless } \\
& 0.47 \times 0.35 \times 0.08 \mathrm{~mm}
\end{aligned}
$$

3709 independent reflections 3218 reflections with $I>2 \sigma(I)$

$R_{\text {int }}=0.053$

$\theta_{\max }=28.0^{\circ}, \theta_{\min }=2.7^{\circ}$

$h=-20 \rightarrow 20$

$k=-8 \rightarrow 8$

$l=-41 \rightarrow 41$

\section{Special details}

Experimental. Numerical integration absorption corrections based on indexed crystal faces were applied using the XPREP routine (Bruker, 2016)

Geometry. All esds (except the esd in the dihedral angle between two 1.s. planes) are estimated using the full covariance matrix. The cell esds are taken into account individually in the estimation of esds in distances, angles and torsion angles; correlations between esds in cell parameters are only used when they are defined by crystal symmetry. An approximate (isotropic) treatment of cell esds is used for estimating esds involving l.s. planes.

Fractional atomic coordinates and isotropic or equivalent isotropic displacement parameters $\left(\AA^{2}\right)$

\begin{tabular}{lllll}
\hline & $x$ & $y$ & $z$ & $U_{\text {iso }}^{*} / U_{\text {eq }}$ \\
\hline C1 & $0.09581(10)$ & $0.3921(3)$ & $0.05256(5)$ & $0.0435(4)$ \\
H1 & 0.088661 & 0.371465 & 0.022052 & $0.052^{*}$ \\
C2 & $0.09009(10)$ & $0.2257(3)$ & $0.07959(5)$ & $0.0440(4)$ \\
H2 & 0.079856 & 0.089053 & 0.067772 & $0.053^{*}$ \\
C3 & $0.09920(9)$ & $0.2562(2)$ & $0.12412(5)$ & $0.0360(3)$ \\
H3 & 0.094958 & 0.140054 & 0.142536 & $0.043^{*}$ \\
C4 & $0.11437(8)$ & $0.4538(2)$ & $0.14197(4)$ & $0.0263(3)$ \\
C5 & $0.12131(11)$ & $0.6209(2)$ & $0.11467(5)$ & $0.0412(3)$ \\
H5 & 0.132454 & 0.757299 & 0.126471 & $0.049^{*}$ \\
C6 & $0.11206(12)$ & $0.5899(3)$ & $0.07006(5)$ & $0.0492(4)$ \\
H6 & 0.116926 & 0.705265 & 0.051546 & $0.059^{*}$ \\
C7 & $0.12507(8)$ & $0.4829(2)$ & $0.19075(4)$ & $0.0261(3)$ \\
H7 & 0.108406 & 0.348778 & 0.20347 & $0.031^{*}$ \\
C8 & $0.06945(9)$ & $0.6569(2)$ & $0.20441(4)$ & $0.0353(3)$
\end{tabular}




$\begin{array}{lllll}\text { H8A } & 0.007558 & 0.627874 & 0.193002 & 0.053^{*} \\ \text { H8B } & 0.085549 & 0.790563 & 0.192793 & 0.053^{*} \\ \text { H8C } & 0.079383 & 0.664261 & 0.236219 & 0.053^{*} \\ \text { N1 } & 0.21931(6) & 0.52603(17) & 0.20964(3) & 0.0233(2) \\ \text { H1A } & 0.2343(10) & 0.661(3) & 0.2022(5) & 0.035(4)^{*} \\ \text { H1B } & 0.2564(10) & 0.429(3) & 0.1998(5) & 0.034(4)^{*} \\ \text { H1C } & 0.2276(10) & 0.518(2) & 0.2403(5) & 0.033(4)^{*} \\ \text { C9 } & 0.33113(8) & 0.90589(19) & 0.14294(4) & 0.0244(2) \\ \text { C10 } & 0.37797(7) & 0.72139(19) & 0.15321(4) & 0.0236(2) \\ \text { C11 } & 0.39367(8) & 0.5834(2) & 0.12153(4) & 0.0277(3) \\ \text { H11 } & 0.423888 & 0.455476 & 0.128894 & 0.033^{*} \\ \text { C12 } & 0.36346(9) & 0.6399(2) & 0.07865(4) & 0.0330(3) \\ \text { C13 } & 0.32140(11) & 0.8263(2) & 0.06657(4) & 0.0386(3) \\ \text { H13 } & 0.303972 & 0.862985 & 0.03679 & 0.046^{*} \\ \text { C14 } & 0.30526(9) & 0.9589(2) & 0.09922(4) & 0.0332(3) \\ \text { H14 } & 0.276081 & 1.087962 & 0.091646 & 0.04^{*} \\ \text { C15 } & 0.30282(7) & 1.04387(19) & 0.17704(4) & 0.0238(2) \\ \text { N2 } & 0.37830(10) & 0.4929(2) & 0.04459(4) & 0.0453(3) \\ \text { O1 } & 0.25740(6) & 0.95511(14) & 0.20132(3) & 0.0280(2) \\ \text { O2 } & 0.32294(6) & 1.23110(14) & 0.17769(3) & 0.0323(2) \\ \text { O3 } & 0.41721(10) & 0.33069(19) & 0.05555(4) & 0.0595(4) \\ \text { O4 } & 0.35101(12) & 0.5415(2) & 0.00671(4) & 0.0796(5) \\ \text { C11 } & 0.42338(2) & 0.66402(5) & 0.20708(2) & 0.02886(10) \\ & & & & \end{array}$

Atomic displacement parameters $\left(\AA^{2}\right)$

\begin{tabular}{lllllll}
\hline & $U^{11}$ & $U^{22}$ & $U^{33}$ & $U^{12}$ & $U^{13}$ & $U^{23}$ \\
\hline C1 & $0.0479(8)$ & $0.0530(10)$ & $0.0283(6)$ & $0.0043(7)$ & $0.0041(6)$ & $-0.0096(6)$ \\
C2 & $0.0523(9)$ & $0.0377(9)$ & $0.0410(8)$ & $-0.0035(7)$ & $0.0058(7)$ & $-0.0159(7)$ \\
C3 & $0.0414(7)$ & $0.0285(7)$ & $0.0380(7)$ & $-0.0027(6)$ & $0.0071(6)$ & $-0.0027(6)$ \\
C4 & $0.0260(5)$ & $0.0264(7)$ & $0.0264(6)$ & $-0.0023(5)$ & $0.0051(4)$ & $-0.0016(5)$ \\
C5 & $0.0664(10)$ & $0.0279(7)$ & $0.0295(7)$ & $-0.0040(7)$ & $0.0095(6)$ & $-0.0011(5)$ \\
C6 & $0.0767(11)$ & $0.0428(9)$ & $0.0283(7)$ & $0.0003(8)$ & $0.0106(7)$ & $0.0048(6)$ \\
C7 & $0.0276(5)$ & $0.0258(6)$ & $0.0260(6)$ & $-0.0049(5)$ & $0.0078(4)$ & $0.0010(5)$ \\
C8 & $0.0308(6)$ & $0.0439(9)$ & $0.0334(7)$ & $0.0035(6)$ & $0.0113(5)$ & $-0.0021(6)$ \\
N1 & $0.0283(5)$ & $0.0208(5)$ & $0.0221(5)$ & $-0.0008(4)$ & $0.0080(4)$ & $-0.0002(4)$ \\
C9 & $0.0294(6)$ & $0.0209(6)$ & $0.0244(5)$ & $-0.0018(4)$ & $0.0093(4)$ & $-0.0007(4)$ \\
C10 & $0.0267(5)$ & $0.0246(6)$ & $0.0200(5)$ & $-0.0031(4)$ & $0.0059(4)$ & $0.0000(4)$ \\
C11 & $0.0331(6)$ & $0.0240(6)$ & $0.0275(6)$ & $0.0020(5)$ & $0.0097(5)$ & $-0.0007(5)$ \\
C12 & $0.0474(7)$ & $0.0300(7)$ & $0.0247(6)$ & $0.0032(6)$ & $0.0145(5)$ & $-0.0030(5)$ \\
C13 & $0.0591(9)$ & $0.0362(8)$ & $0.0222(6)$ & $0.0069(6)$ & $0.0119(6)$ & $0.0040(5)$ \\
C14 & $0.0482(7)$ & $0.0268(7)$ & $0.0267(6)$ & $0.0073(6)$ & $0.0121(5)$ & $0.0052(5)$ \\
C15 & $0.0272(5)$ & $0.0223(6)$ & $0.0220(5)$ & $0.0013(4)$ & $0.0052(4)$ & $-0.0004(4)$ \\
N2 & $0.0727(9)$ & $0.0388(8)$ & $0.0279(6)$ & $0.0080(6)$ & $0.0186(6)$ & $-0.0049(5)$ \\
O1 & $0.0368(5)$ & $0.0249(5)$ & $0.0251(4)$ & $-0.0034(4)$ & $0.0127(3)$ & $-0.0024(3)$ \\
O2 & $0.0407(5)$ & $0.0201(5)$ & $0.0396(5)$ & $-0.0024(4)$ & $0.0167(4)$ & $-0.0030(4)$ \\
O3 & $0.0975(10)$ & $0.0428(7)$ & $0.0406(6)$ & $0.0242(7)$ & $0.0192(6)$ & $-0.0079(5)$ \\
O4 & $0.1472(14)$ & $0.0678(9)$ & $0.0243(5)$ & $0.0380(9)$ & $0.0182(7)$ & $-0.0036(6)$
\end{tabular}


Geometric parameters $(\AA, \stackrel{o}{)}$

\begin{tabular}{|c|c|c|c|}
\hline $\mathrm{C} 1-\mathrm{C} 2$ & $1.373(2)$ & $\mathrm{N} 1-\mathrm{H} 1 \mathrm{~B}$ & $0.940(16)$ \\
\hline $\mathrm{C} 1-\mathrm{C} 6$ & $1.382(2)$ & $\mathrm{N} 1-\mathrm{H} 1 \mathrm{C}$ & $0.946(16)$ \\
\hline $\mathrm{C} 1-\mathrm{H} 1$ & 0.95 & $\mathrm{C} 9-\mathrm{C} 10$ & $1.3919(17)$ \\
\hline $\mathrm{C} 2-\mathrm{C} 3$ & $1.389(2)$ & $\mathrm{C} 9-\mathrm{C} 14$ & $1.3933(17)$ \\
\hline $\mathrm{C} 2-\mathrm{H} 2$ & 0.95 & $\mathrm{C} 9-\mathrm{C} 15$ & $1.5143(16)$ \\
\hline $\mathrm{C} 3-\mathrm{C} 4$ & $1.3833(19)$ & $\mathrm{C} 10-\mathrm{C} 11$ & $1.3837(17)$ \\
\hline $\mathrm{C} 3-\mathrm{H} 3$ & 0.95 & $\mathrm{C} 10-\mathrm{Cl1}$ & $1.7397(11)$ \\
\hline $\mathrm{C} 4-\mathrm{C} 5$ & $1.3848(19)$ & $\mathrm{C} 11-\mathrm{C} 12$ & $1.3836(18)$ \\
\hline $\mathrm{C} 4-\mathrm{C} 7$ & $1.5167(16)$ & $\mathrm{C} 11-\mathrm{H} 11$ & 0.95 \\
\hline $\mathrm{C} 5-\mathrm{C} 6$ & $1.391(2)$ & $\mathrm{C} 12-\mathrm{C} 13$ & $1.377(2)$ \\
\hline $\mathrm{C} 5-\mathrm{H} 5$ & 0.95 & $\mathrm{C} 12-\mathrm{N} 2$ & $1.4726(17)$ \\
\hline $\mathrm{C} 6-\mathrm{H} 6$ & 0.95 & $\mathrm{C} 13-\mathrm{C} 14$ & $1.3876(19)$ \\
\hline $\mathrm{C} 7-\mathrm{N} 1$ & $1.5003(15)$ & $\mathrm{C} 13-\mathrm{H} 13$ & 0.95 \\
\hline $\mathrm{C} 7-\mathrm{C} 8$ & $1.5206(19)$ & $\mathrm{C} 14-\mathrm{H} 14$ & 0.95 \\
\hline $\mathrm{C} 7-\mathrm{H} 7$ & 1 & $\mathrm{C} 15-\mathrm{O} 2$ & $1.2362(15)$ \\
\hline $\mathrm{C} 8-\mathrm{H} 8 \mathrm{~A}$ & 0.98 & $\mathrm{C} 15-\mathrm{O} 1$ & $1.2675(14)$ \\
\hline $\mathrm{C} 8-\mathrm{H} 8 \mathrm{~B}$ & 0.98 & $\mathrm{~N} 2-\mathrm{O} 3$ & $1.2171(17)$ \\
\hline $\mathrm{C} 8-\mathrm{H} 8 \mathrm{C}$ & 0.98 & $\mathrm{~N} 2-\mathrm{O} 4$ & $1.2216(17)$ \\
\hline $\mathrm{N} 1-\mathrm{H} 1 \mathrm{~A}$ & $0.937(17)$ & & \\
\hline $\mathrm{C} 2-\mathrm{C} 1-\mathrm{C} 6$ & $119.52(13)$ & $\mathrm{C} 7-\mathrm{N} 1-\mathrm{H} 1 \mathrm{~A}$ & $110.0(9)$ \\
\hline $\mathrm{C} 2-\mathrm{C} 1-\mathrm{H} 1$ & 120.2 & $\mathrm{C} 7-\mathrm{N} 1-\mathrm{H} 1 \mathrm{~B}$ & $111.4(9)$ \\
\hline $\mathrm{C} 6-\mathrm{C} 1-\mathrm{H} 1$ & 120.2 & $\mathrm{H} 1 \mathrm{~A}-\mathrm{N} 1-\mathrm{H} 1 \mathrm{~B}$ & $109.4(13)$ \\
\hline $\mathrm{C} 1-\mathrm{C} 2-\mathrm{C} 3$ & $120.29(14)$ & $\mathrm{C} 7-\mathrm{N} 1-\mathrm{H} 1 \mathrm{C}$ & $108.9(9)$ \\
\hline $\mathrm{C} 1-\mathrm{C} 2-\mathrm{H} 2$ & 119.9 & $\mathrm{H} 1 \mathrm{~A}-\mathrm{N} 1-\mathrm{H} 1 \mathrm{C}$ & $107.9(13)$ \\
\hline $\mathrm{C} 3-\mathrm{C} 2-\mathrm{H} 2$ & 119.9 & $\mathrm{H} 1 \mathrm{~B}-\mathrm{N} 1-\mathrm{H} 1 \mathrm{C}$ & $109.0(13)$ \\
\hline $\mathrm{C} 4-\mathrm{C} 3-\mathrm{C} 2$ & $120.72(14)$ & $\mathrm{C} 10-\mathrm{C} 9-\mathrm{C} 14$ & $118.28(11)$ \\
\hline $\mathrm{C} 4-\mathrm{C} 3-\mathrm{H} 3$ & 119.6 & $\mathrm{C} 10-\mathrm{C} 9-\mathrm{C} 15$ & $122.74(10)$ \\
\hline $\mathrm{C} 2-\mathrm{C} 3-\mathrm{H} 3$ & 119.6 & $\mathrm{C} 14-\mathrm{C} 9-\mathrm{C} 15$ & $118.89(11)$ \\
\hline $\mathrm{C} 3-\mathrm{C} 4-\mathrm{C} 5$ & $118.82(12)$ & $\mathrm{C} 11-\mathrm{C} 10-\mathrm{C} 9$ & $122.05(11)$ \\
\hline $\mathrm{C} 3-\mathrm{C} 4-\mathrm{C} 7$ & $119.75(12)$ & $\mathrm{C} 11-\mathrm{C} 10-\mathrm{C} 11$ & $117.81(9)$ \\
\hline $\mathrm{C} 5-\mathrm{C} 4-\mathrm{C} 7$ & $121.41(12)$ & $\mathrm{C} 9-\mathrm{C} 10-\mathrm{Cl} 1$ & $120.07(9)$ \\
\hline $\mathrm{C} 4-\mathrm{C} 5-\mathrm{C} 6$ & $120.33(14)$ & $\mathrm{C} 12-\mathrm{C} 11-\mathrm{C} 10$ & $117.13(12)$ \\
\hline $\mathrm{C} 4-\mathrm{C} 5-\mathrm{H} 5$ & 119.8 & $\mathrm{C} 12-\mathrm{C} 11-\mathrm{H} 11$ & 121.4 \\
\hline $\mathrm{C} 6-\mathrm{C} 5-\mathrm{H} 5$ & 119.8 & $\mathrm{C} 10-\mathrm{C} 11-\mathrm{H} 11$ & 121.4 \\
\hline $\mathrm{C} 1-\mathrm{C} 6-\mathrm{C} 5$ & $120.30(15)$ & $\mathrm{C} 13-\mathrm{C} 12-\mathrm{C} 11$ & $123.24(12)$ \\
\hline $\mathrm{C} 1-\mathrm{C} 6-\mathrm{H} 6$ & 119.8 & $\mathrm{C} 13-\mathrm{C} 12-\mathrm{N} 2$ & $119.02(12)$ \\
\hline $\mathrm{C} 5-\mathrm{C} 6-\mathrm{H} 6$ & 119.8 & $\mathrm{C} 11-\mathrm{C} 12-\mathrm{N} 2$ & $117.74(12)$ \\
\hline $\mathrm{N} 1-\mathrm{C} 7-\mathrm{C} 4$ & $109.33(9)$ & $\mathrm{C} 12-\mathrm{C} 13-\mathrm{C} 14$ & $118.00(12)$ \\
\hline $\mathrm{N} 1-\mathrm{C} 7-\mathrm{C} 8$ & $108.79(10)$ & $\mathrm{C} 12-\mathrm{C} 13-\mathrm{H} 13$ & 121 \\
\hline $\mathrm{C} 4-\mathrm{C} 7-\mathrm{C} 8$ & $114.45(11)$ & $\mathrm{C} 14-\mathrm{C} 13-\mathrm{H} 13$ & 121 \\
\hline $\mathrm{N} 1-\mathrm{C} 7-\mathrm{H} 7$ & 108 & $\mathrm{C} 13-\mathrm{C} 14-\mathrm{C} 9$ & $121.11(12)$ \\
\hline $\mathrm{C} 4-\mathrm{C} 7-\mathrm{H} 7$ & 108 & $\mathrm{C} 13-\mathrm{C} 14-\mathrm{H} 14$ & 119.4 \\
\hline $\mathrm{C} 8-\mathrm{C} 7-\mathrm{H} 7$ & 108 & $\mathrm{C} 9-\mathrm{C} 14-\mathrm{H} 14$ & 119.4 \\
\hline
\end{tabular}




$\begin{array}{llll}\mathrm{C} 7-\mathrm{C} 8-\mathrm{H} 8 \mathrm{~A} & 109.5 & \mathrm{O} 2-\mathrm{C} 15-\mathrm{O} 1 & 126.32(11) \\ \mathrm{C} 7-\mathrm{C} 8-\mathrm{H} 8 \mathrm{~B} & 109.5 & \mathrm{O} 2-\mathrm{C} 15-\mathrm{C} 9 & 117.92(10) \\ \mathrm{H} 8 \mathrm{~A}-\mathrm{C} 8-\mathrm{H} 8 \mathrm{~B} & 109.5 & \mathrm{O} 1-\mathrm{C} 15-\mathrm{C} 9 & 115.71(11) \\ \mathrm{C} 7-\mathrm{C} 8-\mathrm{H} 8 \mathrm{C} & 109.5 & \mathrm{O} 3-\mathrm{N} 2-\mathrm{O} 4 & 123.54(13) \\ \mathrm{H} 8 \mathrm{~A}-\mathrm{C} 8-\mathrm{H} 8 \mathrm{C} & 109.5 & \mathrm{O} 3-\mathrm{N} 2-\mathrm{C} 12 & 117.55(12) \\ \mathrm{H} 8 \mathrm{~B}-\mathrm{C} 8-\mathrm{H} 8 \mathrm{C} & 109.5 & \mathrm{O} 4-\mathrm{N} 2-\mathrm{C} 12 & -174.67(13) \\ & & & 1.7(2) \\ \mathrm{C} 6-\mathrm{C} 1-\mathrm{C} 2-\mathrm{C} 3 & 1.0(2) & \mathrm{C} 11-\mathrm{C} 10-\mathrm{C} 11-\mathrm{C} 12 & -178.93(12) \\ \mathrm{C} 1-\mathrm{C} 2-\mathrm{C} 3-\mathrm{C} 4 & -0.2(2) & \mathrm{C} 10-\mathrm{C} 11-\mathrm{C} 12-\mathrm{C} 13 & -3.0(2) \\ \mathrm{C} 2-\mathrm{C} 3-\mathrm{C} 4-\mathrm{C} 5 & -0.7(2) & \mathrm{C} 10-\mathrm{C} 11-\mathrm{C} 12-\mathrm{N} 2 & 177.66(14) \\ \mathrm{C} 2-\mathrm{C} 3-\mathrm{C} 4-\mathrm{C} 7 & -179.33(13) & \mathrm{C} 11-\mathrm{C} 12-\mathrm{C} 13-\mathrm{C} 14 & 0.3(2) \\ \mathrm{C} 3-\mathrm{C} 4-\mathrm{C} 5-\mathrm{C} 6 & 0.8(2) & \mathrm{N} 2-\mathrm{C} 12-\mathrm{C} 13-\mathrm{C} 14 & 3.5(2) \\ \mathrm{C} 7-\mathrm{C} 4-\mathrm{C} 5-\mathrm{C} 6 & 179.39(14) & \mathrm{C} 12-\mathrm{C} 13-\mathrm{C} 14-\mathrm{C} 9 & -173.03(13) \\ \mathrm{C} 2-\mathrm{C} 1-\mathrm{C} 6-\mathrm{C} 5 & -0.9(3) & \mathrm{C} 10-\mathrm{C} 9-\mathrm{C} 14-\mathrm{C} 13 & 126.58(13) \\ \mathrm{C} 4-\mathrm{C} 5-\mathrm{C} 6-\mathrm{C} 1 & 0.0(3) & \mathrm{C} 15-\mathrm{C} 9-\mathrm{C} 14-\mathrm{C} 13 & -57.09(16) \\ \mathrm{C} 3-\mathrm{C} 4-\mathrm{C} 7-\mathrm{N} 1 & 107.18(13) & \mathrm{C} 10-\mathrm{C} 9-\mathrm{C} 15-\mathrm{O} 2 & -55.96(15) \\ \mathrm{C} 5-\mathrm{C} 4-\mathrm{C} 7-\mathrm{N} 1 & -71.41(16) & \mathrm{C} 14-\mathrm{C} 9-\mathrm{C} 15-\mathrm{O} 2 & 120.37(13) \\ \mathrm{C} 3-\mathrm{C} 4-\mathrm{C} 7-\mathrm{C} 8 & -130.51(13) & \mathrm{C} 10-\mathrm{C} 9-\mathrm{C} 15-\mathrm{O} 1 & 178.42(16) \\ \mathrm{C} 5-\mathrm{C} 4-\mathrm{C} 7-\mathrm{C} 8 & 50.90(17) & \mathrm{C} 14-\mathrm{C} 9-\mathrm{C} 15-\mathrm{O} 1 & -0.9(2) \\ \mathrm{C} 14-\mathrm{C} 9-\mathrm{C} 10-\mathrm{C} 11 & -4.84(18) & \mathrm{C} 13-\mathrm{C} 12-\mathrm{N} 2-\mathrm{O} 3 & -1.3(2) \\ \mathrm{C} 15-\mathrm{C} 9-\mathrm{C} 10-\mathrm{C} 11 & 171.52(11) & \mathrm{C} 11-\mathrm{C} 12-\mathrm{N} 2-\mathrm{O} 3 & 179.34(16) \\ \mathrm{C} 14-\mathrm{C} 9-\mathrm{C} 10-\mathrm{C} 11 & 172.07(10) & \mathrm{C} 13-\mathrm{C} 12-\mathrm{N} 2-\mathrm{O} 4 & \\ \mathrm{C} 15-\mathrm{C} 9-\mathrm{C} 10-\mathrm{C} 11 & -11.58(16) & \mathrm{C} 11-\mathrm{C} 12-\mathrm{N} 2-\mathrm{O} 4 & \\ \mathrm{C} 9-\mathrm{C} 10-\mathrm{C} 11-\mathrm{C} 12 & 2.30(18) & & \end{array}$

Hydrogen-bond geometry $\left(\AA,{ }^{\circ}\right)$

\begin{tabular}{lllll}
\hline$D-\mathrm{H} \cdots A$ & $D-\mathrm{H}$ & $\mathrm{H} \cdots A$ & $D \cdots A$ & $D-\mathrm{H}^{\cdots} A$ \\
\hline $\mathrm{N} 1-\mathrm{H} 1 A \cdots \mathrm{O} 1$ & $0.94(2)$ & $1.91(2)$ & $2.829(1)$ & $165(1)$ \\
$\mathrm{N} 1-\mathrm{H} 1 B \cdots \mathrm{O} 2^{\mathrm{i}}$ & $0.94(2)$ & $1.85(2)$ & $2.789(1)$ & $176(1)$ \\
$\mathrm{N} 1-\mathrm{H} 1 C \cdots \mathrm{O} 1^{\text {ii }}$ & $0.95(2)$ & $1.84(2)$ & $2.780(1)$ & $170(1)$ \\
\hline
\end{tabular}

Symmetry codes: (i) $x, y-1, z$; (ii) $-x+1 / 2, y-1 / 2,-z+1 / 2$.

(RS)-1-Phenylethan-1-aminium 4-iodobenzoate (IV)

\section{Crystal data}

$\mathrm{C}_{8} \mathrm{H}_{12} \mathrm{~N}^{+} \cdot \mathrm{C}_{7} \mathrm{H}_{4} \mathrm{IO}_{2}^{-}$

$M_{r}=369.19$

Monoclinic, $P 2_{1} / n$

Hall symbol: -P 2 yn

$a=9.7224(5) \AA$

$b=6.0571(3) \AA$

$c=24.8767$ (12) $\AA$

$\beta=99.527(2)^{\circ}$

$V=1444.77(12) \AA^{3}$

$Z=4$
$F(000)=728$

$D_{\mathrm{x}}=1.697 \mathrm{Mg} \mathrm{m}^{-3}$

Mo $K \alpha$ radiation, $\lambda=0.71073 \AA$

Cell parameters from 9122 reflections

$\theta=3.3-28.3^{\circ}$

$\mu=2.21 \mathrm{~mm}^{-1}$

$T=173 \mathrm{~K}$

Plate, orange

$0.68 \times 0.16 \times 0.04 \mathrm{~mm}$ 


\section{Data collection}

Bruker D8 Venture Photon CCD area detector diffractometer

Graphite monochromator

$\omega$ scans

Absorption correction: integration

(XPREP; Bruker, 2016)

$T_{\min }=0.508, T_{\max }=0.928$

30978 measured reflections

\section{Refinement}

Refinement on $F^{2}$

Least-squares matrix: full

$R\left[F^{2}>2 \sigma\left(F^{2}\right)\right]=0.026$

$w R\left(F^{2}\right)=0.057$

$S=1.13$

3463 reflections

185 parameters

0 restraints

0 constraints
3463 independent reflections

3109 reflections with $I>2 \sigma(I)$

$R_{\text {int }}=0.051$

$\theta_{\max }=28.0^{\circ}, \theta_{\min }=3.0^{\circ}$

$h=-12 \rightarrow 12$

$k=-8 \rightarrow 8$

$l=-32 \rightarrow 32$

\section{Special details}

Experimental. Numerical integration absorption corrections based on indexed crystal faces were applied using the XPREP routine (Bruker, 2016)

Geometry. All esds (except the esd in the dihedral angle between two 1.s. planes) are estimated using the full covariance matrix. The cell esds are taken into account individually in the estimation of esds in distances, angles and torsion angles; correlations between esds in cell parameters are only used when they are defined by crystal symmetry. An approximate (isotropic) treatment of cell esds is used for estimating esds involving 1.s. planes.

Fractional atomic coordinates and isotropic or equivalent isotropic displacement parameters $\left(\hat{A}^{2}\right)$

\begin{tabular}{lllll}
\hline & $x$ & $y$ & $z$ & $U_{\text {iso }} * / U_{\text {eq }}$ \\
\hline C1 & $0.4776(2)$ & $0.5625(4)$ & $0.30540(9)$ & $0.0228(4)$ \\
$\mathrm{C} 2$ & $0.5527(2)$ & $0.3668(4)$ & $0.30591(9)$ & $0.0264(5)$ \\
H2 & 0.600702 & 0.334421 & 0.276565 & $0.032^{*}$ \\
$\mathrm{C} 3$ & $0.5584(2)$ & $0.2182(4)$ & $0.34868(10)$ & $0.0273(5)$ \\
$\mathrm{H} 3$ & 0.611349 & 0.086327 & 0.348888 & $0.033^{*}$ \\
$\mathrm{C} 4$ & $0.4864(2)$ & $0.2631(4)$ & $0.39109(10)$ & $0.0277(5)$ \\
$\mathrm{H} 4$ & 0.488952 & 0.161314 & 0.420293 & $0.033^{*}$ \\
$\mathrm{C} 5$ & $0.4111(2)$ & $0.4563(4)$ & $0.39071(9)$ & $0.0292(5)$ \\
$\mathrm{H} 5$ & 0.361284 & 0.486833 & 0.419662 & $0.035^{*}$ \\
$\mathrm{C} 6$ & $0.4075(2)$ & $0.6059(4)$ & $0.34849(9)$ & $0.0273(5)$ \\
$\mathrm{H} 6$ & 0.356522 & 0.739462 & 0.348997 & $0.033^{*}$ \\
$\mathrm{C} 7$ & $0.4644(2)$ & $0.7258(4)$ & $0.25903(10)$ & $0.0263(5)$ \\
$\mathrm{H} 7$ & 0.440241 & 0.872568 & 0.273366 & $0.032^{*}$ \\
$\mathrm{C} 8$ & $0.3496(3)$ & $0.6640(5)$ & $0.21243(11)$ & $0.0361(6)$ \\
$\mathrm{H} 8 \mathrm{~A}$ & 0.366585 & 0.515084 & 0.199602 & $0.054^{*}$ \\
$\mathrm{H} 8 \mathrm{~B}$ & 0.348559 & 0.769447 & 0.182486 & $0.054^{*}$ \\
$\mathrm{H} 8 \mathrm{C}$ & 0.259354 & 0.667562 & 0.225099 & $0.054^{*}$ \\
$\mathrm{~N} 1$ & $0.5992(2)$ & $0.7512(3)$ & $0.23771(8)$ & $0.0214(4)$ \\
$\mathrm{H} 1 \mathrm{~A}$ & $0.621(3)$ & $0.633(5)$ & $0.2208(10)$ & $0.023(6)^{*}$
\end{tabular}




$\begin{array}{lllll}\text { H1B } & 0.591(3) & 0.858(5) & 0.2159(11) & 0.026(7)^{*} \\ \text { H1C } & 0.670(3) & 0.780(4) & 0.2662(12) & 0.031(7)^{*} \\ \text { C9 } & 0.5029(2) & 0.3948(4) & 0.10780(8) & 0.0204(4) \\ \text { C10 } & 0.5399(2) & 0.6044(4) & 0.09238(9) & 0.0233(4) \\ \text { H10 } & 0.623639 & 0.670573 & 0.110297 & 0.028^{*} \\ \text { C11 } & 0.4551(2) & 0.7177(4) & 0.05091(9) & 0.0249(4) \\ \text { H11 } & 0.480966 & 0.860353 & 0.040232 & 0.03^{*} \\ \text { C12 } & 0.3327(2) & 0.6210(4) & 0.02534(8) & 0.0240(4) \\ \text { C13 } & 0.2946(2) & 0.4109(4) & 0.03976(9) & 0.0279(5) \\ \text { H13 } & 0.210789 & 0.344932 & 0.021782 & 0.034^{*} \\ \text { C14 } & 0.3806(2) & 0.2995(4) & 0.08068(9) & 0.0246(4) \\ \text { H14 } & 0.355719 & 0.155055 & 0.0905 & 0.029^{*} \\ \text { C15 } & 0.5894(2) & 0.2737(4) & 0.15462(9) & 0.0220(4) \\ \text { O1 } & 0.68527(16) & 0.3835(3) & 0.18395(6) & 0.0249(3) \\ \text { O2 } & 0.5591(2) & 0.0792(3) & 0.16235(7) & 0.0375(4) \\ \text { I1 } & 0.19967(2) & 0.79494(3) & -0.03513(2) & 0.03359(6)\end{array}$

Atomic displacement parameters $\left(\AA^{2}\right)$

\begin{tabular}{lllllll}
\hline & $U^{11}$ & $U^{22}$ & $U^{33}$ & $U^{12}$ & $U^{13}$ & $U^{23}$ \\
\hline C1 & $0.0175(10)$ & $0.0242(11)$ & $0.0258(11)$ & $-0.0025(8)$ & $0.0005(8)$ & $0.0018(9)$ \\
C2 & $0.0263(11)$ & $0.0296(12)$ & $0.0245(11)$ & $0.0037(9)$ & $0.0076(9)$ & $0.0029(9)$ \\
C3 & $0.0279(11)$ & $0.0245(11)$ & $0.0292(12)$ & $0.0029(9)$ & $0.0039(9)$ & $0.0025(9)$ \\
C4 & $0.0262(11)$ & $0.0326(13)$ & $0.0239(11)$ & $-0.0014(9)$ & $0.0029(9)$ & $0.0042(9)$ \\
C5 & $0.0254(11)$ & $0.0395(14)$ & $0.0236(11)$ & $0.0012(10)$ & $0.0063(9)$ & $-0.0019(10)$ \\
C6 & $0.0226(11)$ & $0.0278(12)$ & $0.0308(12)$ & $0.0048(9)$ & $0.0023(9)$ & $-0.0002(10)$ \\
C7 & $0.0219(10)$ & $0.0269(11)$ & $0.0300(12)$ & $0.0042(9)$ & $0.0036(9)$ & $0.0039(9)$ \\
C8 & $0.0250(12)$ & $0.0472(16)$ & $0.0339(13)$ & $0.0017(11)$ & $-0.0019(10)$ & $0.0057(12)$ \\
N1 & $0.0213(9)$ & $0.0191(10)$ & $0.0224(9)$ & $-0.0012(7)$ & $-0.0009(7)$ & $0.0037(8)$ \\
C9 & $0.0229(10)$ & $0.0205(10)$ & $0.0183(9)$ & $0.0012(8)$ & $0.0046(8)$ & $0.0004(8)$ \\
C10 & $0.0251(11)$ & $0.0214(11)$ & $0.0224(10)$ & $-0.0032(9)$ & $0.0010(8)$ & $0.0002(9)$ \\
C11 & $0.0330(12)$ & $0.0201(10)$ & $0.0219(10)$ & $0.0008(9)$ & $0.0056(9)$ & $0.0036(9)$ \\
C12 & $0.0290(11)$ & $0.0242(11)$ & $0.0178(10)$ & $0.0077(9)$ & $0.0010(8)$ & $-0.0010(9)$ \\
C13 & $0.0263(11)$ & $0.0264(12)$ & $0.0289(12)$ & $-0.0016(9)$ & $-0.0017(9)$ & $-0.0023(10)$ \\
C14 & $0.0264(11)$ & $0.0193(10)$ & $0.0275(11)$ & $-0.0030(9)$ & $0.0031(9)$ & $0.0017(9)$ \\
C15 & $0.0258(10)$ & $0.0216(10)$ & $0.0189(10)$ & $0.0019(8)$ & $0.0047(8)$ & $0.0020(8)$ \\
O1 & $0.0251(8)$ & $0.0259(8)$ & $0.0218(7)$ & $0.0011(6)$ & $-0.0020(6)$ & $0.0004(6)$ \\
O2 & $0.0508(11)$ & $0.0234(9)$ & $0.0334(9)$ & $-0.0059(8)$ & $-0.0069(8)$ & $0.0109(7)$ \\
I1 & $0.03935(10)$ & $0.03639(10)$ & $0.02241(9)$ & $0.01375(7)$ & $-0.00260(6)$ & $0.00224(6)$ \\
& & & & & &
\end{tabular}

Geometric parameters $\left(\AA,{ }^{\circ}\right)$

\begin{tabular}{llll}
\hline $\mathrm{C} 1-\mathrm{C} 6$ & $1.387(3)$ & $\mathrm{N} 1-\mathrm{H} 1 \mathrm{~A}$ & $0.88(3)$ \\
$\mathrm{C} 1-\mathrm{C} 2$ & $1.391(3)$ & $\mathrm{N} 1-\mathrm{H} 1 \mathrm{~B}$ & $0.84(3)$ \\
$\mathrm{C} 1-\mathrm{C} 7$ & $1.509(3)$ & $\mathrm{N} 1-\mathrm{H} 1 \mathrm{C}$ & $0.92(3)$ \\
$\mathrm{C} 2-\mathrm{C} 3$ & $1.388(3)$ & $\mathrm{C} 9-\mathrm{C} 10$ & $1.390(3)$ \\
$\mathrm{C} 2-\mathrm{H} 2$ & 0.95 & $\mathrm{C} 9-\mathrm{C} 14$ & $1.392(3)$ \\
$\mathrm{C} 3-\mathrm{C} 4$ & $1.386(3)$ & $\mathrm{C} 9-\mathrm{C} 15$ & $1.509(3)$
\end{tabular}




\begin{tabular}{|c|c|c|c|}
\hline $\mathrm{C} 3-\mathrm{H} 3$ & 0.95 & $\mathrm{C} 10-\mathrm{C} 11$ & $1.391(3)$ \\
\hline $\mathrm{C} 4-\mathrm{C} 5$ & $1.380(3)$ & $\mathrm{C} 10-\mathrm{H} 10$ & 0.95 \\
\hline $\mathrm{C} 4-\mathrm{H} 4$ & 0.95 & $\mathrm{C} 11-\mathrm{C} 12$ & $1.383(3)$ \\
\hline $\mathrm{C} 5-\mathrm{C} 6$ & $1.383(3)$ & $\mathrm{C} 11-\mathrm{H} 11$ & 0.95 \\
\hline $\mathrm{C} 5-\mathrm{H} 5$ & 0.95 & $\mathrm{C} 12-\mathrm{C} 13$ & $1.389(3)$ \\
\hline $\mathrm{C} 6-\mathrm{H} 6$ & 0.95 & $\mathrm{C} 12-\mathrm{I} 1$ & $2.098(2)$ \\
\hline $\mathrm{C} 7-\mathrm{N} 1$ & $1.501(3)$ & $\mathrm{C} 13-\mathrm{C} 14$ & $1.382(3)$ \\
\hline $\mathrm{C} 7-\mathrm{C} 8$ & $1.517(3)$ & $\mathrm{C} 13-\mathrm{H} 13$ & 0.95 \\
\hline $\mathrm{C} 7-\mathrm{H} 7$ & 1 & $\mathrm{C} 14-\mathrm{H} 14$ & 0.95 \\
\hline $\mathrm{C} 8-\mathrm{H} 8 \mathrm{~A}$ & 0.98 & $\mathrm{C} 15-\mathrm{O} 2$ & $1.237(3)$ \\
\hline $\mathrm{C} 8-\mathrm{H} 8 \mathrm{~B}$ & 0.98 & $\mathrm{C} 15-\mathrm{O} 1$ & $1.272(3)$ \\
\hline $\mathrm{C} 8-\mathrm{H} 8 \mathrm{C}$ & 0.98 & & \\
\hline $\mathrm{C} 6-\mathrm{C} 1-\mathrm{C} 2$ & $118.6(2)$ & $\mathrm{H} 8 \mathrm{~B}-\mathrm{C} 8-\mathrm{H} 8 \mathrm{C}$ & 109.5 \\
\hline $\mathrm{C} 6-\mathrm{C} 1-\mathrm{C} 7$ & $118.4(2)$ & $\mathrm{C} 7-\mathrm{N} 1-\mathrm{H} 1 \mathrm{~A}$ & $112.5(17)$ \\
\hline $\mathrm{C} 2-\mathrm{C} 1-\mathrm{C} 7$ & $123.0(2)$ & $\mathrm{C} 7-\mathrm{N} 1-\mathrm{H} 1 \mathrm{~B}$ & $108.3(18)$ \\
\hline $\mathrm{C} 3-\mathrm{C} 2-\mathrm{C} 1$ & $120.9(2)$ & $\mathrm{H} 1 \mathrm{~A}-\mathrm{N} 1-\mathrm{H} 1 \mathrm{~B}$ & $109(2)$ \\
\hline $\mathrm{C} 3-\mathrm{C} 2-\mathrm{H} 2$ & 119.5 & $\mathrm{C} 7-\mathrm{N} 1-\mathrm{H} 1 \mathrm{C}$ & $109.4(17)$ \\
\hline $\mathrm{C} 1-\mathrm{C} 2-\mathrm{H} 2$ & 119.5 & $\mathrm{H} 1 \mathrm{~A}-\mathrm{N} 1-\mathrm{H} 1 \mathrm{C}$ & $108(2)$ \\
\hline $\mathrm{C} 4-\mathrm{C} 3-\mathrm{C} 2$ & $119.7(2)$ & $\mathrm{H} 1 \mathrm{~B}-\mathrm{N} 1-\mathrm{H} 1 \mathrm{C}$ & $110(2)$ \\
\hline $\mathrm{C} 4-\mathrm{C} 3-\mathrm{H} 3$ & 120.2 & $\mathrm{C} 10-\mathrm{C} 9-\mathrm{C} 14$ & $118.9(2)$ \\
\hline $\mathrm{C} 2-\mathrm{C} 3-\mathrm{H} 3$ & 120.2 & $\mathrm{C} 10-\mathrm{C} 9-\mathrm{C} 15$ & $121.37(19)$ \\
\hline $\mathrm{C} 5-\mathrm{C} 4-\mathrm{C} 3$ & $119.7(2)$ & $\mathrm{C} 14-\mathrm{C} 9-\mathrm{C} 15$ & $119.64(19)$ \\
\hline $\mathrm{C} 5-\mathrm{C} 4-\mathrm{H} 4$ & 120.1 & $\mathrm{C} 9-\mathrm{C} 10-\mathrm{C} 11$ & $120.5(2)$ \\
\hline $\mathrm{C} 3-\mathrm{C} 4-\mathrm{H} 4$ & 120.1 & $\mathrm{C} 9-\mathrm{C} 10-\mathrm{H} 10$ & 119.8 \\
\hline $\mathrm{C} 4-\mathrm{C} 5-\mathrm{C} 6$ & $120.5(2)$ & $\mathrm{C} 11-\mathrm{C} 10-\mathrm{H} 10$ & 119.8 \\
\hline $\mathrm{C} 4-\mathrm{C} 5-\mathrm{H} 5$ & 119.8 & $\mathrm{C} 12-\mathrm{C} 11-\mathrm{C} 10$ & $119.4(2)$ \\
\hline $\mathrm{C} 6-\mathrm{C} 5-\mathrm{H} 5$ & 119.8 & $\mathrm{C} 12-\mathrm{C} 11-\mathrm{H} 11$ & 120.3 \\
\hline $\mathrm{C} 5-\mathrm{C} 6-\mathrm{C} 1$ & $120.6(2)$ & $\mathrm{C} 10-\mathrm{C} 11-\mathrm{H} 11$ & 120.3 \\
\hline $\mathrm{C} 5-\mathrm{C} 6-\mathrm{H} 6$ & 119.7 & $\mathrm{C} 11-\mathrm{C} 12-\mathrm{C} 13$ & $121.0(2)$ \\
\hline $\mathrm{C} 1-\mathrm{C} 6-\mathrm{H} 6$ & 119.7 & $\mathrm{C} 11-\mathrm{C} 12-\mathrm{I} 1$ & $119.89(17)$ \\
\hline $\mathrm{N} 1-\mathrm{C} 7-\mathrm{C} 1$ & $111.61(18)$ & $\mathrm{C} 13-\mathrm{C} 12-\mathrm{I} 1$ & $119.08(16)$ \\
\hline $\mathrm{N} 1-\mathrm{C} 7-\mathrm{C} 8$ & $109.3(2)$ & $\mathrm{C} 14-\mathrm{C} 13-\mathrm{C} 12$ & $118.9(2)$ \\
\hline $\mathrm{C} 1-\mathrm{C} 7-\mathrm{C} 8$ & $112.4(2)$ & $\mathrm{C} 14-\mathrm{C} 13-\mathrm{H} 13$ & 120.6 \\
\hline $\mathrm{N} 1-\mathrm{C} 7-\mathrm{H} 7$ & 107.8 & $\mathrm{C} 12-\mathrm{C} 13-\mathrm{H} 13$ & 120.6 \\
\hline $\mathrm{C} 1-\mathrm{C} 7-\mathrm{H} 7$ & 107.8 & $\mathrm{C} 13-\mathrm{C} 14-\mathrm{C} 9$ & $121.2(2)$ \\
\hline $\mathrm{C} 8-\mathrm{C} 7-\mathrm{H} 7$ & 107.8 & $\mathrm{C} 13-\mathrm{C} 14-\mathrm{H} 14$ & 119.4 \\
\hline $\mathrm{C} 7-\mathrm{C} 8-\mathrm{H} 8 \mathrm{~A}$ & 109.5 & $\mathrm{C} 9-\mathrm{C} 14-\mathrm{H} 14$ & 119.4 \\
\hline $\mathrm{C} 7-\mathrm{C} 8-\mathrm{H} 8 \mathrm{~B}$ & 109.5 & $\mathrm{O} 2-\mathrm{C} 15-\mathrm{O} 1$ & $125.4(2)$ \\
\hline $\mathrm{H} 8 \mathrm{~A}-\mathrm{C} 8-\mathrm{H} 8 \mathrm{~B}$ & 109.5 & $\mathrm{O} 2-\mathrm{C} 15-\mathrm{C} 9$ & $117.8(2)$ \\
\hline $\mathrm{C} 7-\mathrm{C} 8-\mathrm{H} 8 \mathrm{C}$ & 109.5 & $\mathrm{O} 1-\mathrm{C} 15-\mathrm{C} 9$ & $116.76(19)$ \\
\hline $\mathrm{H} 8 \mathrm{~A}-\mathrm{C} 8-\mathrm{H} 8 \mathrm{C}$ & 109.5 & & \\
\hline $\mathrm{C} 6-\mathrm{C} 1-\mathrm{C} 2-\mathrm{C} 3$ & $-0.4(3)$ & $\mathrm{C} 15-\mathrm{C} 9-\mathrm{C} 10-\mathrm{C} 11$ & $-176.7(2)$ \\
\hline $\mathrm{C} 7-\mathrm{C} 1-\mathrm{C} 2-\mathrm{C} 3$ & $-178.0(2)$ & $\mathrm{C} 9-\mathrm{C} 10-\mathrm{C} 11-\mathrm{C} 12$ & $0.6(3)$ \\
\hline $\mathrm{C} 1-\mathrm{C} 2-\mathrm{C} 3-\mathrm{C} 4$ & $1.1(4)$ & $\mathrm{C} 10-\mathrm{C} 11-\mathrm{C} 12-\mathrm{C} 13$ & $-1.2(3)$ \\
\hline $\mathrm{C} 2-\mathrm{C} 3-\mathrm{C} 4-\mathrm{C} 5$ & $-0.7(4)$ & $\mathrm{C} 10-\mathrm{C} 11-\mathrm{C} 12-\mathrm{I} 1$ & $177.56(16)$ \\
\hline $\mathrm{C} 3-\mathrm{C} 4-\mathrm{C} 5-\mathrm{C} 6$ & $-0.3(4)$ & $\mathrm{C} 11-\mathrm{C} 12-\mathrm{C} 13-\mathrm{C} 14$ & $0.6(3)$ \\
\hline
\end{tabular}




$\begin{array}{llll}\mathrm{C} 4-\mathrm{C} 5-\mathrm{C} 6-\mathrm{C} 1 & 1.1(4) & \mathrm{I} 1-\mathrm{C} 12-\mathrm{C} 13-\mathrm{C} 14 & -178.18(17) \\ \mathrm{C} 2-\mathrm{C} 1-\mathrm{C} 6-\mathrm{C} 5 & -0.7(3) & \mathrm{C} 12-\mathrm{C} 13-\mathrm{C} 14-\mathrm{C} 9 & 0.7(3) \\ \mathrm{C} 7-\mathrm{C} 1-\mathrm{C} 6-\mathrm{C} 5 & 177.0(2) & \mathrm{C} 10-\mathrm{C} 9-\mathrm{C} 14-\mathrm{C} 13 & -1.3(3) \\ \mathrm{C} 6-\mathrm{C} 1-\mathrm{C} 7-\mathrm{N} 1 & 142.4(2) & \mathrm{C} 15-\mathrm{C} 9-\mathrm{C} 14-\mathrm{C} 13 & 176.2(2) \\ \mathrm{C} 2-\mathrm{C} 1-\mathrm{C} 7-\mathrm{N} 1 & -40.0(3) & \mathrm{C} 10-\mathrm{C} 9-\mathrm{C} 15-\mathrm{O} 2 & -172.3(2) \\ \mathrm{C} 6-\mathrm{C} 1-\mathrm{C} 7-\mathrm{C} 8 & -94.4(3) & \mathrm{C} 14-\mathrm{C} 9-\mathrm{C} 15-\mathrm{O} 2 & 10.3(3) \\ \mathrm{C} 2-\mathrm{C} 1-\mathrm{C} 7-\mathrm{C} 8 & 83.2(3) & \mathrm{C} 10-\mathrm{C} 9-\mathrm{C} 15-\mathrm{O} 1 & 9.7(3) \\ \mathrm{C} 14-\mathrm{C} 9-\mathrm{C} 10-\mathrm{C} 11 & 0.7(3) & \mathrm{C} 14-\mathrm{C} 9-\mathrm{C} 15-\mathrm{O} 1 & -167.7(2)\end{array}$

Hydrogen-bond geometry $\left(\AA,{ }^{\circ}\right)$

\begin{tabular}{lllll}
\hline$D-\mathrm{H} \cdots A$ & $D-\mathrm{H}$ & $\mathrm{H} \cdots A$ & $D \cdots A$ & $D-\mathrm{H}^{\cdots} A$ \\
\hline $\mathrm{N} 1-\mathrm{H} 1 A \cdots \mathrm{O} 1$ & $0.88(3)$ & $1.92(3)$ & $2.796(3)$ & $175(2)$ \\
$\mathrm{N} 1-\mathrm{H} 1 B \cdots \mathrm{O} 2^{\mathrm{i}}$ & $0.84(3)$ & $1.88(3)$ & $2.715(3)$ & $174(3)$ \\
$\mathrm{N} 1-\mathrm{H} 1 C \cdots \mathrm{O} 1^{\text {ii }}$ & $0.92(3)$ & $1.83(3)$ & $2.735(2)$ & $169(3)$ \\
\hline
\end{tabular}

Symmetry codes: (i) $x, y+1, z$; (ii) $-x+3 / 2, y+1 / 2,-z+1 / 2$.

(S)-1-Cyclohexylethan-1-aminium 2-chloro-4-nitrobenzoate (V)

Crystal data

$\mathrm{C}_{8} \mathrm{H}_{18} \mathrm{~N}^{+} \cdot \mathrm{C}_{7} \mathrm{H}_{3} \mathrm{ClNO}_{4}^{-}$

$M_{r}=328.79$

Monoclinic, $C 2$

Hall symbol: $\mathrm{C} 2 \mathrm{y}$

$a=16.2280(15) \AA$

$b=6.4392(5) \AA$

$c=15.5937(15) \AA$

$\beta=104.289(4)^{\circ}$

$V=1579.1(2) \AA^{3}$

$Z=4$

\section{Data collection}

Bruker D8 Venture Photon CCD area detector diffractometer

Graphite monochromator

$\omega$ scans

Absorption correction: integration

(XPREP; Bruker, 2016)

$T_{\min }=0.910, T_{\max }=0.988$

15055 measured reflections

\section{Refinement}

Refinement on $F^{2}$

Least-squares matrix: full

$R\left[F^{2}>2 \sigma\left(F^{2}\right)\right]=0.032$

$w R\left(F^{2}\right)=0.071$

$S=1.04$

3837 reflections

211 parameters

1 restraint

0 constraints

Hydrogen site location: mixed
$F(000)=696$

$D_{\mathrm{x}}=1.383 \mathrm{Mg} \mathrm{m}^{-3}$

Mo $K \alpha$ radiation, $\lambda=0.71073 \AA$

Cell parameters from 8605 reflections

$\theta=3.2-31.0^{\circ}$

$\mu=0.26 \mathrm{~mm}^{-1}$

$T=173 \mathrm{~K}$

Plate, colourless

$0.51 \times 0.39 \times 0.06 \mathrm{~mm}$

3837 independent reflections

3587 reflections with $I>2 \sigma(I)$

$R_{\text {int }}=0.045$

$\theta_{\max }=28.0^{\circ}, \theta_{\min }=3.2^{\circ}$

$h=-21 \rightarrow 21$

$k=-8 \rightarrow 8$

$l=-20 \rightarrow 20$

$\mathrm{H}$ atoms treated by a mixture of independent and constrained refinement

$w=1 /\left[\sigma^{2}\left(F_{\mathrm{o}}^{2}\right)+(0.0364 P)^{2}+0.5174 P\right]$

where $P=\left(F_{\mathrm{o}}^{2}+2 F_{\mathrm{c}}{ }^{2}\right) / 3$

$(\Delta / \sigma)_{\max }=0.004$

$\Delta \rho_{\max }=0.20 \mathrm{e} \AA^{-3}$

$\Delta \rho_{\min }=-0.18$ e $\AA^{-3}$

Absolute structure: Flack $x$ determined using 1512 quotients $\left[\left(I^{+}\right)-\left(I^{-}\right)\right] /\left[\left(I^{+}\right)+\left(I^{\prime}\right)\right]$ (Parsons et al., 2013).

Absolute structure parameter: -0.031 (19) 


\section{Special details}

Experimental. Numerical integration absorption corrections based on indexed crystal faces were applied using the XPREP routine (Bruker, 2016)

Geometry. All esds (except the esd in the dihedral angle between two 1.s. planes) are estimated using the full covariance matrix. The cell esds are taken into account individually in the estimation of esds in distances, angles and torsion angles; correlations between esds in cell parameters are only used when they are defined by crystal symmetry. An approximate (isotropic) treatment of cell esds is used for estimating esds involving l.s. planes.

Fractional atomic coordinates and isotropic or equivalent isotropic displacement parameters $\left(\AA^{2}\right)$

\begin{tabular}{|c|c|c|c|c|}
\hline & $x$ & $y$ & $z$ & $U_{\text {iso }} * / U_{\text {eq }}$ \\
\hline $\mathrm{C} 1$ & $0.92062(12)$ & $-0.0254(3)$ & $0.72741(12)$ & $0.0222(4)$ \\
\hline H1 & 0.983347 & -0.000692 & 0.746046 & $0.027 *$ \\
\hline $\mathrm{C} 2$ & $0.87885(13)$ & $0.1383(4)$ & $0.77418(11)$ & $0.0276(4)$ \\
\hline $\mathrm{H} 2 \mathrm{~A}$ & 0.816318 & 0.121419 & 0.755933 & $0.033^{*}$ \\
\hline $\mathrm{H} 2 \mathrm{~B}$ & 0.892781 & 0.278499 & 0.755797 & $0.033 *$ \\
\hline $\mathrm{C} 3$ & $0.90886(14)$ & $0.1200(4)$ & $0.87506(12)$ & $0.0298(4)$ \\
\hline $\mathrm{H} 3 \mathrm{~A}$ & 0.97047 & 0.150661 & 0.894021 & $0.036^{*}$ \\
\hline H3B & 0.878531 & 0.223348 & 0.902958 & $0.036^{*}$ \\
\hline $\mathrm{C} 4$ & $0.89238(14)$ & -0.0959 (4) & $0.90568(13)$ & $0.0306(5)$ \\
\hline $\mathrm{H} 4 \mathrm{~A}$ & 0.830328 & -0.121076 & 0.892148 & $0.037^{*}$ \\
\hline $\mathrm{H} 4 \mathrm{~B}$ & 0.915094 & -0.106021 & 0.97058 & $0.037^{*}$ \\
\hline $\mathrm{C} 5$ & $0.93408(15)$ & $-0.2602(4)$ & $0.86040(14)$ & $0.0324(5)$ \\
\hline H5A & 0.919488 & -0.39978 & 0.878797 & $0.039 *$ \\
\hline H5B & 0.996635 & -0.244287 & 0.879238 & $0.039 *$ \\
\hline C6 & 0.90493 (14) & -0.2421 & $0.75965(13)$ & $0.0278(4)$ \\
\hline H6A & 0.935834 & -0.345333 & 0.73236 & $0.033^{*}$ \\
\hline H6B & 0.843477 & -0.274321 & 0.740347 & $0.033 *$ \\
\hline $\mathrm{C} 7$ & $0.89437(13)$ & -0.0078 & $0.62578(12)$ & $0.0245(4)$ \\
\hline $\mathrm{H} 7$ & 0.913554 & -0.13714 & 0.600976 & $0.029 *$ \\
\hline $\mathrm{C} 8$ & $0.93451(14)$ & $0.1751(4)$ & $0.59025(14)$ & $0.0345(5)$ \\
\hline H8A & 0.996473 & 0.168179 & 0.612246 & $0.052 *$ \\
\hline H8B & 0.91942 & 0.170527 & 0.525424 & $0.052^{*}$ \\
\hline $\mathrm{H} 8 \mathrm{C}$ & 0.913611 & 0.304811 & 0.61009 & $0.052 *$ \\
\hline C9 & $0.67604(12)$ & $0.3841(3)$ & $0.70870(12)$ & $0.0195(4)$ \\
\hline $\mathrm{C} 10$ & $0.63111(11)$ & $0.1984(3)$ & $0.68641(12)$ & $0.0199(4)$ \\
\hline C11 & $0.61854(12)$ & 0.0605 & $0.74977(13)$ & $0.0224(4)$ \\
\hline H11 & 0.589347 & -0.067073 & 0.733844 & $0.027^{*}$ \\
\hline $\mathrm{C} 12$ & $0.65033(12)$ & 0.1160 & $0.83759(12)$ & $0.0236(4)$ \\
\hline $\mathrm{C} 13$ & $0.69029(14)$ & 0.3020 & $0.86335(13)$ & $0.0265(4)$ \\
\hline H13 & 0.708769 & 0.338067 & 0.924157 & $0.032 *$ \\
\hline $\mathrm{C} 14$ & $0.70281(13)$ & $0.4352(3)$ & $0.79816(12)$ & $0.0244(4)$ \\
\hline H14 & 0.730308 & 0.564495 & 0.814713 & $0.029 *$ \\
\hline $\mathrm{C} 15$ & $0.70359(12)$ & $0.5207(3)$ & $0.64162(12)$ & $0.0206(4)$ \\
\hline N1 & $0.79918(11)$ & 0.0076 & $0.59071(10)$ & 0.0213 \\
\hline N2 & $0.64113(13)$ & $-0.0332(3)$ & $0.90616(12)$ & $0.0327(4)$ \\
\hline $\mathrm{O} 1$ & $0.74062(9)$ & $0.4280(2)$ & $0.59039(9)$ & $0.0255(3)$ \\
\hline $\mathrm{O} 2$ & $0.69186(10)$ & $0.7100(2)$ & $0.64544(10)$ & 0.0288 \\
\hline
\end{tabular}




\begin{tabular}{lllll} 
O3 & $0.61108(15)$ & $-0.2043(3)$ & $0.88266(13)$ & $0.0522(5)$ \\
O4 & $0.66539(14)$ & $0.0207(3)$ & $0.98356(11)$ & $0.0488(5)$ \\
C11 & $0.58672(3)$ & $0.13764(8)$ & $0.57637(3)$ & $0.03040(13)$ \\
H1A & $0.7792(15)$ & $0.139(5)$ & $0.5991(15)$ & $0.030(6)^{*}$ \\
H1B & $0.7824(16)$ & $-0.011(4)$ & $0.5295(17)$ & $0.032(6)^{*}$ \\
H1C & $0.7717(16)$ & $-0.091(4)$ & $0.6117(17)$ & $0.028(6)^{*}$ \\
\hline
\end{tabular}

Atomic displacement parameters $\left(\AA^{2}\right)$

\begin{tabular}{lllllll}
\hline & $U^{11}$ & $U^{22}$ & $U^{33}$ & $U^{12}$ & $U^{13}$ & $U^{23}$ \\
\hline C1 & $0.0220(9)$ & $0.0239(9)$ & $0.0206(9)$ & $0.0011(8)$ & $0.0051(7)$ & $-0.0010(7)$ \\
C2 & $0.0404(10)$ & $0.0216(8)$ & $0.0208(8)$ & $0.0048(10)$ & $0.0076(7)$ & $-0.0005(9)$ \\
C3 & $0.0385(10)$ & $0.0301(11)$ & $0.0204(8)$ & $0.0028(10)$ & $0.0063(8)$ & $-0.0052(9)$ \\
C4 & $0.0323(11)$ & $0.0393(13)$ & $0.0199(9)$ & $0.0020(10)$ & $0.0060(8)$ & $0.0038(9)$ \\
C5 & $0.0374(12)$ & $0.0304(11)$ & $0.0271(10)$ & $0.0064(10)$ & $0.0033(9)$ & $0.0070(8)$ \\
C6 & $0.0328(11)$ & $0.0233(10)$ & $0.0259(10)$ & $0.0036(9)$ & $0.0047(8)$ & $-0.0001(8)$ \\
C7 & $0.0259(10)$ & $0.0284(10)$ & $0.0201(9)$ & $0.0008(8)$ & $0.0078(7)$ & $-0.0020(8)$ \\
C8 & $0.0345(11)$ & $0.0439(15)$ & $0.0270(10)$ & $-0.0082(10)$ & $0.0114(8)$ & $0.0029(9)$ \\
C9 & $0.0203(8)$ & $0.0188(9)$ & $0.0197(9)$ & $0.0028(7)$ & $0.0054(7)$ & $0.0007(7)$ \\
C10 & $0.0181(8)$ & $0.0231(9)$ & $0.0170(8)$ & $0.0015(7)$ & $0.0013(7)$ & $-0.0010(6)$ \\
C11 & $0.0206(9)$ & $0.0211(9)$ & $0.0260(9)$ & $-0.0004(7)$ & $0.0068(7)$ & $0.0002(7)$ \\
C12 & $0.0239(8)$ & $0.0269(10)$ & $0.0227(8)$ & $0.0034(9)$ & $0.0106(7)$ & $0.0062(8)$ \\
C13 & $0.0304(10)$ & $0.0330(11)$ & $0.0168(9)$ & $0.0004(9)$ & $0.0075(8)$ & $-0.0016(8)$ \\
C14 & $0.0300(10)$ & $0.0237(9)$ & $0.0204(9)$ & $-0.0036(8)$ & $0.0075(8)$ & $-0.0047(7)$ \\
C15 & $0.0210(9)$ & $0.0216(10)$ & $0.0178(8)$ & $-0.0005(8)$ & $0.0021(7)$ & $0.0015(7)$ \\
N1 & $0.0278(9)$ & $0.0195(8)$ & $0.0175(8)$ & $-0.0011(7)$ & $0.0073(6)$ & $0.0000(6)$ \\
N2 & $0.0385(10)$ & $0.0340(10)$ & $0.0309(10)$ & $0.0101(8)$ & $0.0187(8)$ & $0.0118(8)$ \\
O1 & $0.0337(8)$ & $0.0258(7)$ & $0.0195(6)$ & $0.0030(6)$ & $0.0112(6)$ & $0.0026(6)$ \\
O2 & $0.0376(8)$ & $0.0197(7)$ & $0.0322(8)$ & $0.0007(6)$ & $0.0143(7)$ & $0.0031(6)$ \\
O3 & $0.0801(15)$ & $0.0340(10)$ & $0.0509(11)$ & $-0.0100(10)$ & $0.0321(10)$ & $0.0099(8)$ \\
O4 & $0.0711(13)$ & $0.0519(11)$ & $0.0261(8)$ & $0.0079(10)$ & $0.0171(8)$ & $0.0137(8)$ \\
C11 & $0.0323(2)$ & $0.0334(2)$ & $0.0200(2)$ & $-0.0058(2)$ & $-0.00398(16)$ & $-0.0016(2)$ \\
& & & & & & \\
& & & & & & \\
& & & & & & \\
& & & & &
\end{tabular}

Geometric parameters $\left(\AA,{ }^{\circ}\right)$

\begin{tabular}{llll}
\hline $\mathrm{C} 1-\mathrm{C} 6$ & $1.525(3)$ & $\mathrm{C} 8-\mathrm{H} 8 \mathrm{~B}$ & 0.98 \\
$\mathrm{C} 1-\mathrm{C} 2$ & $1.532(3)$ & $\mathrm{C} 8-\mathrm{H} 8 \mathrm{C}$ & 0.98 \\
$\mathrm{C} 1-\mathrm{C} 7$ & $1.540(3)$ & $\mathrm{C} 9-\mathrm{C} 14$ & $1.394(3)$ \\
$\mathrm{C} 1-\mathrm{H} 1$ & 1 & $\mathrm{C} 9-\mathrm{C} 10$ & $1.399(3)$ \\
$\mathrm{C} 2-\mathrm{C} 3$ & $1.532(2)$ & $\mathrm{C} 9-\mathrm{C} 15$ & $1.516(3)$ \\
$\mathrm{C} 2-\mathrm{H} 2 \mathrm{~A}$ & 0.99 & $\mathrm{C} 10-\mathrm{C} 11$ & $1.381(3)$ \\
$\mathrm{C} 2-\mathrm{H} 2 \mathrm{~B}$ & 0.99 & $\mathrm{C} 10-\mathrm{C} 11$ & $1.7340(18)$ \\
$\mathrm{C} 3-\mathrm{C} 4$ & $1.515(3)$ & $\mathrm{C} 11-\mathrm{C} 12$ & $1.386(3)$ \\
$\mathrm{C} 3-\mathrm{H} 3 \mathrm{~A}$ & 0.99 & $\mathrm{C} 11-\mathrm{H} 11$ & 0.95 \\
$\mathrm{C} 3-\mathrm{H} 3 \mathrm{~B}$ & 0.99 & $\mathrm{C} 12-\mathrm{C} 13$ & $1.374(3)$ \\
$\mathrm{C} 4-\mathrm{C} 5$ & $1.520(3)$ & $\mathrm{C} 12-\mathrm{N} 2$ & $1.472(3)$ \\
$\mathrm{C} 4-\mathrm{H} 4 \mathrm{~A}$ & 0.99 & $\mathrm{C} 13-\mathrm{C} 14$ & $1.383(3)$ \\
$\mathrm{C} 4-\mathrm{H} 4 \mathrm{~B}$ & 0.99 & $\mathrm{C} 13-\mathrm{H} 13$ & 0.95
\end{tabular}




\begin{tabular}{|c|c|c|c|}
\hline $\mathrm{C} 5-\mathrm{C} 6$ & $1.529(3)$ & $\mathrm{C} 14-\mathrm{H} 14$ & 0.95 \\
\hline C5-H5A & 0.99 & $\mathrm{C} 15-\mathrm{O} 2$ & $1.238(2)$ \\
\hline C5-H5B & 0.99 & $\mathrm{C} 15-\mathrm{O} 1$ & $1.262(2)$ \\
\hline C6-H6A & 0.99 & $\mathrm{~N} 1-\mathrm{H} 1 \mathrm{~A}$ & $0.93(3)$ \\
\hline $\mathrm{C} 6-\mathrm{H} 6 \mathrm{~B}$ & 0.99 & $\mathrm{~N} 1-\mathrm{H} 1 \mathrm{~B}$ & $0.93(3)$ \\
\hline $\mathrm{C} 7-\mathrm{N} 1$ & $1.509(3)$ & $\mathrm{N} 1-\mathrm{H} 1 \mathrm{C}$ & $0.88(3)$ \\
\hline $\mathrm{C} 7-\mathrm{C} 8$ & $1.515(3)$ & $\mathrm{N} 2-\mathrm{O} 4$ & $1.224(3)$ \\
\hline $\mathrm{C} 7-\mathrm{H} 7$ & 1 & $\mathrm{~N} 2-\mathrm{O} 3$ & $1.224(3)$ \\
\hline $\mathrm{C} 8-\mathrm{H} 8 \mathrm{~A}$ & 0.98 & & \\
\hline $\mathrm{C} 6-\mathrm{C} 1-\mathrm{C} 2$ & $110.06(16)$ & $\mathrm{N} 1-\mathrm{C} 7-\mathrm{H} 7$ & 107.6 \\
\hline $\mathrm{C} 6-\mathrm{C} 1-\mathrm{C} 7$ & $112.35(16)$ & $\mathrm{C} 8-\mathrm{C} 7-\mathrm{H} 7$ & 107.6 \\
\hline $\mathrm{C} 2-\mathrm{C} 1-\mathrm{C} 7$ & $113.37(16)$ & $\mathrm{C} 1-\mathrm{C} 7-\mathrm{H} 7$ & 107.6 \\
\hline $\mathrm{C} 6-\mathrm{C} 1-\mathrm{H} 1$ & 106.9 & $\mathrm{C} 7-\mathrm{C} 8-\mathrm{H} 8 \mathrm{~A}$ & 109.5 \\
\hline $\mathrm{C} 2-\mathrm{C} 1-\mathrm{H} 1$ & 106.9 & $\mathrm{C} 7-\mathrm{C} 8-\mathrm{H} 8 \mathrm{~B}$ & 109.5 \\
\hline $\mathrm{C} 7-\mathrm{C} 1-\mathrm{H} 1$ & 106.9 & $\mathrm{H} 8 \mathrm{~A}-\mathrm{C} 8-\mathrm{H} 8 \mathrm{~B}$ & 109.5 \\
\hline $\mathrm{C} 1-\mathrm{C} 2-\mathrm{C} 3$ & $111.71(17)$ & $\mathrm{C} 7-\mathrm{C} 8-\mathrm{H} 8 \mathrm{C}$ & 109.5 \\
\hline $\mathrm{C} 1-\mathrm{C} 2-\mathrm{H} 2 \mathrm{~A}$ & 109.3 & $\mathrm{H} 8 \mathrm{~A}-\mathrm{C} 8-\mathrm{H} 8 \mathrm{C}$ & 109.5 \\
\hline $\mathrm{C} 3-\mathrm{C} 2-\mathrm{H} 2 \mathrm{~A}$ & 109.3 & $\mathrm{H} 8 \mathrm{~B}-\mathrm{C} 8-\mathrm{H} 8 \mathrm{C}$ & 109.5 \\
\hline $\mathrm{C} 1-\mathrm{C} 2-\mathrm{H} 2 \mathrm{~B}$ & 109.3 & $\mathrm{C} 14-\mathrm{C} 9-\mathrm{C} 10$ & $117.70(17)$ \\
\hline $\mathrm{C} 3-\mathrm{C} 2-\mathrm{H} 2 \mathrm{~B}$ & 109.3 & $\mathrm{C} 14-\mathrm{C} 9-\mathrm{C} 15$ & $118.77(17)$ \\
\hline $\mathrm{H} 2 \mathrm{~A}-\mathrm{C} 2-\mathrm{H} 2 \mathrm{~B}$ & 107.9 & $\mathrm{C} 10-\mathrm{C} 9-\mathrm{C} 15$ & $123.29(17)$ \\
\hline $\mathrm{C} 4-\mathrm{C} 3-\mathrm{C} 2$ & $110.99(18)$ & $\mathrm{C} 11-\mathrm{C} 10-\mathrm{C} 9$ & $122.15(17)$ \\
\hline $\mathrm{C} 4-\mathrm{C} 3-\mathrm{H} 3 \mathrm{~A}$ & 109.4 & $\mathrm{C} 11-\mathrm{C} 10-\mathrm{C} 11$ & $117.66(15)$ \\
\hline $\mathrm{C} 2-\mathrm{C} 3-\mathrm{H} 3 \mathrm{~A}$ & 109.4 & $\mathrm{C} 9-\mathrm{C} 10-\mathrm{Cl} 1$ & $120.17(14)$ \\
\hline $\mathrm{C} 4-\mathrm{C} 3-\mathrm{H} 3 \mathrm{~B}$ & 109.4 & $\mathrm{C} 10-\mathrm{C} 11-\mathrm{C} 12$ & $117.15(18)$ \\
\hline $\mathrm{C} 2-\mathrm{C} 3-\mathrm{H} 3 \mathrm{~B}$ & 109.4 & $\mathrm{C} 10-\mathrm{C} 11-\mathrm{H} 11$ & 121.4 \\
\hline $\mathrm{H} 3 \mathrm{~A}-\mathrm{C} 3-\mathrm{H} 3 \mathrm{~B}$ & 108 & $\mathrm{C} 12-\mathrm{C} 11-\mathrm{H} 11$ & 121.4 \\
\hline $\mathrm{C} 3-\mathrm{C} 4-\mathrm{C} 5$ & $111.02(17)$ & $\mathrm{C} 13-\mathrm{C} 12-\mathrm{C} 11$ & $123.21(18)$ \\
\hline $\mathrm{C} 3-\mathrm{C} 4-\mathrm{H} 4 \mathrm{~A}$ & 109.4 & $\mathrm{C} 13-\mathrm{C} 12-\mathrm{N} 2$ & $118.79(17)$ \\
\hline $\mathrm{C} 5-\mathrm{C} 4-\mathrm{H} 4 \mathrm{~A}$ & 109.4 & $\mathrm{C} 11-\mathrm{C} 12-\mathrm{N} 2$ & $118.00(19)$ \\
\hline $\mathrm{C} 3-\mathrm{C} 4-\mathrm{H} 4 \mathrm{~B}$ & 109.4 & $\mathrm{C} 12-\mathrm{C} 13-\mathrm{C} 14$ & $118.03(17)$ \\
\hline $\mathrm{C} 5-\mathrm{C} 4-\mathrm{H} 4 \mathrm{~B}$ & 109.4 & $\mathrm{C} 12-\mathrm{C} 13-\mathrm{H} 13$ & 121 \\
\hline $\mathrm{H} 4 \mathrm{~A}-\mathrm{C} 4-\mathrm{H} 4 \mathrm{~B}$ & 108 & $\mathrm{C} 14-\mathrm{C} 13-\mathrm{H} 13$ & 121 \\
\hline $\mathrm{C} 4-\mathrm{C} 5-\mathrm{C} 6$ & $111.33(17)$ & $\mathrm{C} 13-\mathrm{C} 14-\mathrm{C} 9$ & $121.56(19)$ \\
\hline $\mathrm{C} 4-\mathrm{C} 5-\mathrm{H} 5 \mathrm{~A}$ & 109.4 & $\mathrm{C} 13-\mathrm{C} 14-\mathrm{H} 14$ & 119.2 \\
\hline $\mathrm{C} 6-\mathrm{C} 5-\mathrm{H} 5 \mathrm{~A}$ & 109.4 & $\mathrm{C} 9-\mathrm{C} 14-\mathrm{H} 14$ & 119.2 \\
\hline $\mathrm{C} 4-\mathrm{C} 5-\mathrm{H} 5 \mathrm{~B}$ & 109.4 & $\mathrm{O} 2-\mathrm{C} 15-\mathrm{O} 1$ & $126.8(2)$ \\
\hline $\mathrm{C} 6-\mathrm{C} 5-\mathrm{H} 5 \mathrm{~B}$ & 109.4 & $\mathrm{O} 2-\mathrm{C} 15-\mathrm{C} 9$ & $117.60(18)$ \\
\hline $\mathrm{H} 5 \mathrm{~A}-\mathrm{C} 5-\mathrm{H} 5 \mathrm{~B}$ & 108 & $\mathrm{O} 1-\mathrm{C} 15-\mathrm{C} 9$ & $115.48(17)$ \\
\hline $\mathrm{C} 1-\mathrm{C} 6-\mathrm{C} 5$ & $111.95(17)$ & $\mathrm{C} 7-\mathrm{N} 1-\mathrm{H} 1 \mathrm{~A}$ & $111.8(15)$ \\
\hline $\mathrm{C} 1-\mathrm{C} 6-\mathrm{H} 6 \mathrm{~A}$ & 109.2 & $\mathrm{C} 7-\mathrm{N} 1-\mathrm{H} 1 \mathrm{~B}$ & $112.1(15)$ \\
\hline $\mathrm{C} 5-\mathrm{C} 6-\mathrm{H} 6 \mathrm{~A}$ & 109.2 & $\mathrm{H} 1 \mathrm{~A}-\mathrm{N} 1-\mathrm{H} 1 \mathrm{~B}$ & $104(2)$ \\
\hline $\mathrm{C} 1-\mathrm{C} 6-\mathrm{H} 6 \mathrm{~B}$ & 109.2 & $\mathrm{C} 7-\mathrm{N} 1-\mathrm{H} 1 \mathrm{C}$ & $112.6(17)$ \\
\hline $\mathrm{C} 5-\mathrm{C} 6-\mathrm{H} 6 \mathrm{~B}$ & 109.2 & $\mathrm{H} 1 \mathrm{~A}-\mathrm{N} 1-\mathrm{H} 1 \mathrm{C}$ & $112(2)$ \\
\hline $\mathrm{H} 6 \mathrm{~A}-\mathrm{C} 6-\mathrm{H} 6 \mathrm{~B}$ & 107.9 & $\mathrm{H} 1 \mathrm{~B}-\mathrm{N} 1-\mathrm{H} 1 \mathrm{C}$ & $104(2)$ \\
\hline $\mathrm{N} 1-\mathrm{C} 7-\mathrm{C} 8$ & 108.09 (17) & $\mathrm{O} 4-\mathrm{N} 2-\mathrm{O} 3$ & $123.90(19)$ \\
\hline $\mathrm{N} 1-\mathrm{C} 7-\mathrm{C} 1$ & $112.08(15)$ & $\mathrm{O} 4-\mathrm{N} 2-\mathrm{C} 12$ & $117.8(2)$ \\
\hline
\end{tabular}




$\begin{array}{llll}\mathrm{C} 8-\mathrm{C} 7-\mathrm{C} 1 & 113.51(17) & \mathrm{O} 3-\mathrm{N} 2-\mathrm{C} 12 & 118.32(19) \\ \mathrm{C} 6-\mathrm{C} 1-\mathrm{C} 2-\mathrm{C} 3 & 55.1(2) & \mathrm{C} 11-\mathrm{C} 10-\mathrm{C} 11-\mathrm{C} 12 & -176.58(14) \\ \mathrm{C} 7-\mathrm{C} 1-\mathrm{C} 2-\mathrm{C} 3 & -178.10(17) & \mathrm{C} 10-\mathrm{C} 11-\mathrm{C} 12-\mathrm{C} 13 & 2.3(3) \\ \mathrm{C} 1-\mathrm{C} 2-\mathrm{C} 3-\mathrm{C} 4 & -56.3(2) & \mathrm{C} 10-\mathrm{C} 11-\mathrm{C} 12-\mathrm{N} 2 & -177.19(17) \\ \mathrm{C} 2-\mathrm{C} 3-\mathrm{C} 4-\mathrm{C} 5 & 56.1(2) & \mathrm{C} 11-\mathrm{C} 12-\mathrm{C} 13-\mathrm{C} 14 & -3.2(3) \\ \mathrm{C} 3-\mathrm{C} 4-\mathrm{C} 5-\mathrm{C} 6 & -55.7(2) & \mathrm{N} 2-\mathrm{C} 12-\mathrm{C} 13-\mathrm{C} 14 & 176.29(18) \\ \mathrm{C} 2-\mathrm{C} 1-\mathrm{C} 6-\mathrm{C} 5 & -54.7(2) & \mathrm{C} 12-\mathrm{C} 13-\mathrm{C} 14-\mathrm{C} 9 & 0.0(3) \\ \mathrm{C} 7-\mathrm{C} 1-\mathrm{C} 6-\mathrm{C} 5 & 177.98(16) & \mathrm{C} 10-\mathrm{C} 9-\mathrm{C} 14-\mathrm{C} 13 & 3.9(3) \\ \mathrm{C} 4-\mathrm{C} 5-\mathrm{C} 6-\mathrm{C} 1 & 55.5(2) & \mathrm{C} 15-\mathrm{C} 9-\mathrm{C} 14-\mathrm{C} 13 & -170.65(18) \\ \mathrm{C} 6-\mathrm{C} 1-\mathrm{C} 7-\mathrm{N} 1 & 76.3(2) & \mathrm{C} 14-\mathrm{C} 9-\mathrm{C} 15-\mathrm{O} 2 & -50.9(3) \\ \mathrm{C} 2-\mathrm{C} 1-\mathrm{C} 7-\mathrm{N} 1 & -49.2(2) & \mathrm{C} 10-\mathrm{C} 9-\mathrm{C} 15-\mathrm{O} 2 & 134.9(2) \\ \mathrm{C} 6-\mathrm{C} 1-\mathrm{C} 7-\mathrm{C} 8 & -160.87(18) & \mathrm{C} 14-\mathrm{C} 9-\mathrm{C} 15-\mathrm{O} 1 & 125.41(19) \\ \mathrm{C} 2-\mathrm{C} 1-\mathrm{C} 7-\mathrm{C} 8 & 73.6(2) & \mathrm{C} 10-\mathrm{C} 9-\mathrm{C} 15-\mathrm{O} 1 & -48.8(3) \\ \mathrm{C} 14-\mathrm{C} 9-\mathrm{C} 10-\mathrm{C} 11 & -4.8(3) & \mathrm{C} 13-\mathrm{C} 12-\mathrm{N} 2-\mathrm{O} 4 & 4.1(3) \\ \mathrm{C} 15-\mathrm{C} 9-\mathrm{C} 10-\mathrm{C} 11 & 169.42(18) & \mathrm{C} 11-\mathrm{C} 12-\mathrm{N} 2-\mathrm{O} 4 & -176.37(19) \\ \mathrm{C} 14-\mathrm{C} 9-\mathrm{C} 10-\mathrm{C} 11 & 173.55(15) & \mathrm{C} 13-\mathrm{C} 12-\mathrm{N} 2-\mathrm{O} 3 & -174.7(2) \\ \mathrm{C} 15-\mathrm{C} 9-\mathrm{C} 10-\mathrm{C} 11 & -12.2(3) & \mathrm{C} 11-\mathrm{C} 12-\mathrm{N} 2-\mathrm{O} 3 & 4.8(3) \\ \mathrm{C} 9-\mathrm{C} 10-\mathrm{C} 11-\mathrm{C} 12 & 1.9(3) & & \end{array}$

Hydrogen-bond geometry $\left(\AA,{ }^{\circ}\right)$

\begin{tabular}{lllll}
\hline$D-\mathrm{H} \cdots A$ & $D-\mathrm{H}$ & $\mathrm{H} \cdots A$ & $D \cdots A$ & $D-\mathrm{H} \cdots A$ \\
\hline $\mathrm{N} 1-\mathrm{H} 1 A^{\cdots} \cdots \mathrm{O} 1$ & $0.93(3)$ & $1.96(3)$ & $2.869(2)$ & $167(2)$ \\
$\mathrm{N} 1-\mathrm{H} 1 B \cdots \mathrm{O} 1^{\mathrm{i}}$ & $0.93(3)$ & $1.86(3)$ & $2.785(2)$ & $173(2)$ \\
$\mathrm{N} 1-\mathrm{H} 1 C \cdots \mathrm{O} 2^{\mathrm{ii}}$ & $0.88(3)$ & $1.99(3)$ & $2.858(2)$ & $170(2)$ \\
\hline
\end{tabular}

Symmetry codes: (i) $-x+3 / 2, y-1 / 2,-z+1$; (ii) $x, y-1, z$.

2-(Cyclohex-1-en-1-yl)ethan-1-aminium 4-bromobenzoate (VI)

Crystal data

$\mathrm{C}_{8} \mathrm{H}_{15} \mathrm{~N}^{+} \cdot \mathrm{C}_{7} \mathrm{H}_{4} \mathrm{BrO}_{2}^{-}$

$M_{r}=325.22$

Monoclinic, $P 2{ }_{1} / n$

Hall symbol: -P 2yn

$a=6.4391(3) \AA$

$b=17.0023(8) \AA$

$c=14.1588(6) \AA$

$\beta=102.241(2)^{\circ}$

$V=1514.86(12) \AA^{3}$

$Z=4$

\section{Data collection}

Bruker D8 Venture Photon CCD area detector diffractometer

Graphite monochromator

$\omega$ scans

Absorption correction: integration

(XPREP; Bruker, 2016)

$T_{\min }=0.275, T_{\max }=0.776$

30862 measured reflections
$F(000)=668$

$D_{\mathrm{x}}=1.426 \mathrm{Mg} \mathrm{m}^{-3}$

Mo $K \alpha$ radiation, $\lambda=0.71073 \AA$

Cell parameters from 9914 reflections

$\theta=3.2-28.3^{\circ}$

$\mu=2.71 \mathrm{~mm}^{-1}$

$T=173 \mathrm{~K}$

Rods, colourless

$0.68 \times 0.18 \times 0.1 \mathrm{~mm}$

3658 independent reflections

3302 reflections with $I>2 \sigma(I)$

$R_{\text {int }}=0.071$

$\theta_{\max }=28.0^{\circ}, \theta_{\min }=2.8^{\circ}$

$h=-8 \rightarrow 8$

$k=-22 \rightarrow 22$

$l=-18 \rightarrow 18$ 


\section{Refinement}

Refinement on $F^{2}$

Least-squares matrix: full

$R\left[F^{2}>2 \sigma\left(F^{2}\right)\right]=0.037$

$w R\left(F^{2}\right)=0.098$

$S=1.05$

3658 reflections

194 parameters

0 restraints

0 constraints
Hydrogen site location: mixed

$\mathrm{H}$ atoms treated by a mixture of independent and constrained refinement

$w=1 /\left[\sigma^{2}\left(F_{\mathrm{o}}{ }^{2}\right)+(0.0477 P)^{2}+1.5945 P\right]$

where $P=\left(F_{\mathrm{o}}^{2}+2 F_{\mathrm{c}}{ }^{2}\right) / 3$

$(\Delta / \sigma)_{\max }=0.001$

$\Delta \rho_{\max }=1.01 \mathrm{e} \AA^{-3}$

$\Delta \rho_{\text {min }}=-1.02$ e $\AA^{-3}$

\section{Special details}

Experimental. Numerical integration absorption corrections based on indexed crystal faces were applied using the XPREP routine (Bruker, 2016)

Geometry. All esds (except the esd in the dihedral angle between two 1.s. planes) are estimated using the full covariance matrix. The cell esds are taken into account individually in the estimation of esds in distances, angles and torsion angles; correlations between esds in cell parameters are only used when they are defined by crystal symmetry. An approximate (isotropic) treatment of cell esds is used for estimating esds involving l.s. planes.

Fractional atomic coordinates and isotropic or equivalent isotropic displacement parameters $\left(\AA^{2}\right)$

\begin{tabular}{|c|c|c|c|c|c|}
\hline & $x$ & $y$ & $z$ & $U_{\text {iso }} * / U_{\text {eq }}$ & Occ. $(<1)$ \\
\hline $\mathrm{C} 1$ & 0.9952 (4) & $0.55176(13)$ & $0.35862(16)$ & $0.0297(5)$ & \\
\hline $\mathrm{C} 2$ & $1.2265(4)$ & $0.56748(17)$ & 0.38957 (19) & $0.0394(6)$ & \\
\hline $\mathrm{H} 2 \mathrm{~A}$ & 1.272267 & 0.599559 & 0.339287 & $0.047 *$ & \\
\hline $\mathrm{H} 2 \mathrm{~B}$ & 1.303568 & 0.516784 & 0.393878 & $0.047^{*}$ & \\
\hline $\mathrm{C} 3$ & $1.2903(4)$ & $0.6097(2)$ & $0.4859(2)$ & $0.0482(7)$ & \\
\hline $\mathrm{H} 3 \mathrm{~A}$ & 1.29862 & 0.571138 & 0.538886 & $0.058 *$ & $0.77(2)$ \\
\hline H3B & 1.433308 & 0.632993 & 0.491224 & $0.058 *$ & $0.77(2)$ \\
\hline $\mathrm{H} 3 \mathrm{C}$ & 1.367424 & 0.571718 & 0.533684 & $0.058 *$ & $0.23(2)$ \\
\hline H3D & 1.39227 & 0.651718 & 0.47853 & $0.058 *$ & $0.23(2)$ \\
\hline $\mathrm{C} 4 \mathrm{~A}$ & $1.1367(7)$ & $0.6733(4)$ & $0.4972(5)$ & $0.0337(12)$ & $0.77(2)$ \\
\hline H4A & 1.137787 & 0.714401 & 0.447738 & $0.04 *$ & $0.77(2)$ \\
\hline H4B & 1.182544 & 0.697969 & 0.561611 & $0.04 *$ & $0.77(2)$ \\
\hline C4B & $1.126(3)$ & 0.6445 (19) & $0.5257(16)$ & $0.046(5)$ & $0.23(2)$ \\
\hline $\mathrm{H} 4 \mathrm{C}$ & 1.143892 & 0.622775 & 0.591802 & $0.055^{*}$ & $0.23(2)$ \\
\hline H4D & 1.161962 & 0.701067 & 0.533582 & $0.055^{*}$ & $0.23(2)$ \\
\hline $\mathrm{C} 5$ & $0.9110(4)$ & $0.64165(17)$ & $0.4868(2)$ & $0.0432(6)$ & \\
\hline H5A & 0.818606 & 0.655783 & 0.528143 & $0.052 *$ & $0.77(2)$ \\
\hline H5B & 0.809547 & 0.672265 & 0.510406 & $0.052 *$ & $0.23(2)$ \\
\hline C6 & $0.8522(4)$ & $0.58524(15)$ & $0.40311(17)$ & $0.0337(5)$ & \\
\hline H6 & 0.706363 & 0.572658 & 0.380575 & $0.04 *$ & \\
\hline $\mathrm{C} 7$ & 0.9225 & $0.49832(14)$ & $0.27304(16)$ & $0.0342(5)$ & \\
\hline H7A & 0.77838 & 0.478269 & 0.273552 & $0.041^{*}$ & \\
\hline H7B & 1.019715 & 0.452701 & 0.277803 & $0.041^{*}$ & \\
\hline $\mathrm{C} 8$ & 0.9188 & $0.54181(13)$ & $0.17902(15)$ & 0.0278 & \\
\hline H8A & 0.833004 & 0.590296 & 0.177594 & $0.033^{*}$ & \\
\hline H8B & 1.065383 & 0.557518 & 0.176224 & $0.033^{*}$ & \\
\hline C9 & $0.2632(3)$ & $0.32298(12)$ & $0.19291(14)$ & $0.0213(4)$ & \\
\hline $\mathrm{C} 10$ & 0.4191 & $0.29872(13)$ & $0.27194(15)$ & $0.0253(4)$ & \\
\hline
\end{tabular}




$\begin{array}{lllll}\text { H10 } & 0.559631 & 0.318645 & 0.279912 & 0.03^{*} \\ \text { C11 } & 0.3711(3) & 0.24585(13) & 0.33893(15) & 0.0271(4) \\ \text { H11 } & 0.477044 & 0.229697 & 0.392839 & 0.033^{*} \\ \text { C12 } & 0.1660(3) & 0.21718(12) & 0.32558(15) & 0.0240(4) \\ \text { C13 } & 0.0081(3) & 0.23954(13) & 0.24739(16) & 0.0278(4) \\ \text { H13 } & -0.131731 & 0.218908 & 0.239255 & 0.033^{*} \\ \text { C14 } & 0.0588(3) & 0.29276(13) & 0.18123(15) & 0.0257(4) \\ \text { H14 } & -0.047572 & 0.308672 & 0.127369 & 0.031^{*} \\ \text { C15 } & 0.3204(3) & 0.38114(12) & 0.12140(15) & 0.0237(4) \\ \text { N1 } & 0.8286(3) & 0.49309(12) & 0.09315(13) & 0.0242(4) \\ \text { O1 } & 0.5104(3) & 0.39903(11) & 0.13033(13) & 0.0366(4) \\ \text { O2 } & 0.1681(3) & 0.40722(10) & 0.05740(12) & 0.0327(4) \\ \text { Br1 } & 0.09846(4) & 0.14745(2) & 0.41940(2) & 0.03754(10) \\ \text { H1A } & 0.716(5) & 0.4690(18) & 0.100(2) & 0.039(8)^{*} \\ \text { H1B } & 0.928(5) & 0.456(2) & 0.082(2) & 0.042(8)^{*} \\ \text { H1C } & 0.809(5) & 0.5244(19) & 0.045(2) & 0.042(8)^{*}\end{array}$

Atomic displacement parameters $\left(\AA^{2}\right)$

\begin{tabular}{lllllll}
\hline & $U^{11}$ & $U^{22}$ & $U^{33}$ & $U^{12}$ & $U^{13}$ & $U^{23}$ \\
\hline C1 & $0.0350(11)$ & $0.0303(11)$ & $0.0227(10)$ & $-0.0081(9)$ & $0.0036(8)$ & $0.0016(8)$ \\
C2 & $0.0284(11)$ & $0.0489(15)$ & $0.0409(13)$ & $-0.0036(10)$ & $0.0075(10)$ & $-0.0104(11)$ \\
C3 & $0.0314(12)$ & $0.0606(18)$ & $0.0491(16)$ & $-0.0057(12)$ & $0.0004(11)$ & $-0.0145(14)$ \\
C4A & $0.0330(17)$ & $0.037(2)$ & $0.030(2)$ & $-0.0094(16)$ & $0.0043(15)$ & $-0.0051(17)$ \\
C4B & $0.052(8)$ & $0.056(13)$ & $0.028(8)$ & $-0.010(8)$ & $0.006(6)$ & $-0.010(7)$ \\
C5 & $0.0326(12)$ & $0.0538(16)$ & $0.0428(14)$ & $-0.0036(11)$ & $0.0072(11)$ & $-0.0164(12)$ \\
C6 & $0.0263(10)$ & $0.0398(12)$ & $0.0339(12)$ & $-0.0060(9)$ & $0.0042(9)$ & $-0.0052(10)$ \\
C7 & $0.0464(13)$ & $0.0322(11)$ & $0.0229(10)$ & $-0.0104(10)$ & $0.0049(9)$ & $0.0003(9)$ \\
C8 & $0.0304(11)$ & $0.0277(10)$ & $0.0242(10)$ & $-0.0053(8)$ & $0.0037(8)$ & $0.0021(8)$ \\
C9 & $0.0226(9)$ & $0.0218(9)$ & $0.0205(9)$ & $0.0003(7)$ & $0.0069(7)$ & $-0.0016(7)$ \\
C10 & $0.0199(9)$ & $0.0289(10)$ & $0.0262(10)$ & $-0.0027(8)$ & $0.0029(7)$ & $-0.0005(8)$ \\
C11 & $0.0250(10)$ & $0.0307(11)$ & $0.0232(9)$ & $0.0004(8)$ & $-0.0002(8)$ & $0.0029(8)$ \\
C12 & $0.0267(10)$ & $0.0213(9)$ & $0.0249(10)$ & $-0.0002(7)$ & $0.0074(8)$ & $0.0032(7)$ \\
C13 & $0.0203(9)$ & $0.0309(11)$ & $0.0316(11)$ & $-0.0030(8)$ & $0.0041(8)$ & $0.0059(9)$ \\
C14 & $0.0218(9)$ & $0.0288(10)$ & $0.0248(10)$ & $-0.0007(8)$ & $0.0007(7)$ & $0.0041(8)$ \\
C15 & $0.0279(10)$ & $0.0233(9)$ & $0.0223(9)$ & $-0.0006(8)$ & $0.0105(8)$ & $-0.0014(7)$ \\
N1 & $0.0229(8)$ & $0.0297(9)$ & $0.0204(8)$ & $-0.0029(7)$ & $0.0055(7)$ & $0.0039(7)$ \\
O1 & $0.0305(8)$ & $0.0442(10)$ & $0.0365(9)$ & $-0.0120(7)$ & $0.0101(7)$ & $0.0054(7)$ \\
O2 & $0.0322(8)$ & $0.0390(9)$ & $0.0291(8)$ & $0.0059(7)$ & $0.0112(6)$ & $0.0137(7)$ \\
Br1 & $0.03653(15)$ & $0.03802(16)$ & $0.03819(15)$ & $-0.00282(9)$ & $0.00818(10)$ & $0.01726(10)$ \\
& & & & & & \\
\hline
\end{tabular}

Geometric parameters $\left(\AA,{ }^{\circ}\right)$

\begin{tabular}{llll}
\hline $\mathrm{C} 1-\mathrm{C} 6$ & $1.347(3)$ & $\mathrm{C} 7-\mathrm{H} 7 \mathrm{~A}$ & 0.99 \\
$\mathrm{C} 1-\mathrm{C} 2$ & $1.485(3)$ & $\mathrm{C} 7-\mathrm{H} 7 \mathrm{~B}$ & 0.99 \\
$\mathrm{C} 1-\mathrm{C} 7$ & $1.508(3)$ & $\mathrm{C} 8-\mathrm{N} 1$ & $1.484(3)$ \\
$\mathrm{C} 2-\mathrm{C} 3$ & $1.519(4)$ & $\mathrm{C} 8-\mathrm{H} 8 \mathrm{~A}$ & 0.99 \\
$\mathrm{C} 2-\mathrm{H} 2 \mathrm{~A}$ & 0.99 & $\mathrm{C} 8-\mathrm{H} 8 \mathrm{~B}$ & 0.99
\end{tabular}




\begin{tabular}{|c|c|c|c|}
\hline $\mathrm{C} 2-\mathrm{H} 2 \mathrm{~B}$ & 0.99 & $\mathrm{C} 9-\mathrm{C} 14$ & $1.390(3)$ \\
\hline $\mathrm{C} 3-\mathrm{C} 4 \mathrm{~B}$ & $1.429(18)$ & $\mathrm{C} 9-\mathrm{C} 10$ & $1.398(3)$ \\
\hline $\mathrm{C} 3-\mathrm{C} 4 \mathrm{~A}$ & $1.497(6)$ & $\mathrm{C} 9-\mathrm{C} 15$ & $1.515(3)$ \\
\hline $\mathrm{C} 3-\mathrm{H} 3 \mathrm{~A}$ & 0.99 & $\mathrm{C} 10-\mathrm{C} 11$ & $1.388(3)$ \\
\hline $\mathrm{C} 3-\mathrm{H} 3 \mathrm{~B}$ & 0.99 & $\mathrm{C} 10-\mathrm{H} 10$ & 0.95 \\
\hline $\mathrm{C} 3-\mathrm{H} 3 \mathrm{C}$ & 0.99 & $\mathrm{C} 11-\mathrm{C} 12$ & $1.383(3)$ \\
\hline $\mathrm{C} 3-\mathrm{H} 3 \mathrm{D}$ & 0.99 & $\mathrm{C} 11-\mathrm{H} 11$ & 0.95 \\
\hline $\mathrm{C} 4 \mathrm{~A}-\mathrm{C} 5$ & $1.527(5)$ & $\mathrm{C} 12-\mathrm{C} 13$ & $1.388(3)$ \\
\hline $\mathrm{C} 4 \mathrm{~A}-\mathrm{H} 4 \mathrm{~A}$ & 0.99 & $\mathrm{C} 12-\mathrm{Br} 1$ & $1.898(2)$ \\
\hline $\mathrm{C} 4 \mathrm{~A}-\mathrm{H} 4 \mathrm{~B}$ & 0.99 & $\mathrm{C} 13-\mathrm{C} 14$ & $1.390(3)$ \\
\hline $\mathrm{C} 4 \mathrm{~B}-\mathrm{C} 5$ & $1.377(18)$ & $\mathrm{C} 13-\mathrm{H} 13$ & 0.95 \\
\hline $\mathrm{C} 4 \mathrm{~B}-\mathrm{H} 4 \mathrm{C}$ & 0.99 & $\mathrm{C} 14-\mathrm{H} 14$ & 0.95 \\
\hline $\mathrm{C} 4 \mathrm{~B}-\mathrm{H} 4 \mathrm{D}$ & 0.99 & $\mathrm{C} 15-\mathrm{O} 1$ & $1.241(3)$ \\
\hline $\mathrm{C} 5-\mathrm{C} 6$ & $1.509(3)$ & $\mathrm{C} 15-\mathrm{O} 2$ & $1.266(3)$ \\
\hline $\mathrm{C} 5-\mathrm{H} 5 \mathrm{~A}$ & 0.95 & $\mathrm{~N} 1-\mathrm{H} 1 \mathrm{~A}$ & $0.86(3)$ \\
\hline $\mathrm{C} 5-\mathrm{H} 5 \mathrm{~B}$ & 0.95 & $\mathrm{~N} 1-\mathrm{H} 1 \mathrm{~B}$ & $0.94(3)$ \\
\hline $\mathrm{C} 6-\mathrm{H} 6$ & 0.95 & $\mathrm{~N} 1-\mathrm{H} 1 \mathrm{C}$ & $0.86(3)$ \\
\hline $\mathrm{C} 7-\mathrm{C} 8$ & $1.519(3)$ & & \\
\hline $\mathrm{C} 6-\mathrm{C} 1-\mathrm{C} 2$ & $121.9(2)$ & $\mathrm{C} 5-\mathrm{C} 6-\mathrm{H} 6$ & 118.2 \\
\hline $\mathrm{C} 6-\mathrm{C} 1-\mathrm{C} 7$ & $120.3(2)$ & $\mathrm{C} 1-\mathrm{C} 7-\mathrm{C} 8$ & $110.83(19)$ \\
\hline $\mathrm{C} 2-\mathrm{C} 1-\mathrm{C} 7$ & $117.8(2)$ & $\mathrm{C} 1-\mathrm{C} 7-\mathrm{H} 7 \mathrm{~A}$ & 109.5 \\
\hline $\mathrm{C} 1-\mathrm{C} 2-\mathrm{C} 3$ & $114.4(2)$ & $\mathrm{C} 8-\mathrm{C} 7-\mathrm{H} 7 \mathrm{~A}$ & 109.5 \\
\hline $\mathrm{C} 1-\mathrm{C} 2-\mathrm{H} 2 \mathrm{~A}$ & 108.7 & $\mathrm{C} 1-\mathrm{C} 7-\mathrm{H} 7 \mathrm{~B}$ & 109.5 \\
\hline $\mathrm{C} 3-\mathrm{C} 2-\mathrm{H} 2 \mathrm{~A}$ & 108.7 & $\mathrm{C} 8-\mathrm{C} 7-\mathrm{H} 7 \mathrm{~B}$ & 109.5 \\
\hline $\mathrm{C} 1-\mathrm{C} 2-\mathrm{H} 2 \mathrm{~B}$ & 108.7 & $\mathrm{H} 7 \mathrm{~A}-\mathrm{C} 7-\mathrm{H} 7 \mathrm{~B}$ & 108.1 \\
\hline $\mathrm{C} 3-\mathrm{C} 2-\mathrm{H} 2 \mathrm{~B}$ & 108.7 & $\mathrm{~N} 1-\mathrm{C} 8-\mathrm{C} 7$ & $112.16(18)$ \\
\hline $\mathrm{H} 2 \mathrm{~A}-\mathrm{C} 2-\mathrm{H} 2 \mathrm{~B}$ & 107.6 & $\mathrm{~N} 1-\mathrm{C} 8-\mathrm{H} 8 \mathrm{~A}$ & 109.2 \\
\hline $\mathrm{C} 4 \mathrm{~B}-\mathrm{C} 3-\mathrm{C} 2$ & $117.9(7)$ & $\mathrm{C} 7-\mathrm{C} 8-\mathrm{H} 8 \mathrm{~A}$ & 109.2 \\
\hline $\mathrm{C} 4 \mathrm{~A}-\mathrm{C} 3-\mathrm{C} 2$ & $112.3(3)$ & $\mathrm{N} 1-\mathrm{C} 8-\mathrm{H} 8 \mathrm{~B}$ & 109.2 \\
\hline $\mathrm{C} 4 \mathrm{~A}-\mathrm{C} 3-\mathrm{H} 3 \mathrm{~A}$ & 109.2 & $\mathrm{C} 7-\mathrm{C} 8-\mathrm{H} 8 \mathrm{~B}$ & 109.2 \\
\hline $\mathrm{C} 2-\mathrm{C} 3-\mathrm{H} 3 \mathrm{~A}$ & 109.2 & $\mathrm{H} 8 \mathrm{~A}-\mathrm{C} 8-\mathrm{H} 8 \mathrm{~B}$ & 107.9 \\
\hline $\mathrm{C} 4 \mathrm{~A}-\mathrm{C} 3-\mathrm{H} 3 \mathrm{~B}$ & 109.2 & $\mathrm{C} 14-\mathrm{C} 9-\mathrm{C} 10$ & $119.17(19)$ \\
\hline $\mathrm{C} 2-\mathrm{C} 3-\mathrm{H} 3 \mathrm{~B}$ & 109.2 & $\mathrm{C} 14-\mathrm{C} 9-\mathrm{C} 15$ & $121.33(18)$ \\
\hline $\mathrm{H} 3 \mathrm{~A}-\mathrm{C} 3-\mathrm{H} 3 \mathrm{~B}$ & 107.9 & $\mathrm{C} 10-\mathrm{C} 9-\mathrm{C} 15$ & $119.49(18)$ \\
\hline $\mathrm{C} 4 \mathrm{~B}-\mathrm{C} 3-\mathrm{H} 3 \mathrm{C}$ & 107.8 & $\mathrm{C} 11-\mathrm{C} 10-\mathrm{C} 9$ & $120.78(19)$ \\
\hline $\mathrm{C} 2-\mathrm{C} 3-\mathrm{H} 3 \mathrm{C}$ & 107.8 & $\mathrm{C} 11-\mathrm{C} 10-\mathrm{H} 10$ & 119.6 \\
\hline $\mathrm{C} 4 \mathrm{~B}-\mathrm{C} 3-\mathrm{H} 3 \mathrm{D}$ & 107.8 & $\mathrm{C} 9-\mathrm{C} 10-\mathrm{H} 10$ & 119.6 \\
\hline $\mathrm{C} 2-\mathrm{C} 3-\mathrm{H} 3 \mathrm{D}$ & 107.8 & $\mathrm{C} 12-\mathrm{C} 11-\mathrm{C} 10$ & $118.66(19)$ \\
\hline $\mathrm{H} 3 \mathrm{C}-\mathrm{C} 3-\mathrm{H} 3 \mathrm{D}$ & 107.2 & $\mathrm{C} 12-\mathrm{C} 11-\mathrm{H} 11$ & 120.7 \\
\hline $\mathrm{C} 3-\mathrm{C} 4 \mathrm{~A}-\mathrm{C} 5$ & $111.8(4)$ & $\mathrm{C} 10-\mathrm{C} 11-\mathrm{H} 11$ & 120.7 \\
\hline $\mathrm{C} 3-\mathrm{C} 4 \mathrm{~A}-\mathrm{H} 4 \mathrm{~A}$ & 109.3 & $\mathrm{C} 11-\mathrm{C} 12-\mathrm{C} 13$ & $121.97(19)$ \\
\hline $\mathrm{C} 5-\mathrm{C} 4 \mathrm{~A}-\mathrm{H} 4 \mathrm{~A}$ & 109.3 & $\mathrm{C} 11-\mathrm{C} 12-\mathrm{Br} 1$ & $118.58(15)$ \\
\hline $\mathrm{C} 3-\mathrm{C} 4 \mathrm{~A}-\mathrm{H} 4 \mathrm{~B}$ & 109.3 & $\mathrm{C} 13-\mathrm{C} 12-\mathrm{Br} 1$ & $119.43(16)$ \\
\hline $\mathrm{C} 5-\mathrm{C} 4 \mathrm{~A}-\mathrm{H} 4 \mathrm{~B}$ & 109.3 & $\mathrm{C} 12-\mathrm{C} 13-\mathrm{C} 14$ & $118.59(19)$ \\
\hline $\mathrm{H} 4 \mathrm{~A}-\mathrm{C} 4 \mathrm{~A}-\mathrm{H} 4 \mathrm{~B}$ & 107.9 & $\mathrm{C} 12-\mathrm{C} 13-\mathrm{H} 13$ & 120.7 \\
\hline $\mathrm{C} 5-\mathrm{C} 4 \mathrm{~B}-\mathrm{C} 3$ & $126.4(11)$ & $\mathrm{C} 14-\mathrm{C} 13-\mathrm{H} 13$ & 120.7 \\
\hline $\mathrm{C} 5-\mathrm{C} 4 \mathrm{~B}-\mathrm{H} 4 \mathrm{C}$ & 105.7 & $\mathrm{C} 9-\mathrm{C} 14-\mathrm{C} 13$ & $120.83(19)$ \\
\hline
\end{tabular}




\begin{tabular}{|c|c|c|c|}
\hline $\mathrm{C} 3-\mathrm{C} 4 \mathrm{~B}-\mathrm{H} 4 \mathrm{C}$ & 105.7 & $\mathrm{C} 9-\mathrm{C} 14-\mathrm{H} 14$ & 119.6 \\
\hline $\mathrm{C} 5-\mathrm{C} 4 \mathrm{~B}-\mathrm{H} 4 \mathrm{D}$ & 105.7 & $\mathrm{C} 13-\mathrm{C} 14-\mathrm{H} 14$ & 119.6 \\
\hline $\mathrm{C} 3-\mathrm{C} 4 \mathrm{~B}-\mathrm{H} 4 \mathrm{D}$ & 105.7 & $\mathrm{O} 1-\mathrm{C} 15-\mathrm{O} 2$ & $125.8(2)$ \\
\hline $\mathrm{H} 4 \mathrm{C}-\mathrm{C} 4 \mathrm{~B}-\mathrm{H} 4 \mathrm{D}$ & 106.2 & $\mathrm{O} 1-\mathrm{C} 15-\mathrm{C} 9$ & $117.77(19)$ \\
\hline $\mathrm{C} 4 \mathrm{~B}-\mathrm{C} 5-\mathrm{C} 6$ & $113.6(7)$ & $\mathrm{O} 2-\mathrm{C} 15-\mathrm{C} 9$ & $116.46(18)$ \\
\hline $\mathrm{C} 6-\mathrm{C} 5-\mathrm{C} 4 \mathrm{~A}$ & $112.0(3)$ & $\mathrm{C} 8-\mathrm{N} 1-\mathrm{H} 1 \mathrm{~A}$ & $111(2)$ \\
\hline $\mathrm{C} 6-\mathrm{C} 5-\mathrm{H} 5 \mathrm{~A}$ & 124 & $\mathrm{C} 8-\mathrm{N} 1-\mathrm{H} 1 \mathrm{~B}$ & $110.6(18)$ \\
\hline $\mathrm{C} 4 \mathrm{~A}-\mathrm{C} 5-\mathrm{H} 5 \mathrm{~A}$ & 124 & $\mathrm{H} 1 \mathrm{~A}-\mathrm{N} 1-\mathrm{H} 1 \mathrm{~B}$ & $109(3)$ \\
\hline $\mathrm{C} 4 \mathrm{~B}-\mathrm{C} 5-\mathrm{H} 5 \mathrm{~B}$ & 123.2 & $\mathrm{C} 8-\mathrm{N} 1-\mathrm{H} 1 \mathrm{C}$ & $106(2)$ \\
\hline $\mathrm{C} 6-\mathrm{C} 5-\mathrm{H} 5 \mathrm{~B}$ & 123.2 & $\mathrm{H} 1 \mathrm{~A}-\mathrm{N} 1-\mathrm{H} 1 \mathrm{C}$ & $114(3)$ \\
\hline $\mathrm{C} 1-\mathrm{C} 6-\mathrm{C} 5$ & $123.6(2)$ & $\mathrm{H} 1 \mathrm{~B}-\mathrm{N} 1-\mathrm{H} 1 \mathrm{C}$ & $106(3)$ \\
\hline $\mathrm{C} 1-\mathrm{C} 6-\mathrm{H} 6$ & 118.2 & & \\
\hline $\mathrm{C} 6-\mathrm{C} 1-\mathrm{C} 2-\mathrm{C} 3$ & $11.9(4)$ & $\mathrm{C} 14-\mathrm{C} 9-\mathrm{C} 10-\mathrm{C} 11$ & $-0.8(3)$ \\
\hline $\mathrm{C} 7-\mathrm{C} 1-\mathrm{C} 2-\mathrm{C} 3$ & $-169.8(2)$ & $\mathrm{C} 15-\mathrm{C} 9-\mathrm{C} 10-\mathrm{C} 11$ & $179.63(19)$ \\
\hline $\mathrm{C} 1-\mathrm{C} 2-\mathrm{C} 3-\mathrm{C} 4 \mathrm{~B}$ & $-13.2(17)$ & $\mathrm{C} 9-\mathrm{C} 10-\mathrm{C} 11-\mathrm{C} 12$ & $0.4(3)$ \\
\hline $\mathrm{C} 1-\mathrm{C} 2-\mathrm{C} 3-\mathrm{C} 4 \mathrm{~A}$ & $-40.6(5)$ & $\mathrm{C} 10-\mathrm{C} 11-\mathrm{C} 12-\mathrm{C} 13$ & $0.2(3)$ \\
\hline $\mathrm{C} 2-\mathrm{C} 3-\mathrm{C} 4 \mathrm{~A}-\mathrm{C} 5$ & $57.2(6)$ & $\mathrm{C} 10-\mathrm{C} 11-\mathrm{C} 12-\mathrm{Br} 1$ & $-178.05(16)$ \\
\hline $\mathrm{C} 2-\mathrm{C} 3-\mathrm{C} 4 \mathrm{~B}-\mathrm{C} 5$ & $2(4)$ & $\mathrm{C} 11-\mathrm{C} 12-\mathrm{C} 13-\mathrm{C} 14$ & $-0.4(3)$ \\
\hline $\mathrm{C} 3-\mathrm{C} 4 \mathrm{~B}-\mathrm{C} 5-\mathrm{C} 6$ & $10(4)$ & $\mathrm{Br} 1-\mathrm{C} 12-\mathrm{C} 13-\mathrm{C} 14$ & $177.80(16)$ \\
\hline $\mathrm{C} 3-\mathrm{C} 4 \mathrm{~A}-\mathrm{C} 5-\mathrm{C} 6$ & $-43.8(6)$ & $\mathrm{C} 10-\mathrm{C} 9-\mathrm{C} 14-\mathrm{C} 13$ & $0.5(3)$ \\
\hline $\mathrm{C} 2-\mathrm{C} 1-\mathrm{C} 6-\mathrm{C} 5$ & $0.6(4)$ & $\mathrm{C} 15-\mathrm{C} 9-\mathrm{C} 14-\mathrm{C} 13$ & $-179.9(2)$ \\
\hline $\mathrm{C} 7-\mathrm{C} 1-\mathrm{C} 6-\mathrm{C} 5$ & $-177.7(2)$ & $\mathrm{C} 12-\mathrm{C} 13-\mathrm{C} 14-\mathrm{C} 9$ & $0.1(3)$ \\
\hline $\mathrm{C} 4 \mathrm{~B}-\mathrm{C} 5-\mathrm{C} 6-\mathrm{C} 1$ & $-11.7(17)$ & $\mathrm{C} 14-\mathrm{C} 9-\mathrm{C} 15-\mathrm{O} 1$ & $-172.3(2)$ \\
\hline $\mathrm{C} 4 \mathrm{~A}-\mathrm{C} 5-\mathrm{C} 6-\mathrm{C} 1$ & $15.5(5)$ & $\mathrm{C} 10-\mathrm{C} 9-\mathrm{C} 15-\mathrm{O} 1$ & $7.3(3)$ \\
\hline $\mathrm{C} 6-\mathrm{C} 1-\mathrm{C} 7-\mathrm{C} 8$ & $100.0(3)$ & $\mathrm{C} 14-\mathrm{C} 9-\mathrm{C} 15-\mathrm{O} 2$ & $7.7(3)$ \\
\hline $\mathrm{C} 2-\mathrm{C} 1-\mathrm{C} 7-\mathrm{C} 8$ & $-78.4(3)$ & $\mathrm{C} 10-\mathrm{C} 9-\mathrm{C} 15-\mathrm{O} 2$ & $-172.70(19)$ \\
\hline $\mathrm{C} 1-\mathrm{C} 7-\mathrm{C} 8-\mathrm{N} 1$ & $-174.6(2)$ & & \\
\hline
\end{tabular}

Hydrogen-bond geometry $\left(A,{ }^{\circ}\right)$

\begin{tabular}{lllll}
\hline$D-\mathrm{H} \cdots A$ & $D-\mathrm{H}$ & $\mathrm{H} \cdots A$ & $D \cdots A$ & $D-\mathrm{H} \cdots A$ \\
\hline $\mathrm{N} 1-\mathrm{H} 1 A \cdots \mathrm{O} 1$ & $0.86(3)$ & $1.89(3)$ & $2.737(2)$ & $167(3)$ \\
$\mathrm{N} 1-\mathrm{H} 1 B \cdots \mathrm{O} 2^{\mathrm{i}}$ & $0.94(3)$ & $1.85(3)$ & $2.763(3)$ & $164(3)$ \\
$\mathrm{N} 1-\mathrm{H} 1 C \cdots \mathrm{O} 2^{\mathrm{ii}}$ & $0.86(3)$ & $1.89(3)$ & $2.727(2)$ & $167(3)$ \\
\hline
\end{tabular}

Symmetry codes: (i) $x+1, y, z$; (ii) $-x+1,-y+1,-z$.

(S)-1-Cyclohexylethan-1-aminium 4-bromobenzoate' (VII)

Crystal data

$\mathrm{C}_{8} \mathrm{H}_{18} \mathrm{~N}^{+} \cdot \mathrm{C}_{7} \mathrm{H}_{4} \mathrm{BrO}_{2}^{-}$

$M_{r}=328.24$

Orthorhombic, $P 2_{1} 2_{1} 2_{1}$

Hall symbol: P $2 \mathrm{ac} 2 \mathrm{ab}$

$a=6.2790(3) \AA$

$b=15.6610(9) \AA$

$c=15.8800(8) \AA$

$V=1561.57(14) \AA^{3}$

$Z=4$
$F(000)=680$

$D_{\mathrm{x}}=1.396 \mathrm{Mg} \mathrm{m}^{-3}$

Mo $K \alpha$ radiation, $\lambda=0.71073 \AA$

Cell parameters from 9958 reflections

$\theta=3.5-28.1^{\circ}$

$\mu=2.63 \mathrm{~mm}^{-1}$

$T=173 \mathrm{~K}$

Needle, colourless

$0.69 \times 0.13 \times 0.10 \mathrm{~mm}$ 


\section{Data collection}

Bruker D8 Venture Photon CCD area detector diffractometer

Graphite monochromator

$\omega$ scans

Absorption correction: integration

(XPREP; Bruker, 2016)

$T_{\min }=0.452, T_{\max }=0.846$

21006 measured reflections

\section{Refinement}

Refinement on $F^{2}$

Least-squares matrix: full

$R\left[F^{2}>2 \sigma\left(F^{2}\right)\right]=0.078$

$w R\left(F^{2}\right)=0.211$

$S=1.08$

2913 reflections

174 parameters

0 restraints

0 constraints

Hydrogen site location: inferred from neighbouring sites
2913 independent reflections

2687 reflections with $I>2 \sigma(I)$

$R_{\text {int }}=0.068$

$\theta_{\max }=25.5^{\circ}, \theta_{\min }=2.9^{\circ}$

$h=-7 \rightarrow 7$

$k=-18 \rightarrow 18$

$l=-19 \rightarrow 19$
H-atom parameters constrained

$w=1 /\left[\sigma^{2}\left(F_{\mathrm{o}}^{2}\right)+(0.1114 P)^{2}+6.6115 P\right]$

where $P=\left(F_{\mathrm{o}}{ }^{2}+2 F_{\mathrm{c}}{ }^{2}\right) / 3$

$(\Delta / \sigma)_{\max }<0.001$

$\Delta \rho_{\max }=1.46 \mathrm{e} \AA^{-3}$

$\Delta \rho_{\min }=-0.48$ e $\AA^{-3}$

Absolute structure: Flack $x$ determined using 1026 quotients $\left[\left(I^{+}\right)-(I)\right] /\left[\left(I^{+}\right)+\left(I^{-}\right)\right]$(Parsons et al., 2013)

Absolute structure parameter: 0.068 (9)

\section{Special details}

Experimental. Numerical integration absorption corrections based on indexed crystal faces were applied using the XPREP routine (Bruker, 2016)

Geometry. All esds (except the esd in the dihedral angle between two 1.s. planes) are estimated using the full covariance matrix. The cell esds are taken into account individually in the estimation of esds in distances, angles and torsion angles; correlations between esds in cell parameters are only used when they are defined by crystal symmetry. An approximate (isotropic) treatment of cell esds is used for estimating esds involving l.s. planes.

Fractional atomic coordinates and isotropic or equivalent isotropic displacement parameters $\left(\AA^{2}\right)$

\begin{tabular}{lllll}
\hline & $x$ & $y$ & $z$ & $U_{\text {iso }} / U_{\text {eq }}$ \\
\hline C1 & $0.773(2)$ & $0.0775(8)$ & $0.3199(7)$ & $0.053(3)$ \\
H1 & 0.740942 & 0.015006 & 0.317739 & $0.064^{*}$ \\
C2 & $0.612(2)$ & $0.1220(11)$ & $0.2688(8)$ & $0.068(4)$ \\
H2A & 0.638924 & 0.184264 & 0.269256 & $0.082^{*}$ \\
H2B & 0.467953 & 0.111721 & 0.292398 & $0.082^{*}$ \\
C3 & $0.624(3)$ & $0.0862(13)$ & $0.1743(9)$ & $0.084(5)$ \\
H3A & 0.595663 & 0.024063 & 0.173633 & $0.1^{*}$ \\
H3B & 0.516609 & 0.114896 & 0.138545 & $0.1^{*}$ \\
C4 & $0.844(2)$ & $0.1039(11)$ & $0.1413(8)$ & $0.077(5)$ \\
H4A & 0.85316 & 0.08645 & 0.081516 & $0.093^{*}$ \\
H4B & 0.873874 & 0.165911 & 0.144629 & $0.093^{*}$ \\
C5 & $1.005(3)$ & $0.0565(12)$ & $0.1911(10)$ & $0.083(5)$ \\
H5A & 1.149674 & 0.066472 & 0.168707 & $0.1^{*}$ \\
H5B & 0.974778 & -0.005537 & 0.19016 & $0.1^{*}$ \\
C6 & $0.984(3)$ & $0.0910(11)$ & $0.2780(10)$ & $0.080(5)$ \\
H6A & 1.013382 & 0.153146 & 0.276219 & $0.097^{*}$ \\
H6B & 1.096015 & 0.064487 & 0.313464 & $0.097^{*}$ \\
C7 & $0.762(2)$ & $0.1051(7)$ & $0.4107(7)$ & $0.056(3)$ \\
& & & &
\end{tabular}




$\begin{array}{lllll}\text { H7 } & 0.882202 & 0.075639 & 0.44015 & 0.067^{*} \\ \text { C8 } & 0.563(3) & 0.0786(10) & 0.4553(8) & 0.086(6) \\ \text { H8A } & 0.468938 & 0.128083 & 0.461722 & 0.129^{*} \\ \text { H8B } & 0.599286 & 0.05585 & 0.510964 & 0.129^{*} \\ \text { H8C } & 0.490145 & 0.034387 & 0.422389 & 0.129^{*} \\ \text { N1 } & 0.7938(13) & 0.1998(5) & 0.4253(4) & 0.0331(16) \\ \text { H1A } & 0.691137 & 0.229515 & 0.397416 & 0.05^{*} \\ \text { H1B } & 0.924273 & 0.215652 & 0.40592 & 0.05^{*} \\ \text { H1C } & 0.784863 & 0.21113 & 0.481408 & 0.05^{*} \\ \text { C9 } & 0.4242(17) & 0.3282(6) & 0.2666(6) & 0.036(2) \\ \text { C10 } & 0.631(2) & 0.3587(7) & 0.2719(7) & 0.051(3) \\ \text { H10 } & 0.690085 & 0.370093 & 0.325852 & 0.061^{*} \\ \text { C11 } & 0.7566(19) & 0.3734(7) & 0.2006(6) & 0.047(2) \\ \text { H11 } & 0.899884 & 0.392507 & 0.204207 & 0.057^{*} \\ \text { C12 } & 0.6544(19) & 0.3577(6) & 0.1225(7) & 0.046(2) \\ \text { C13 } & 0.4508(18) & 0.3286(7) & 0.1153(7) & 0.043(2) \\ \text { H13 } & 0.394001 & 0.31832 & 0.0608 & 0.052^{*} \\ \text { C14 } & 0.321(2) & 0.3131(6) & 0.1868(6) & 0.043(2) \\ \text { H14 } & 0.177757 & 0.294286 & 0.182502 & 0.052^{*} \\ \text { C15 } & 0.2958(14) & 0.3029(6) & 0.3460(5) & 0.0276(18) \\ \text { O1 } & 0.4108(15) & 0.2878(6) & 0.4104(5) & 0.061(2) \\ \text { O2 } & 0.1043(14) & 0.3044(6) & 0.3421(5) & 0.058(2) \\ \text { Br1 } & 0.8185(2) & 0.37723(9) & 0.02444(6) & 0.0586(4) \\ & & & & \end{array}$

Atomic displacement parameters $\left(\AA^{2}\right)$

\begin{tabular}{lllllll}
\hline & $U^{11}$ & $U^{22}$ & $U^{33}$ & $U^{12}$ & $U^{13}$ & $U^{23}$ \\
\hline $\mathrm{C} 1$ & $0.059(7)$ & $0.061(6)$ & $0.040(6)$ & $-0.010(6)$ & $0.009(5)$ & $-0.008(5)$ \\
$\mathrm{C} 2$ & $0.067(8)$ & $0.080(8)$ & $0.058(7)$ & $0.024(8)$ & $0.014(6)$ & $-0.014(7)$ \\
$\mathrm{C} 3$ & $0.083(10)$ & $0.126(14)$ & $0.043(7)$ & $0.023(9)$ & $-0.018(7)$ & $-0.023(8)$ \\
$\mathrm{C} 4$ & $0.080(9)$ & $0.110(12)$ & $0.042(6)$ & $0.018(9)$ & $0.026(7)$ & $0.019(7)$ \\
$\mathrm{C} 5$ & $0.083(11)$ & $0.104(12)$ & $0.063(9)$ & $0.005(10)$ & $-0.005(8)$ & $-0.020(9)$ \\
$\mathrm{C} 6$ & $0.068(9)$ & $0.098(11)$ & $0.076(10)$ & $-0.020(8)$ & $0.018(8)$ & $-0.044(8)$ \\
$\mathrm{C} 7$ & $0.081(9)$ & $0.047(6)$ & $0.039(6)$ & $-0.012(5)$ & $-0.009(5)$ & $-0.004(5)$ \\
$\mathrm{C} 8$ & $0.137(15)$ & $0.080(9)$ & $0.042(7)$ & $-0.059(10)$ & $0.017(8)$ & $-0.014(6)$ \\
$\mathrm{N} 1$ & $0.032(4)$ & $0.050(4)$ & $0.018(3)$ & $0.002(4)$ & $-0.004(3)$ & $0.001(3)$ \\
$\mathrm{C} 9$ & $0.040(5)$ & $0.040(5)$ & $0.028(5)$ & $-0.008(4)$ & $0.003(4)$ & $0.004(4)$ \\
$\mathrm{C} 10$ & $0.074(8)$ & $0.046(6)$ & $0.033(5)$ & $0.002(5)$ & $0.004(5)$ & $0.002(4)$ \\
$\mathrm{C} 11$ & $0.070(7)$ & $0.045(5)$ & $0.027(4)$ & $-0.010(5)$ & $0.003(4)$ & $0.001(4)$ \\
$\mathrm{C} 12$ & $0.055(6)$ & $0.044(5)$ & $0.038(5)$ & $0.007(5)$ & $0.011(5)$ & $-0.001(4)$ \\
$\mathrm{C} 13$ & $0.049(6)$ & $0.048(6)$ & $0.032(5)$ & $0.003(5)$ & $-0.003(5)$ & $-0.002(4)$ \\
$\mathrm{C} 14$ & $0.066(7)$ & $0.036(4)$ & $0.026(5)$ & $0.001(5)$ & $-0.005(5)$ & $0.006(4)$ \\
$\mathrm{C} 15$ & $0.025(4)$ & $0.040(4)$ & $0.018(4)$ & $0.005(4)$ & $0.006(3)$ & $0.001(3)$ \\
O1 & $0.059(5)$ & $0.093(6)$ & $0.031(4)$ & $0.015(5)$ & $0.007(4)$ & $0.008(4)$ \\
O2 & $0.048(5)$ & $0.082(6)$ & $0.044(5)$ & $-0.001(4)$ & $0.010(4)$ & $0.013(4)$ \\
Br1 & $0.0598(7)$ & $0.0877(8)$ & $0.0283(5)$ & $0.0091(7)$ & $0.0127(5)$ & $0.0065(5)$ \\
& & & & & & \\
\hline
\end{tabular}


Geometric parameters $\left(\AA,{ }^{\circ}\right)$

\begin{tabular}{|c|c|c|c|}
\hline $\mathrm{C} 1-\mathrm{C} 2$ & $1.475(19)$ & $\mathrm{C} 8-\mathrm{H} 8 \mathrm{~A}$ & 0.98 \\
\hline $\mathrm{C} 1-\mathrm{C} 6$ & $1.498(19)$ & $\mathrm{C} 8-\mathrm{H} 8 \mathrm{~B}$ & 0.98 \\
\hline $\mathrm{C} 1-\mathrm{C} 7$ & $1.507(16)$ & $\mathrm{C} 8-\mathrm{H} 8 \mathrm{C}$ & 0.98 \\
\hline $\mathrm{C} 1-\mathrm{H} 1$ & 1 & $\mathrm{~N} 1-\mathrm{H} 1 \mathrm{~A}$ & 0.91 \\
\hline $\mathrm{C} 2-\mathrm{C} 3$ & $1.604(18)$ & $\mathrm{N} 1-\mathrm{H} 1 \mathrm{~B}$ & 0.91 \\
\hline $\mathrm{C} 2-\mathrm{H} 2 \mathrm{~A}$ & 0.99 & $\mathrm{~N} 1-\mathrm{H} 1 \mathrm{C}$ & 0.91 \\
\hline $\mathrm{C} 2-\mathrm{H} 2 \mathrm{~B}$ & 0.99 & $\mathrm{C} 9-\mathrm{C} 10$ & $1.389(17)$ \\
\hline $\mathrm{C} 3-\mathrm{C} 4$ & $1.50(2)$ & $\mathrm{C} 9-\mathrm{C} 14$ & $1.442(14)$ \\
\hline $\mathrm{C} 3-\mathrm{H} 3 \mathrm{~A}$ & 0.99 & $\mathrm{C} 9-\mathrm{C} 15$ & $1.548(12)$ \\
\hline $\mathrm{C} 3-\mathrm{H} 3 \mathrm{~B}$ & 0.99 & $\mathrm{C} 10-\mathrm{C} 11$ & $1.397(15)$ \\
\hline $\mathrm{C} 4-\mathrm{C} 5$ & $1.48(2)$ & $\mathrm{C} 10-\mathrm{H} 10$ & 0.95 \\
\hline $\mathrm{C} 4-\mathrm{H} 4 \mathrm{~A}$ & 0.99 & $\mathrm{C} 11-\mathrm{C} 12$ & $1.418(15)$ \\
\hline $\mathrm{C} 4-\mathrm{H} 4 \mathrm{~B}$ & 0.99 & $\mathrm{C} 11-\mathrm{H} 11$ & 0.95 \\
\hline $\mathrm{C} 5-\mathrm{C} 6$ & $1.49(2)$ & $\mathrm{C} 12-\mathrm{C} 13$ & $1.362(17)$ \\
\hline $\mathrm{C} 5-\mathrm{H} 5 \mathrm{~A}$ & 0.99 & $\mathrm{C} 12-\mathrm{Br} 1$ & $1.892(11)$ \\
\hline C5-H5B & 0.99 & $\mathrm{C} 13-\mathrm{C} 14$ & $1.418(15)$ \\
\hline C6-H6A & 0.99 & $\mathrm{C} 13-\mathrm{H} 13$ & 0.95 \\
\hline C6-H6B & 0.99 & C14-H14 & 0.95 \\
\hline $\mathrm{C} 7-\mathrm{C} 8$ & $1.50(2)$ & $\mathrm{C} 15-\mathrm{O} 2$ & $1.204(12)$ \\
\hline $\mathrm{C} 7-\mathrm{N} 1$ & $1.515(13)$ & $\mathrm{C} 15-\mathrm{O} 1$ & $1.273(12)$ \\
\hline $\mathrm{C} 7-\mathrm{H} 7$ & 1 & & \\
\hline $\mathrm{C} 2-\mathrm{C} 1-\mathrm{C} 6$ & $107.4(12)$ & $\mathrm{C} 1-\mathrm{C} 7-\mathrm{N} 1$ & $114.9(9)$ \\
\hline $\mathrm{C} 2-\mathrm{C} 1-\mathrm{C} 7$ & $111.0(11)$ & $\mathrm{C} 8-\mathrm{C} 7-\mathrm{H} 7$ & 106.3 \\
\hline $\mathrm{C} 6-\mathrm{C} 1-\mathrm{C} 7$ & $115.2(11)$ & $\mathrm{C} 1-\mathrm{C} 7-\mathrm{H} 7$ & 106.3 \\
\hline $\mathrm{C} 2-\mathrm{C} 1-\mathrm{H} 1$ & 107.7 & $\mathrm{~N} 1-\mathrm{C} 7-\mathrm{H} 7$ & 106.3 \\
\hline $\mathrm{C} 6-\mathrm{C} 1-\mathrm{H} 1$ & 107.7 & $\mathrm{C} 7-\mathrm{C} 8-\mathrm{H} 8 \mathrm{~A}$ & 109.5 \\
\hline $\mathrm{C} 7-\mathrm{C} 1-\mathrm{H} 1$ & 107.7 & $\mathrm{C} 7-\mathrm{C} 8-\mathrm{H} 8 \mathrm{~B}$ & 109.5 \\
\hline $\mathrm{C} 1-\mathrm{C} 2-\mathrm{C} 3$ & $108.4(11)$ & $\mathrm{H} 8 \mathrm{~A}-\mathrm{C} 8-\mathrm{H} 8 \mathrm{~B}$ & 109.5 \\
\hline $\mathrm{C} 1-\mathrm{C} 2-\mathrm{H} 2 \mathrm{~A}$ & 110 & $\mathrm{C} 7-\mathrm{C} 8-\mathrm{H} 8 \mathrm{C}$ & 109.5 \\
\hline $\mathrm{C} 3-\mathrm{C} 2-\mathrm{H} 2 \mathrm{~A}$ & 110 & $\mathrm{H} 8 \mathrm{~A}-\mathrm{C} 8-\mathrm{H} 8 \mathrm{C}$ & 109.5 \\
\hline $\mathrm{C} 1-\mathrm{C} 2-\mathrm{H} 2 \mathrm{~B}$ & 110 & $\mathrm{H} 8 \mathrm{~B}-\mathrm{C} 8-\mathrm{H} 8 \mathrm{C}$ & 109.5 \\
\hline $\mathrm{C} 3-\mathrm{C} 2-\mathrm{H} 2 \mathrm{~B}$ & 110 & $\mathrm{C} 7-\mathrm{N} 1-\mathrm{H} 1 \mathrm{~A}$ & 109.5 \\
\hline $\mathrm{H} 2 \mathrm{~A}-\mathrm{C} 2-\mathrm{H} 2 \mathrm{~B}$ & 108.4 & $\mathrm{C} 7-\mathrm{N} 1-\mathrm{H} 1 \mathrm{~B}$ & 109.5 \\
\hline $\mathrm{C} 4-\mathrm{C} 3-\mathrm{C} 2$ & $107.9(13)$ & $\mathrm{H} 1 \mathrm{~A}-\mathrm{N} 1-\mathrm{H} 1 \mathrm{~B}$ & 109.5 \\
\hline $\mathrm{C} 4-\mathrm{C} 3-\mathrm{H} 3 \mathrm{~A}$ & 110.1 & $\mathrm{C} 7-\mathrm{N} 1-\mathrm{H} 1 \mathrm{C}$ & 109.5 \\
\hline $\mathrm{C} 2-\mathrm{C} 3-\mathrm{H} 3 \mathrm{~A}$ & 110.1 & $\mathrm{H} 1 \mathrm{~A}-\mathrm{N} 1-\mathrm{H} 1 \mathrm{C}$ & 109.5 \\
\hline $\mathrm{C} 4-\mathrm{C} 3-\mathrm{H} 3 \mathrm{~B}$ & 110.1 & $\mathrm{H} 1 \mathrm{~B}-\mathrm{N} 1-\mathrm{H} 1 \mathrm{C}$ & 109.5 \\
\hline $\mathrm{C} 2-\mathrm{C} 3-\mathrm{H} 3 \mathrm{~B}$ & 110.1 & $\mathrm{C} 10-\mathrm{C} 9-\mathrm{C} 14$ & $122.0(10)$ \\
\hline $\mathrm{H} 3 \mathrm{~A}-\mathrm{C} 3-\mathrm{H} 3 \mathrm{~B}$ & 108.4 & $\mathrm{C} 10-\mathrm{C} 9-\mathrm{C} 15$ & $121.8(9)$ \\
\hline $\mathrm{C} 5-\mathrm{C} 4-\mathrm{C} 3$ & $110.3(13)$ & $\mathrm{C} 14-\mathrm{C} 9-\mathrm{C} 15$ & $116.1(9)$ \\
\hline $\mathrm{C} 5-\mathrm{C} 4-\mathrm{H} 4 \mathrm{~A}$ & 109.6 & $\mathrm{C} 9-\mathrm{C} 10-\mathrm{C} 11$ & $122.3(11)$ \\
\hline $\mathrm{C} 3-\mathrm{C} 4-\mathrm{H} 4 \mathrm{~A}$ & 109.6 & $\mathrm{C} 9-\mathrm{C} 10-\mathrm{H} 10$ & 118.8 \\
\hline $\mathrm{C} 5-\mathrm{C} 4-\mathrm{H} 4 \mathrm{~B}$ & 109.6 & $\mathrm{C} 11-\mathrm{C} 10-\mathrm{H} 10$ & 118.8 \\
\hline $\mathrm{C} 3-\mathrm{C} 4-\mathrm{H} 4 \mathrm{~B}$ & 109.6 & $\mathrm{C} 10-\mathrm{C} 11-\mathrm{C} 12$ & $115.2(11)$ \\
\hline $\mathrm{H} 4 \mathrm{~A}-\mathrm{C} 4-\mathrm{H} 4 \mathrm{~B}$ & 108.1 & $\mathrm{C} 10-\mathrm{C} 11-\mathrm{H} 11$ & 122.4 \\
\hline
\end{tabular}




\begin{tabular}{|c|c|c|c|}
\hline $\mathrm{C} 4-\mathrm{C} 5-\mathrm{C} 6$ & $104.8(15)$ & $\mathrm{C} 12-\mathrm{C} 11-\mathrm{H} 11$ & 122.4 \\
\hline $\mathrm{C} 4-\mathrm{C} 5-\mathrm{H} 5 \mathrm{~A}$ & 110.8 & $\mathrm{C} 13-\mathrm{C} 12-\mathrm{C} 11$ & $123.8(10)$ \\
\hline $\mathrm{C} 6-\mathrm{C} 5-\mathrm{H} 5 \mathrm{~A}$ & 110.8 & $\mathrm{C} 13-\mathrm{C} 12-\mathrm{Br} 1$ & $119.8(9)$ \\
\hline $\mathrm{C} 4-\mathrm{C} 5-\mathrm{H} 5 \mathrm{~B}$ & 110.8 & $\mathrm{C} 11-\mathrm{C} 12-\mathrm{Br} 1$ & $116.5(9)$ \\
\hline $\mathrm{C} 6-\mathrm{C} 5-\mathrm{H} 5 \mathrm{~B}$ & 110.8 & $\mathrm{C} 12-\mathrm{C} 13-\mathrm{C} 14$ & $121.9(10)$ \\
\hline $\mathrm{H} 5 \mathrm{~A}-\mathrm{C} 5-\mathrm{H} 5 \mathrm{~B}$ & 108.9 & $\mathrm{C} 12-\mathrm{C} 13-\mathrm{H} 13$ & 119 \\
\hline $\mathrm{C} 5-\mathrm{C} 6-\mathrm{C} 1$ & $115.8(13)$ & $\mathrm{C} 14-\mathrm{C} 13-\mathrm{H} 13$ & 119 \\
\hline $\mathrm{C} 5-\mathrm{C} 6-\mathrm{H} 6 \mathrm{~A}$ & 108.3 & $\mathrm{C} 13-\mathrm{C} 14-\mathrm{C} 9$ & $114.7(11)$ \\
\hline $\mathrm{C} 1-\mathrm{C} 6-\mathrm{H} 6 \mathrm{~A}$ & 108.3 & $\mathrm{C} 13-\mathrm{C} 14-\mathrm{H} 14$ & 122.6 \\
\hline $\mathrm{C} 5-\mathrm{C} 6-\mathrm{H} 6 \mathrm{~B}$ & 108.3 & $\mathrm{C} 9-\mathrm{C} 14-\mathrm{H} 14$ & 122.6 \\
\hline $\mathrm{C} 1-\mathrm{C} 6-\mathrm{H} 6 \mathrm{~B}$ & 108.3 & $\mathrm{O} 2-\mathrm{C} 15-\mathrm{O} 1$ & $127.7(9)$ \\
\hline $\mathrm{H} 6 \mathrm{~A}-\mathrm{C} 6-\mathrm{H} 6 \mathrm{~B}$ & 107.4 & $\mathrm{O} 2-\mathrm{C} 15-\mathrm{C} 9$ & $118.2(8)$ \\
\hline $\mathrm{C} 8-\mathrm{C} 7-\mathrm{C} 1$ & $114.4(11)$ & $\mathrm{O} 1-\mathrm{C} 15-\mathrm{C} 9$ & $114.0(8)$ \\
\hline $\mathrm{C} 8-\mathrm{C} 7-\mathrm{N} 1$ & $108.0(11)$ & & \\
\hline $\mathrm{C} 6-\mathrm{C} 1-\mathrm{C} 2-\mathrm{C} 3$ & $56.2(16)$ & $\mathrm{C} 15-\mathrm{C} 9-\mathrm{C} 10-\mathrm{C} 11$ & $173.2(10)$ \\
\hline $\mathrm{C} 7-\mathrm{C} 1-\mathrm{C} 2-\mathrm{C} 3$ & $-177.1(13)$ & $\mathrm{C} 9-\mathrm{C} 10-\mathrm{C} 11-\mathrm{C} 12$ & $2.1(16)$ \\
\hline $\mathrm{C} 1-\mathrm{C} 2-\mathrm{C} 3-\mathrm{C} 4$ & $-60.0(18)$ & $\mathrm{C} 10-\mathrm{C} 11-\mathrm{C} 12-\mathrm{C} 13$ & $-1.5(16)$ \\
\hline $\mathrm{C} 2-\mathrm{C} 3-\mathrm{C} 4-\mathrm{C} 5$ & $63.5(19)$ & $\mathrm{C} 10-\mathrm{C} 11-\mathrm{C} 12-\mathrm{Br} 1$ & $179.8(8)$ \\
\hline $\mathrm{C} 3-\mathrm{C} 4-\mathrm{C} 5-\mathrm{C} 6$ & $-62.2(18)$ & $\mathrm{C} 11-\mathrm{C} 12-\mathrm{C} 13-\mathrm{C} 14$ & $1.4(17)$ \\
\hline $\mathrm{C} 4-\mathrm{C} 5-\mathrm{C} 6-\mathrm{C} 1$ & $62(2)$ & $\mathrm{Br} 1-\mathrm{C} 12-\mathrm{C} 13-\mathrm{C} 14$ & $-179.9(8)$ \\
\hline $\mathrm{C} 2-\mathrm{C} 1-\mathrm{C} 6-\mathrm{C} 5$ & $-61.5(19)$ & $\mathrm{C} 12-\mathrm{C} 13-\mathrm{C} 14-\mathrm{C} 9$ & $-1.8(15)$ \\
\hline $\mathrm{C} 7-\mathrm{C} 1-\mathrm{C} 6-\mathrm{C} 5$ & $174.3(14)$ & $\mathrm{C} 10-\mathrm{C} 9-\mathrm{C} 14-\mathrm{C} 13$ & $2.5(14)$ \\
\hline $\mathrm{C} 2-\mathrm{C} 1-\mathrm{C} 7-\mathrm{C} 8$ & $67.0(16)$ & $\mathrm{C} 15-\mathrm{C} 9-\mathrm{C} 14-\mathrm{C} 13$ & $-173.7(9)$ \\
\hline $\mathrm{C} 6-\mathrm{C} 1-\mathrm{C} 7-\mathrm{C} 8$ & $-170.7(15)$ & $\mathrm{C} 10-\mathrm{C} 9-\mathrm{C} 15-\mathrm{O} 2$ & $155.7(11)$ \\
\hline $\mathrm{C} 2-\mathrm{C} 1-\mathrm{C} 7-\mathrm{N} 1$ & $-58.7(15)$ & $\mathrm{C} 14-\mathrm{C} 9-\mathrm{C} 15-\mathrm{O} 2$ & $-28.1(14)$ \\
\hline $\mathrm{C} 6-\mathrm{C} 1-\mathrm{C} 7-\mathrm{N} 1$ & $63.5(17)$ & $\mathrm{C} 10-\mathrm{C} 9-\mathrm{C} 15-\mathrm{O} 1$ & $-20.1(14)$ \\
\hline $\mathrm{C} 14-\mathrm{C} 9-\mathrm{C} 10-\mathrm{C} 11$ & $-2.8(17)$ & $\mathrm{C} 14-\mathrm{C} 9-\mathrm{C} 15-\mathrm{O} 1$ & $156.1(9)$ \\
\hline
\end{tabular}

Hydrogen-bond geometry $\left(\AA,{ }^{\circ}\right)$

\begin{tabular}{lllll}
\hline$D-\mathrm{H} \cdots A$ & $D-\mathrm{H}$ & $\mathrm{H} \cdots A$ & $D \cdots A$ & $D-\mathrm{H} \cdots A$ \\
\hline $\mathrm{N} 1-\mathrm{H} 1 A \cdots \mathrm{O} 1$ & 0.91 & 1.99 & $2.781(12)$ & 144 \\
$\mathrm{~N} 1-\mathrm{H} 1 B \cdots \mathrm{O} 2^{\mathrm{i}}$ & 0.91 & 2.06 & $2.870(12)$ & 148 \\
$\mathrm{~N} 1-\mathrm{H} 1 C \cdots \mathrm{O} 1^{\mathrm{ii}}$ & 0.91 & 1.89 & $2.718(10)$ & 150 \\
\hline
\end{tabular}

Symmetry codes: (i) $x+1, y, z$; (ii) $x+1 / 2,-y+1 / 2,-z+1$. 